NASA Contractor Report 3952

$$
\begin{aligned}
& \text { NASA-CR-3952 } \\
& 19860012832
\end{aligned}
$$

\title{
Coherent Large-Scale Structures in High Reynolds Number Supersonic Jets
}

J. Lepicovsky, K. K. Ahuja, W. H. Brown, and R. H. Burrin

CONTRACT NAS1-17442

DECEMBER 1985

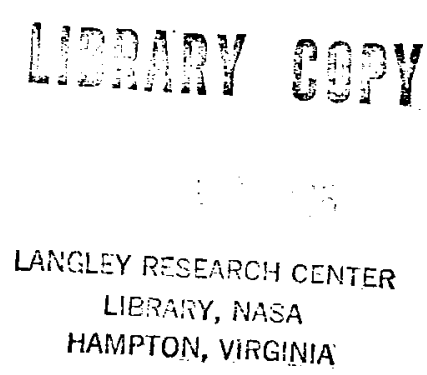


NASA Contractor Report 3952

\section{Coherent Large-Scale Structures in High Reynolds Number Supersonic Jets}

J. Lepicovsky, K. K. Ahuja, W. H. Brown, and R. H. Burrin

Lockbeed-Georgia Company

Marietta, Georgia

Prepared for

Langley Research Center

under Contract NAS1-17442

\section{N/SA}

National Aeronautics

and Space Administration

Scientific and Technical Information Branch 
This Page Intentionally Left Blank 


\section{FOREWORD}

This report was prepared by Lockheed-Georgia Company, Marietta, for NASALangley Research Center, Hampton, Virginia, under Contract NAS1-17442, entitled, "A Study of Coherent Large-Scale Structures in High Reynolds Number Supersonic Jets."

Dr. J. Seiner was the Project Manager for the NASA Langley Research Center. Lockheed's Program Manager was Dr. K. K. Ahuja.

Continuous support of Mr. Alan Proffitt, Mr. R. H. Huie, Mr. J. F. Songer, and Dr. W. Bell in troubleshooting the LV electronics and softwarerelated problems is gratefully acknowledged. 
TABLE OF CONTENTS

Page

SUMMARY. . . . . . . . . . . . . . . . . . . . . 1

1.0 INTRODUCTION . . . . . . . . . ........... 3

1.1 Basis of this study. . . . . . . . . . . . . . 3

1.2 objective................... . . 4

1.3 Report Out1ine ................... 4

2.0 TEST FACILITIES AND EXPERIMENTAL PROCEDURES. . . . . . . . . . . 5

2.1 Air Supply and the Flow Plenum . . . . . . . . . . . . . 5

2.2 The Test Nozzle...................... 5

2.3 Acoustic Excitation Source . . . . . . . . . . . 8

2.4 Flow Visualization Optics. . . . . . . . . . . . . . 8

2.5 Laser Velocimeter System . . . . . . . . . . . . . . 10

2.6 Conditional Sampling Procedure . . . . . . . . . . . 16

2.7 Test Conditions. . . . . . . . . . . . . . . . 20

2.7.1 Task I - Flow Visualization of Large-Scale

Structures ................. 20

2.7.2 Task II - LV Quantitative Measurements of

Large-Scale structures . . . . . . . . 20

3.0 FLOW VISUALIZATION RESULTS . . . . . . . . . . . . . . . 23

3.1 Excitation Level Effects . . . . . . . . . . . . . . . 23

3.2 Excitation Frequency Effects . . . . . . . . . . . . . 26

3.3 Time Delay Experiments . . . . . . . . . . . . . . 33

3.4 Initial Conclusions ................. 45

$4.0 \mathrm{LV}$ QUANTITATIVE MEASUREMENTS ................. . . 47

4.1 Jet-Noise Spectra . . . . . . . . . . . . . . . 47

4.2 Mean Flow Measurements . . . . . . . . . . . . . . . 47

4.3 Conditionally Sampled LV Measurements............. 63

4.4 Comparison with Other Existing Measurements. . . . . . . . 67

4.4.1 Mean Flow Characteristics . . . . . . . . . 67

4.4.2 Turbulence Intensity. ............... 69

4.4.3 Instability Strouhal Number ........... . 69

4.4.4 Phase Velocity ................ 69

4.5 Conclusions. . . . . . . . . . . . . . . . . . 74

5.0 PROBLEMS ENCOUNTERED IN THIS PROJECT, THEIR SOLUTIONS, AND LESSONS

LEARNED THEREFROM . . . . . . . . . . . . . . . . . . . . . . 77

5.1 Problems with Flow Visualization . . . . . . . . . . . 77

5.2 Problems with LV Measurements. . ........... . . 84

5.2.1 Signal-to-Noise Ratio Problem . . . . . . . . . 84

5.2.2 Hardware-Related Low Data Rate Problem. . . . . . . 86

5.2.3 LV Frequency Response.............. . 87

5.3 Concluding Remarks.................. 88 
References........................ . . 91

Appendix A - Flow Visualization for Nozzle Selection . . . . . . . 95

Appendix B - List of Symbols . . . . . . . . . . . . . . . 99 


\section{SUMMARY}

The major objective of this program was to investigate the existence and behavior of large-scale turbulent structures in a high Reynolds number supersonic jet. To achieve this objective, a systematic set of flow visualization experiments and quantitative flow measurements of a supersonic, fully expanded jet of $M_{j}=1.4$ were made in the free jet research facility of the Lockheed-Georgia Company. To lift up the large-scale structure from within the random turbulence in the jet shear layer, the jet was excited by upstream tone. However, the level of excitation was kept as low as possible not to turn the program into a study of "flow modification by sound."

Advanced experimental methods were used to perform the planned experiments. Flow pictures were acquired using a unique laser schlieren system enabling photographic ensemble averaging, revealing thus the existence of the large-scale turbulent structure in the jet shear layer. Lockheed's laser velocimeter system with conditional sampling was used for flowfield measurements to quantify the qualitative results obtained under the flow visualization task.

Several serious technical problems were encountered during the course of this project. A detailed analysis of these problems and a review of the lessons learned are also presented.

The salient conclusions derived from the results for the high Reynolds number, fully expanded supersonic jet of $M_{j}=1.4$ can be listed as follows:

1. The large-scale structures do exist in high Reynolds number supersonic jets and they prevail even beyond the potential core of the jet.

2. Quantitatively, the large-scale structures excited under the present test conditions were found to be rather weak, and their peak amplitudes were only about $1 \%$ of the mean jet exit velocity.

3. The most preferential Strouhal number for these structures is in the vicinity of 0.4 . This agrees very well with the reported findings for low Reynolds number supersonic jets.

4. The dependence of the large-scale structure phase velocity on the excitation Strouhal number, under the present test conditions, shows the same trend as found for subsonic tone-excited jets. 


\subsection{INTRODUCTION}

1.1 Basis of this Study

The description of the large-scale motions present in free turbulent flows dates back, at least, to Townsend (1956, Ref.1.1). Crow and Champagne (Ref. 1.2) and Brown and Roshko (Ref. 1.3, 1.4) were among the first, however, to highlight the importance of the large-scale turbulence in jet mixing layers.

Since then, the "orderly" structure in the turbulence of free shear flows is receiving the attention of numerous researchers. A number of review articles on large turbulence structures have since appeared in the literature; e.g., Roshko (Ref. 1.5), Cantwell (1.6), Hussain (Ref. 1.7), and Ho and Huerre (1.8). The consensus of most investigators appears to be that these structures are characteristic features of shear flows.

In the area of jet noise, it has been suggested by a number of investigators (Refs. $1.9-1.14$ ) that the dominant part of turbulent mixing noise of supersonic jets is generated directly by the large turbulence structures or instability waves of the jet flow. When this suggestion was first made, it was qualitative and based primarily on theoretical reasonings alone. Motivated by this theoretical proposal, McLaughlin, Morrison and Troutt (Refs. 1.15, 1.16) showed experimentally that instability waves were the primary noise generators for perfectly expanded supersonic jets. The experiments were difficult to carry out. In order to avoid many of the experimental problems inherent in high Reynolds number flows, they used a low density (thus low Reynolds number) supersonic jet facility. They found that, for low Reynolds number supersonic jets, the large-scale structures were in the form of instability waves. These waves were coherent over an axial distance of many jet diameters. In addition, these large instability waves generated an intense acoustic field, which extended from the jet flow all the way to the boundary of the anechoic jet flow facility. To assess the effects of Reynolds number, Troutt and McLaughlin (Ref.1.16) repeated their earlier experiment at moderately high Reynolds numbers. They found that the unsteady motion of the supersonic jet was dominated by a band of large-scale instability waves. The characteristics of these instability waves were similar to those of the low Reynolds number experiments. In addition, at these moderately high Reynolds numbers, the dominant part of the acoustic field was generated by the large-scale instability waves. Troutt and McLaughlin (Ref. 1.16) and Seiner, McLaughlin, and Liu (Ref. 1.17) compared the near field noise contours and the far field noise directivity and spectral characteristics of these jets with those of high Reynolds number jets and found remarkable resemblence.

Thus, there are clear implications that large-scale coherent-like structures should dominate the initial flow field of high Reynolds number supersonic jet. At this point, however, it is not clear to what extent, if any, these coherent structures influence the jet noise generation process of high Reynolds number supersonic jets of technological interest. Such knowledge is urgently needed for verification of new theoretical models that specifically incorporate these structures. 


\subsection{Objective}

The overall goal of this program was to obtain a detailed understanding of the behavior of large-scale structures in a high Reynolds number supersonic jet where, based on exit conditions, the Reynolds number Re exceeds one million.

The specific objective was to determine, through a basic and we11controlled experiment, if large-scale coherent-like structures exist in high Reynolds number shock-free supersonic jets and, if so, determine their characteristics as they relate to flow development and jet noise radiation.

This program was particularly focused at studying the time-dependent structure associated with the flow from an unheated convergent-divergent nozzle operating shock-free at fully-expanded Mach number of 1.37. The exit diameter of the nozzle was $50.8 \mathrm{~mm}$ ( 2 in.), and the corresponding Reynolds number, Re, was $2.35 \times 10^{6}$ for the unheated jet and $1.72 \times 10^{6}$ for the isothermal jet. The flow structure of the first 20 jet diameters was examined. In order to facilitate the study of the large-scale structure and determine any coherence, a discrete tone acoustic excitation method along with a means of phase averaging the measured flow structure (described later) was incorporated.

\subsection{Report Outline}

The details of the test facilities and the experimental procedures are given in the Section 2.0 .

The research consisted of the following two technical tasks:

Task I - Flow Visualization of Large-Scale Structures

Task II - LV Quantitative Measurements of Large-Scale Structures

Although each of the above tasks are independent, the research was performed chronologically in the above order. These two tasks are described separately in Sections 3.0 and 4.0 , respectively.

Several serious problems were encountered during the course of this work. Because of their importance to similar work by other researchers, a separate section (Section 5.0) is devoted to providing their description and an overview of the steps taken to overcome them. Where possible, recommendations for future experimental work are also included. 


\subsection{TEST FACILITIES AND EXPERIMENTAL PRDCEDURES}

A11 experiments described in this report were conducted in Lockheed's jet-flow facility. This is the same facility that has been used in the past for various jet-related turbulence measurements for both single and coannular jets. A detailed description of this facility is given in Reference 2.1 and the salient features are summarized below.

\subsection{Air Supply and the Flow Plenum}

The jet-flow facility used in this program is designed to produce paralle1, low-turbulence, coaxial flows, or single-stream flow with provision for simulating flight effects with a large secondary nozzle. Only the primary or inner flow was used for the present experiments. A cross-sectional view of the facility is shown in Figure 2.1. The primary flow enters through a 256mn-dia plenum, followed by an initial contraction to 102-mm-dia source-section duct. The test nozzle used in this program is 50.8-mm-dia C-D nozzle, which is attached directly to the source-section duct. The plenum to nozzle area contraction ratio is 25 .

The jet flow in this facility can be heated to temperatures reaching $1000 \mathrm{~K}$ at pressure ratios exceeding 4 . The flow is heated by a through-flow propane burner. Both pressure and temperature conditions can be controlled either by a computer or manually.

\subsection{The Test Nozzle}

As described in Appendix A, three separate convergent-divergent nozzles were available for testing. Each nozzle had a diameter of $50.8 \mathrm{~mm}$ (2 in.) and a design Mach number of 1.37. After thorough flow visualization, one of the three nozzles was selected for this program. This nozzle is shown in Figure 2.2. This was the same nozzle that had been used at Lockheed for extensive jet noise measurements in the $70 \mathrm{~s}$ (Ref. 2.2).

The design and manufacture of the convergent nozzle is a relatively simple matter, but the convergent-divergent nozzles are particularly difficult in both design and construction. The contour of the divergent section downstream of the throat must be very carefully controlled in order to expand the flow smoothly to the exit plane. The design of the nozzle used here was accomplished employing the well established method of characteristics. The question of boundary layer displacement correction is not so well established and, in the original design of this nozzle, it was decided not to attempt to correct the contours for this effect. This omission has the effect of reducing the area ratio between the exit and the throat, and hence the fullyexpanded Mach number and pressure ratio will also be reduced.

The nozzle was originally manufactured from Inconel stainless steel in order to be compatible with high temperature operation. The nozzle profiles were first drawn out five times actual size using a special computer splining routine. These drawings were then placed on a contour following device, controlling a 'slave' vertical milling machine, with which an actual size thin 


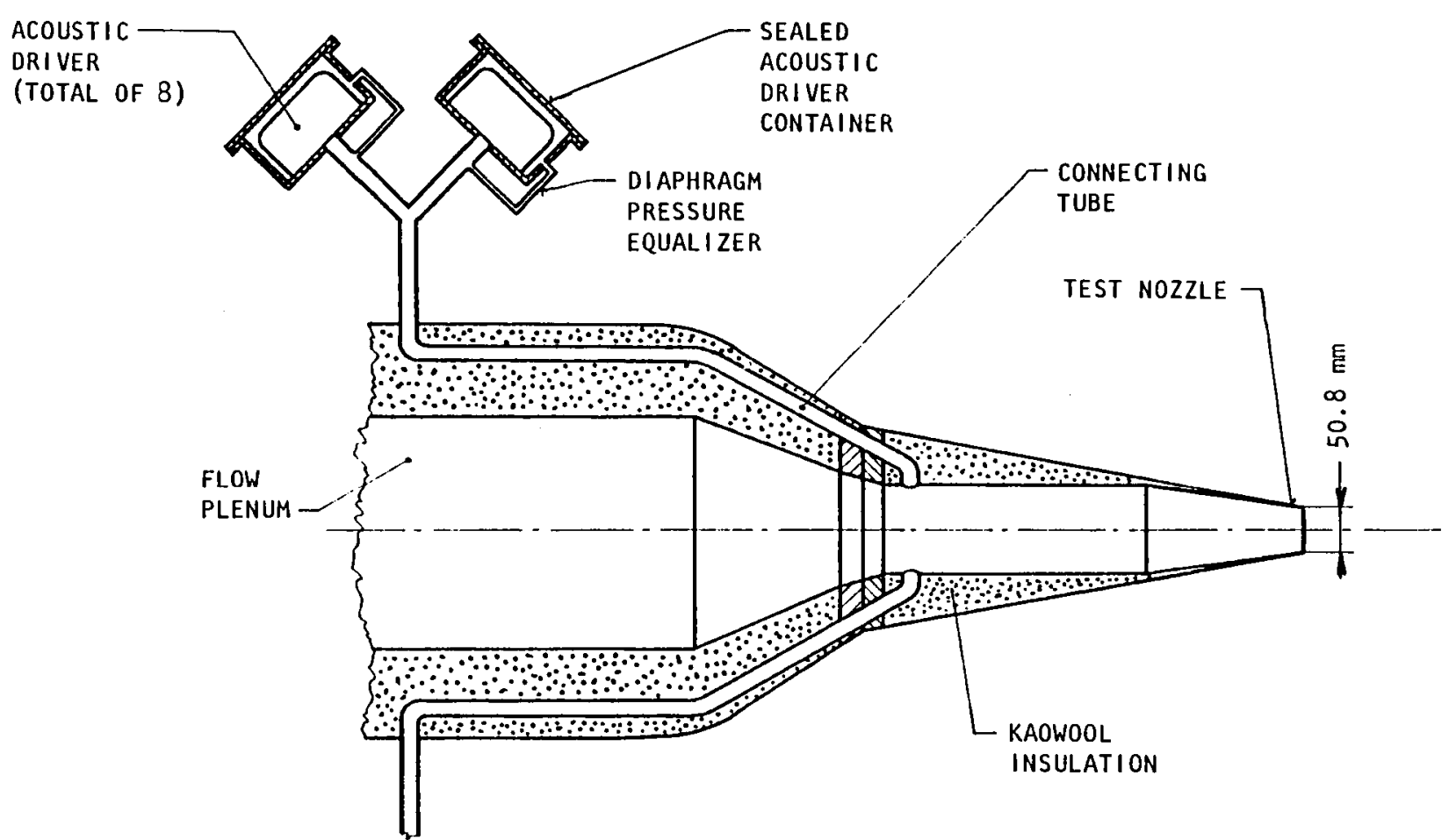

Figure 2.1 Jet flow facility and source section. 


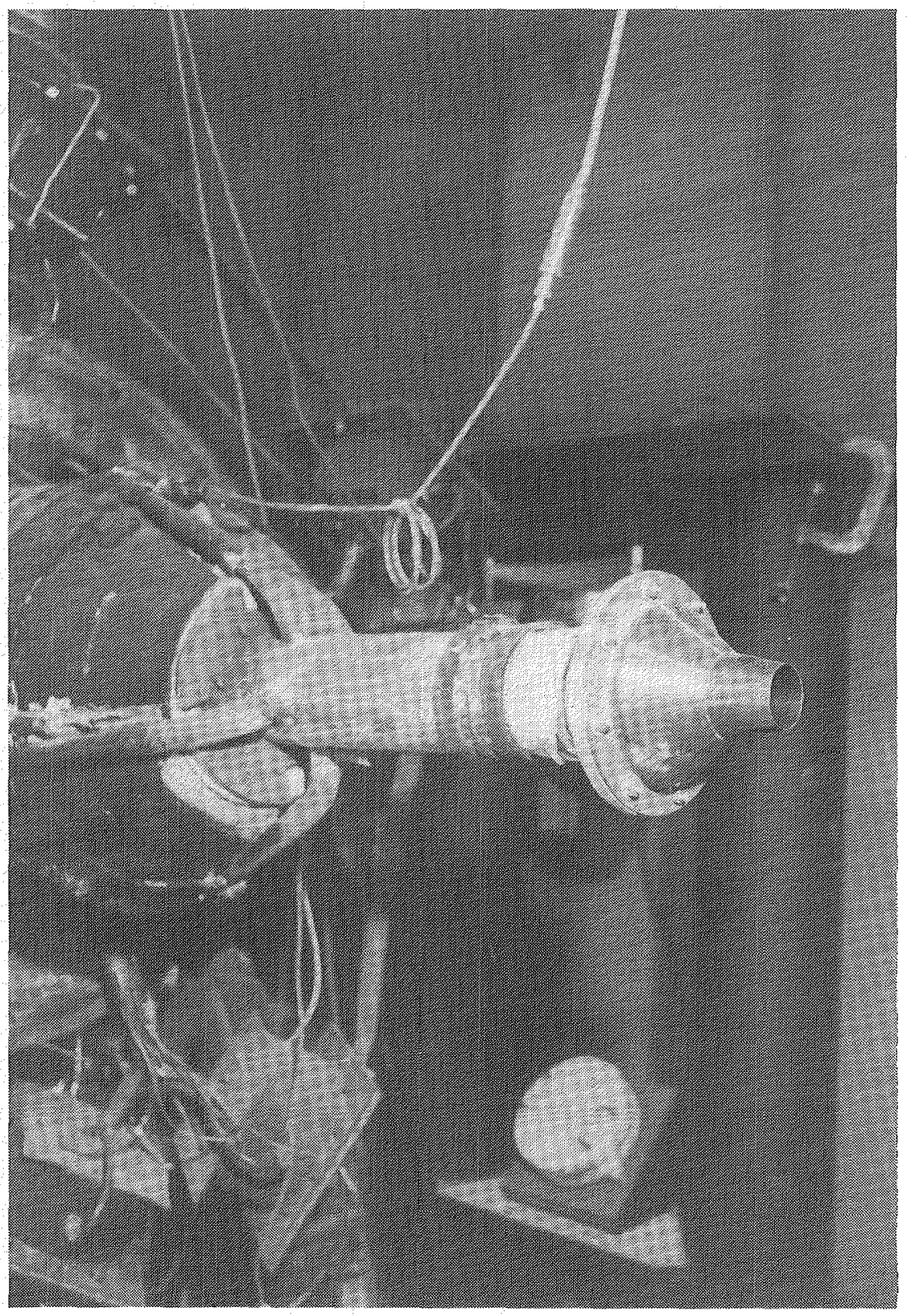

Figure 2.2 Test nozzle. 
p1ate steel template was cut. Thus, any errors in the original drawing were reduced by a factor of 5 . The template was then transferred to a template lathe and the nozzle bored out using a specially constructed stiff boring bar to minimize tool jitter. During the initial boring, the optimum depth and speed of cut to give the best surface finish was determined. The final cut was made on the basis of these findings. Final polishing was done using a very fine grinding paste.

\subsection{Acoustic Excitation Source}

The source section used in this series of experiments is the same source section that was used in Lockheed's earlier studies on acoustically exited jets conducted under a NASA Lewis contract (Ref, 2.1). The source section is depicted in Figure 2.1. It is centered around a 102-mm-dia air supp1y duct, which is connected to a test nozzle. It utilizes eight 100-W ALTEC (model 1290E) acoustic driver units. Each driver is enclosed in a pressure vessel to equalize the pressure across the driver diaphragm. Each driver is fed the same electronic signal which effectively enables each driver to generate sound in phase. The tubes connecting the drivers to the 102-mm-dia air supply duct have provision for cooling air for the high-temperature tests in order to protect the diaphragms.

The sound is funneled to the 102-mm-dia duct through four 25.4-mm-dia tubes with each tube connected to a pair of acoustic drivers through a "Y" connector, as seen in Figure 2.3.

Since it is important that the $25.4-\mathrm{mm}$-dia tubes connecting the driver section to the air supply duct do not produce significant flow disturbances other than the excitation signal itself, the source-duct-nozzle system has been thoroughly calibrated in the past and found to be aerodynamically acceptable.

\subsection{Flow Visualization Optics}

Most shadowgraphs and schlieren photographs, particularly those for axisymetric flows, display a certain degree of confused detail resulting from smal1-scale turbulence in the jet, and from thermal convection in the ambient air. A method of removing these sometimes unwanted details, and thereby of highlighting essential details, is the app1ication of a photographic averaging technique which is an effective method for revealing large-scale coherent structure even in an unexcited jet. The method consists of repeated triggering of a light source and superposition of all the schlieren images on a single photographic film. By this means, the image of the coherent structure associated with the trigger signal is reinforced, and the contribution from random turbulence tends to cancel out.

The above method was used in the present experiments also, but a new and very simple method of synchronizing the source of light was used. A laser beam together with a Bragg cell was the source of light. A description of this new schlieren system can be found in Reference 2.3 . 


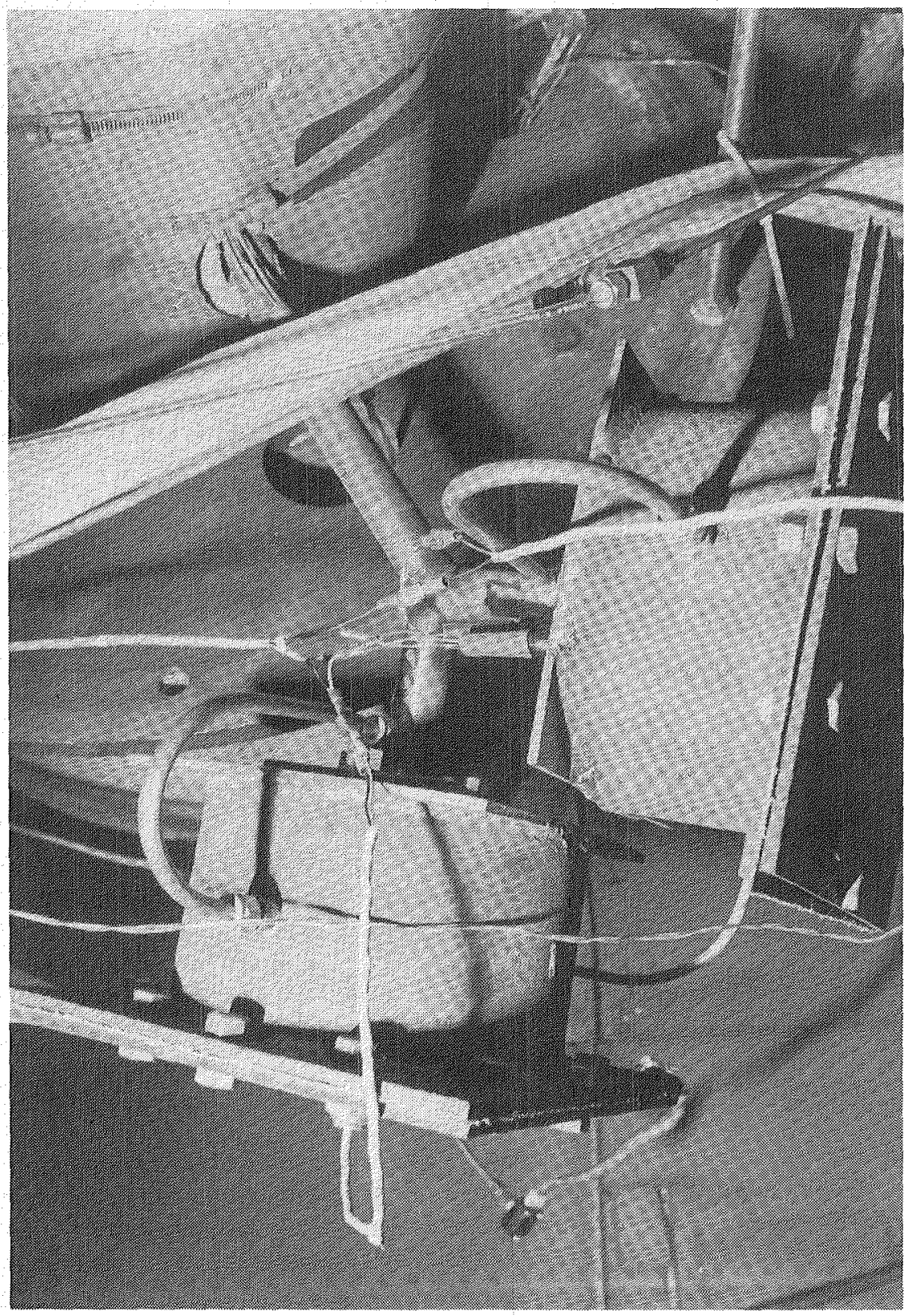

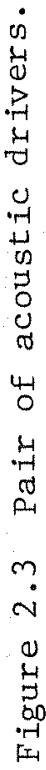


A block diagram of the optical setup is shown in Figure 2.4. It features a Bragg cell that pulses the light beam from a CN Argon laser.

In operation, the Bragg cell acts as a shutter blocking the laser beam. In this application, when the cell is excited at its center frequency, the light beam is deflected through the selecting aperture and aligned with the schlieren optics. A pulsed shutter is obtained by using a diode switch to interrupt the excitation except when modulated by the pulse generator.

Two modes of operation are available with the present arrangement. In the multiple pulse mode, the Bragg cell is continually pulsed by the acoustic signal, and the camera shutter is adjusted to average the light from several pulses to illuminate the large-scale structure. In the single pulse mode, the camera shutter is used to limit exposure to background light (some residual light comes through the selecting aperture) and the shutter strobe opens a latch that allows the next audio trigger to pulse the Bragg cell.

The power level of the laser-light source is adjustable up to several watts, providing excellent film exposure control.

The schlieren system itself was actually a converging-mirror type (Ref. 2.4), wherein the light makes a double-pass through the test section as shown in the lower half of Figure 2.4. The detailed paths of the light beam axe shown in Figure 2.5. The system was a wedge mirror instead of a splitter plate to separate the beams from the source and to the knife edge.

A photographic view of the complete system along with the laser light path is shown in Figure 2.6.

It should be pointed out that to visualize a rather extended axial region of the jet, the whole optical bench could be traversed. This was accomplished by moving the traverse bed " $A$ " and the columnar supports " $B$ " and " $C$ " shown in Figure 2.7, part of which can be seen in Figure 2.6 also. Using a $0.406 \mathrm{~m}$ (16 inch) diameter converging mirror on Support " $A$ ", it enabled the visualization of the jet flow as far downstream as 20 jet diameters in four separate traverses.

\subsection{Laser Velocimeter System}

The mean velocities and the turbulence intensities were measured by Lockheed's laser velocimeter system, which has been used extensively in the past to make both single and coannular jet-flow surveys. A detailed description of this facility is given in Reference 2.5, and the salient features are summarized below.

The LV facility is shown in Figure 2.8 as an artists impression, which illustrates the coannular jet nozzle ducting and the dual laser velocimeter systems.

The laser velocimeter used in this study was an advanced counter type, two-color, four-channel LV developed at Lockheed-Georgia Company. It enables 


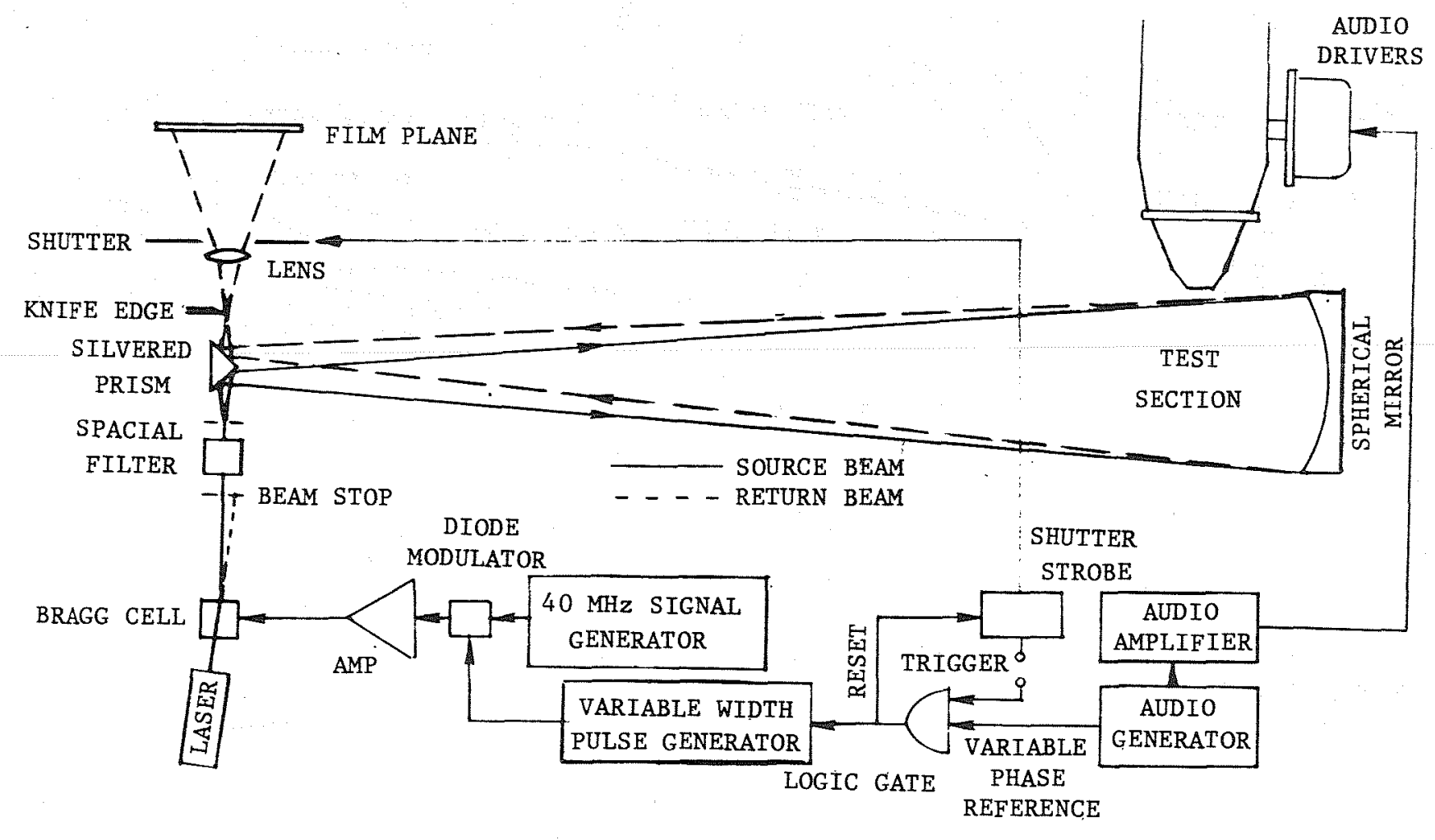

Figure 2.4 Flow visualization setup and the associated electronics. 


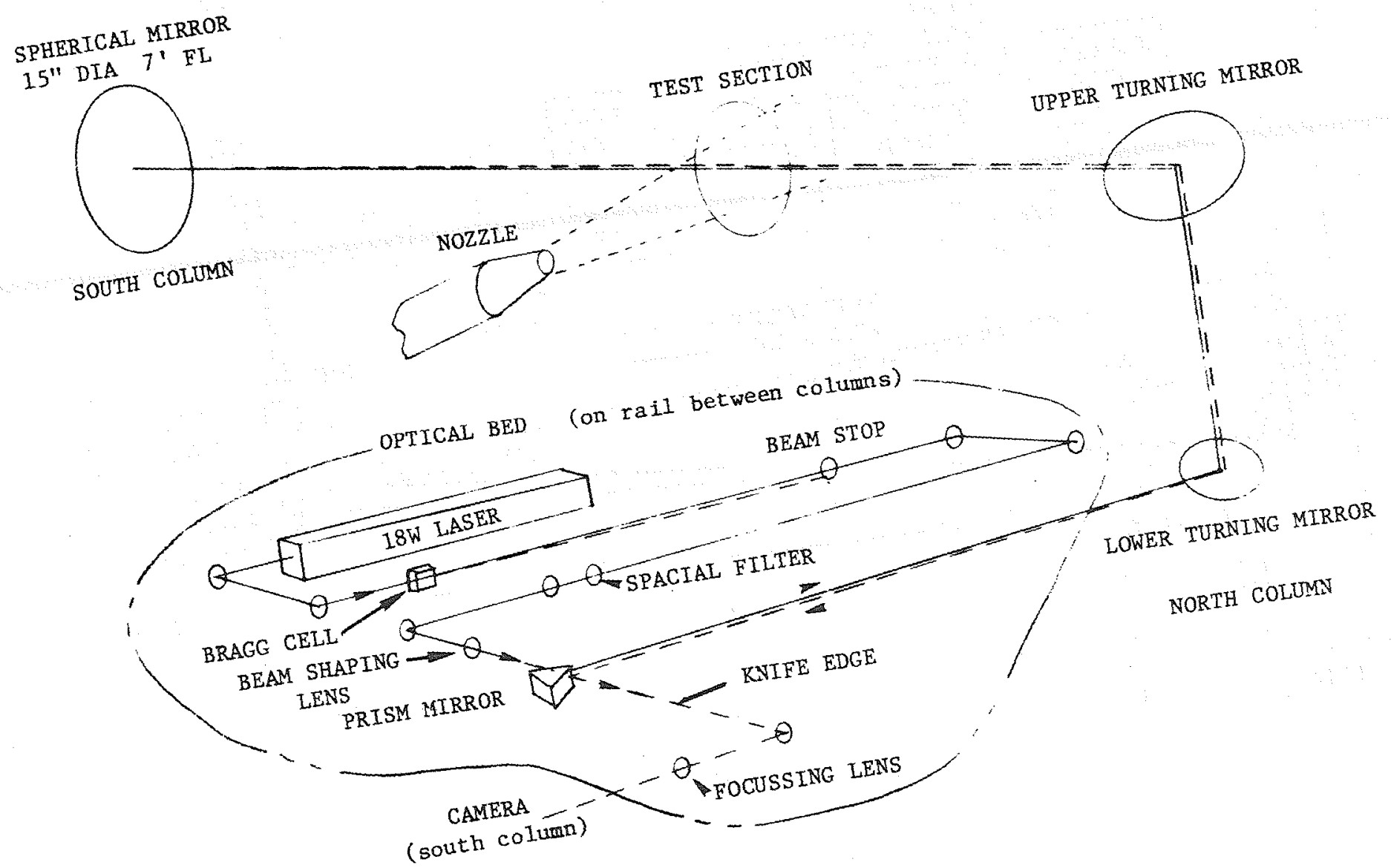

Figure 2.5 Detailed paths of the light beam. 


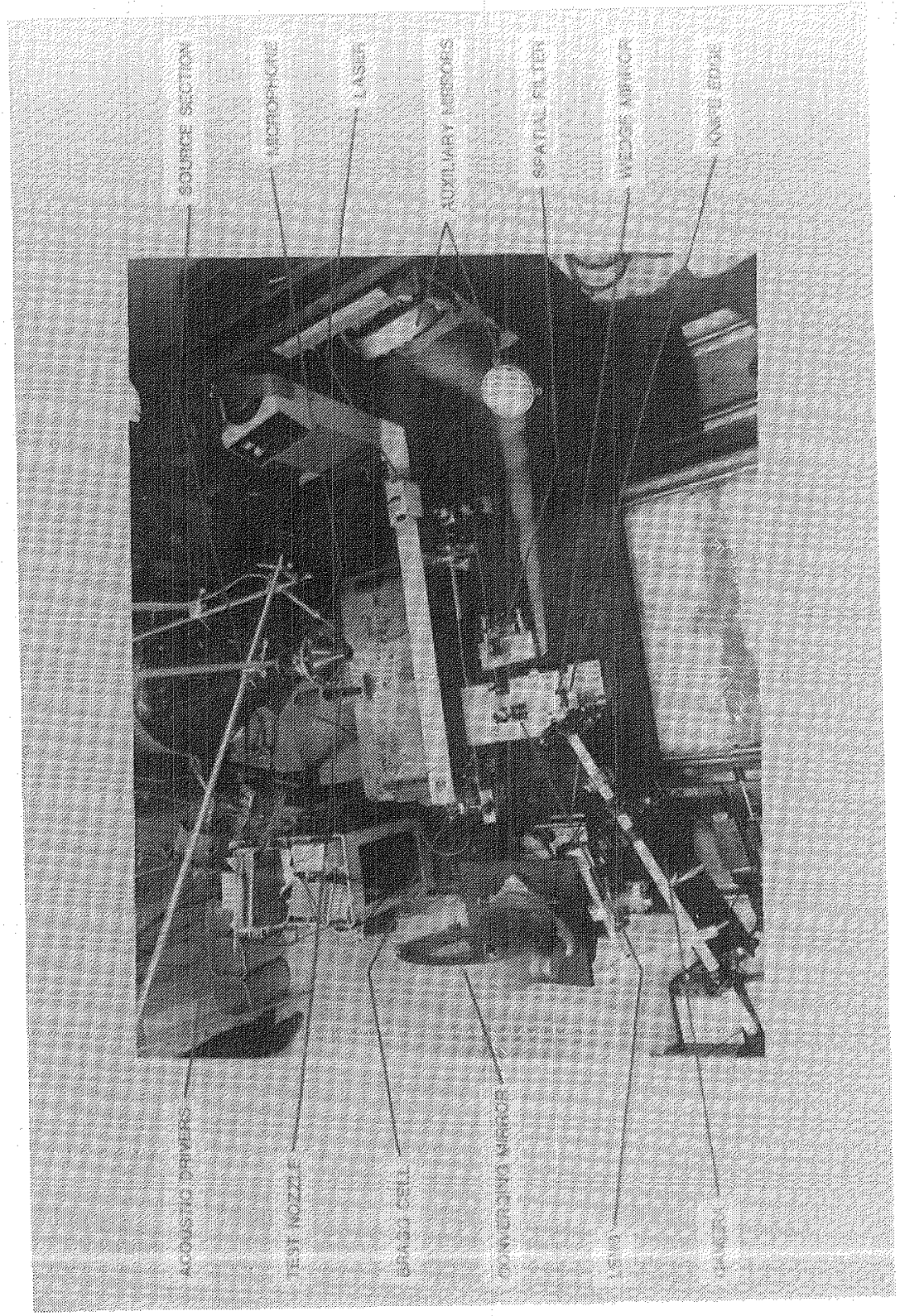

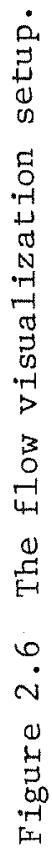




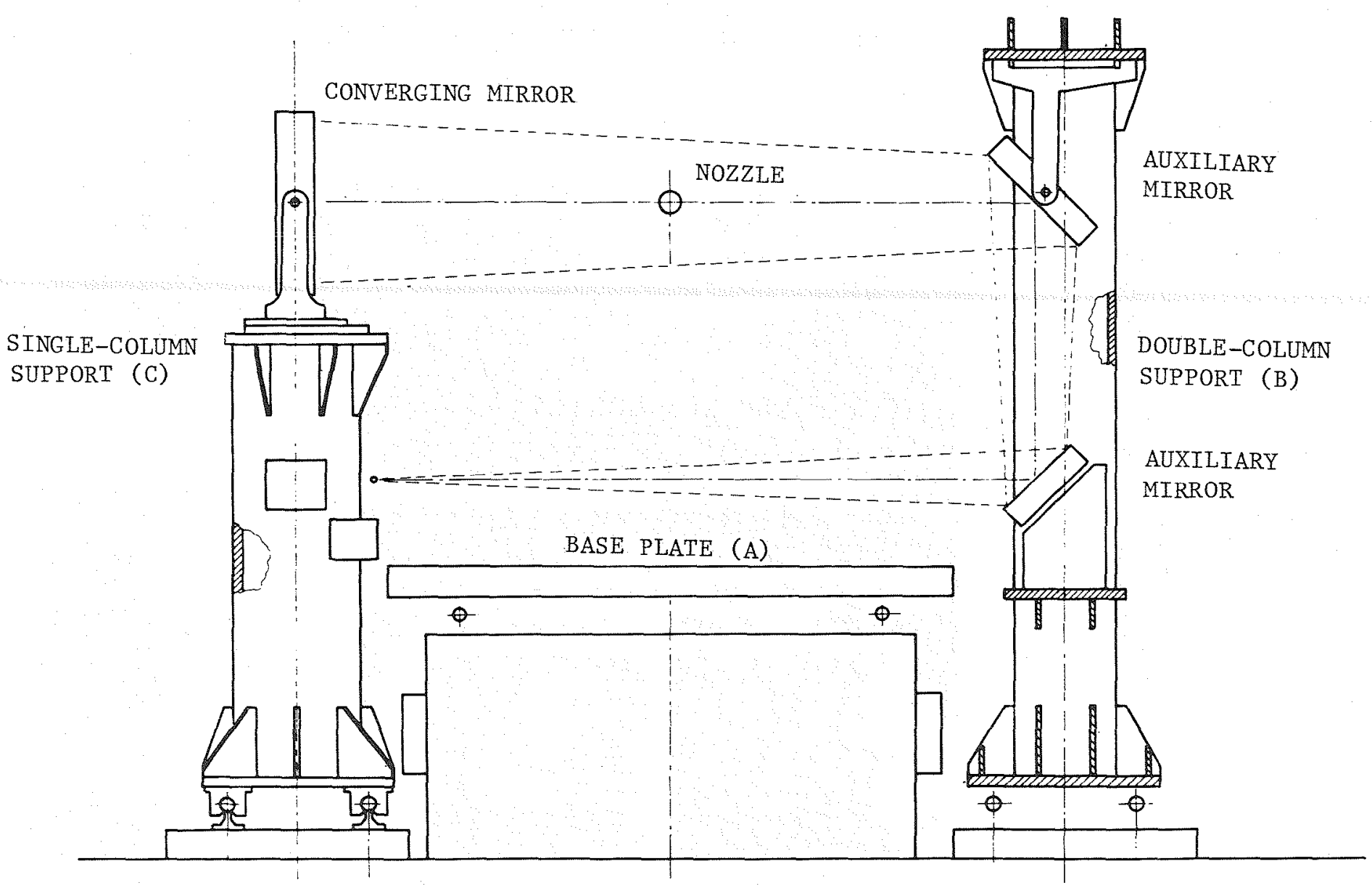

Figure 2.7 Traversable bed and supports used in the flow visualization. 


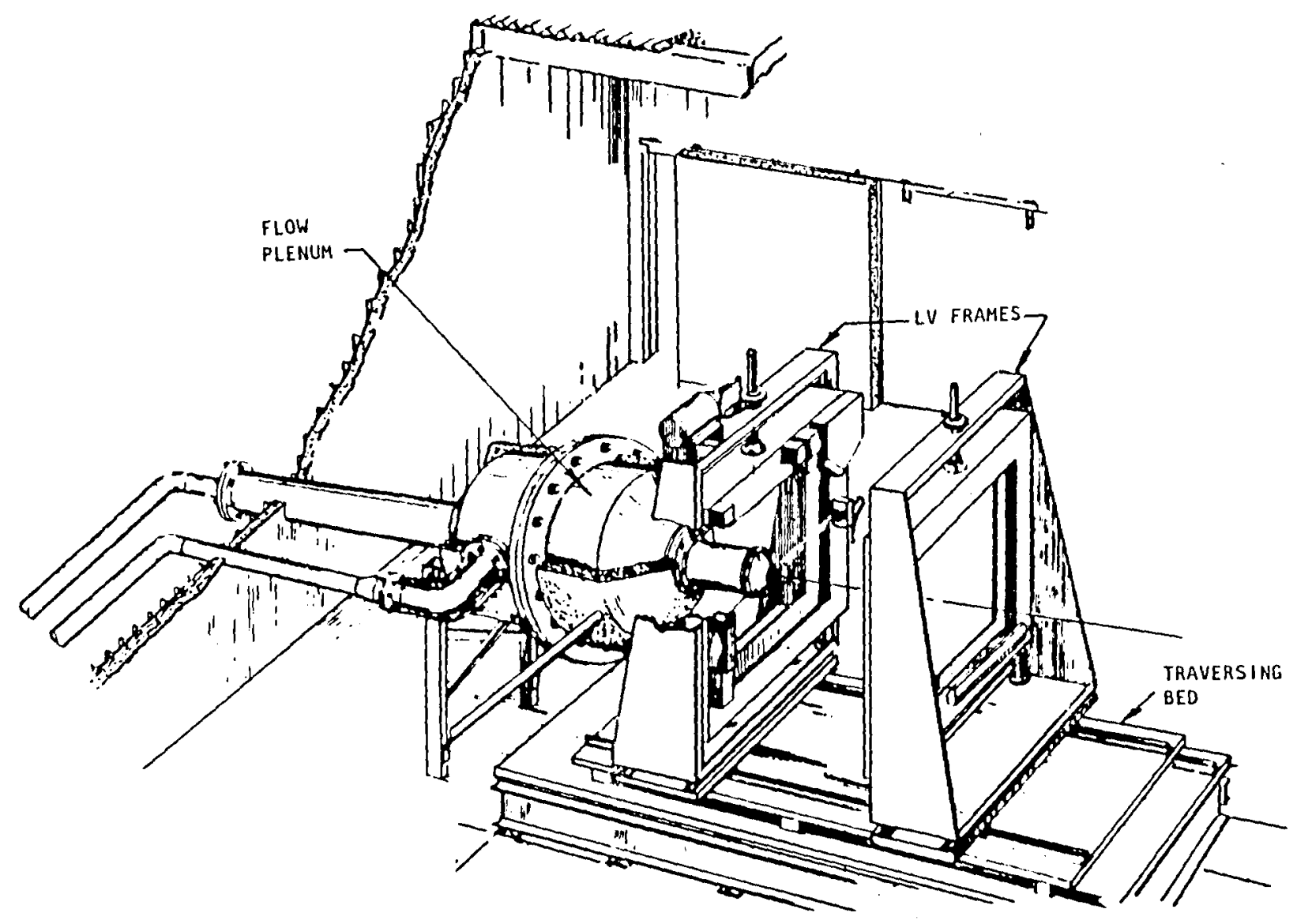

Figure 2.8 Jet turbulence facility with LV traversing bed and frames. 
simultaneous two-component velocity measurement. Each of the velocity components is measured by two separate counters. This unique arrangement practically eliminates the photomultiplier tube noise contribution to the measured turbulence intensity leve1. The LV for this particular measurement was arranged in a forward scatter mode with an off-axis location of the collecting optics at an angle of $30 \mathrm{deg}$. The length of the measurement volume was $1 \mathrm{~mm}$, and the diameter was $0.2 \mathrm{~mm}$. The total laser power used was $18 \mathrm{~W}$. To seed the flow, we used 1-micron-dia alumina powder particles mixed with a fused silica flowing agent Cab-0-Sil provided by Micro-Abrasive Corporation.

The alternating light intensity scattered by the passage of the particles through the measurement volume is detected by the receiving optics, filtered to separate the green and blue components, and converted into electronic signals by photomultiplier tubes. The transmitting optics incorporates an acousto-optical modulator (Bragg ce11), which causes the fringe patterns in the measurement volume to move at a constant velocity to distinguish between velocity directions. Thus, a unique frequency burst of scattered light is obtained for particles in the measurement volume having instantaneously positive, zero, or negative velocity. The high frequency signal burst from the photomultiplier tube is passed to an electronic processor that includes validation circuits. The validation circuits assure that on $1 \mathrm{y}$ signals associated with the passage of a single particle through the measurement volume are accepted as data. The data are transferred to a computer where preliminary analysis provides a graphics output of velocity histograms, mean velocity, and turbulence intensity.

The validated data are written onto a magnetic disc or magnetic tape for subsequent analysis. The correlation and spectral analysis program allows the computation of the correlation and spectral statistics of any two elements of the data and their cross-correlation and cross-spectral distribution. A unique data reduction subroutine enabling phase-locked conditional sampling and ensemble averaging, as required, for nonstationary measurements in a periodic flowfield, is also included in this laser velocimeter system. The details of this procedure are given in the next subsection.

The two laser systems mounted on two separate frames, shown in Figure 2.8 , may be traversed throughout the jet flow field in two independently computer-controlled Cartesian coordinate systems ( $x, y, z)$, a total of six different motion channels. Only one frame was used in this study.

\subsection{Conditional Sampling Procedure}

The major goal of this study was to measure the dynamics of the 1argescale structures in a high Reynolds number supersonic jet using a nonintrusive device. To accomplish this goal, a new data acquisition and reduction procedure for conditional sampling and ensemble averaging was developed for use with the Lockheed laser velocimeter. This procedure has, by now, been extensively verified by applying it to propeller flow fields (Ref. 2.6) and low speed, acoustically excited jets (Ref. 2.7). A brief description of the procedure is given below. 
The laser velocimeter measures flow velocity by measuring the transit time of a particle moving with the flow through the measurement volume. The random distribution of the particles in the fluid causes the velocity measurements to be taken at nonuniform time intervals. Any processing of the laser-velocimeter signal using conditional sampling must account for this random nature, as it prevents the sampling of the velocity signal at prescribed time instants as it is customary with analog signals.

The data reduction procedure, used in this study, is schematical1y depicted in Figures 2.9 and 2.10 (Refs. 2.8, 2.9). Ensemble averaging is accomplished by synchronizing the beginning of the repetitive data acquisition intervals of the laser velocimeter with respect to the flow periodic oscillations as shown in Figure 2.9. The total time of measurement is divided into equal-time repetitive sampling intervals of length $\Delta T$, identical with the period of the flow oscillations. As seen in Figure 2.9 the limits of these intervals are marked $\mathrm{T}_{k}, \mathrm{~T}_{k+1}, \mathrm{~T}_{\mathrm{k}+2}, \ldots, \mathrm{T}_{k+m}$. In the case of a tone excited jet, the frequency of these limits or triggering marks is equal to the excitation frequency. The marks are recorded among the laser velocimeter data as the velocity signal of a prescribed value.

During the postprocessing procedure, each of the repetitive sampling intervals is further subdivided into a specified number of time slots, with limits marked $\tau_{0}, \tau_{1}, \tau_{2}, \ldots, \tau_{j}$ in each of the repetitive sampling intervals. While' the time period of the triggering marks is controlled by the periodicity of the flow phenomena under investigation, the number of time slots in the repetitive sampling intervals is optional, is kept the same for all the sampling intervals, and can be reassigned a different value during postprocessing of the data.

The laser velocimeter data allocated to time slots within the sampling intervals can now be used to reconstruct the velocity and turbulence intensity time histories. This is accomplished by taking data over all sampling intervals and assigning all the acquired data points in corresponding time slots within the single resulting file, as indicated in Figure 2.10. A mean value of a velocity within each time slot is then computed, and this value is allocated to the center of each of the time slots, as is also shown in Figure 2.10. This ensemble average gives the desired velocity time history over a time interval $\Delta \mathrm{T}$. The velocity time history represents, in fact, the changes in the flow velocity due to the organized large-scale structure, because the random, turbulent fluctuations are strongly suppressed by the averaging process. The velocity time history is, in general, a periodic curve, and can be further processed to obtain the probability distribution, variance and root mean square value, and higher order moments. The data are also amenable to digital Fourier analysis to obtain their frequency content.

Furthermore, it is possible to compute a root mean square value of each of the independent data sets allocated to the individual time slots. The mean value of each of the sets is the ensemble averaged velocity mentioned above. Similarly, a root mean square value of the set with respect to its mean value is a measure of the fluctuation intensity associated with the random portion of the velocity signal at the time instant corresponding to the given phase of 


\section{DATA ACQUISITION PROCEDURE}
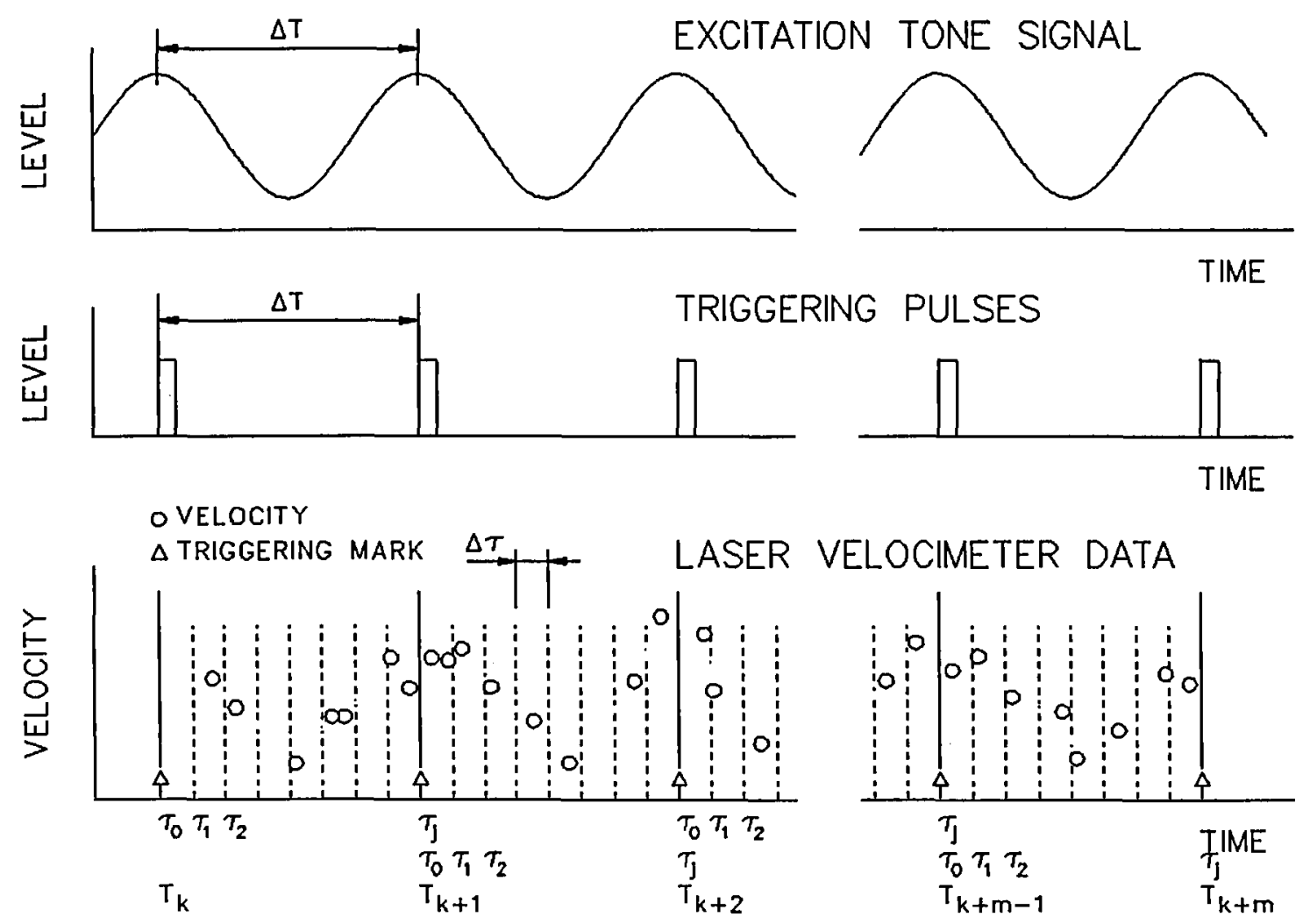

Figure 2.9 Data acquisition procedure. 


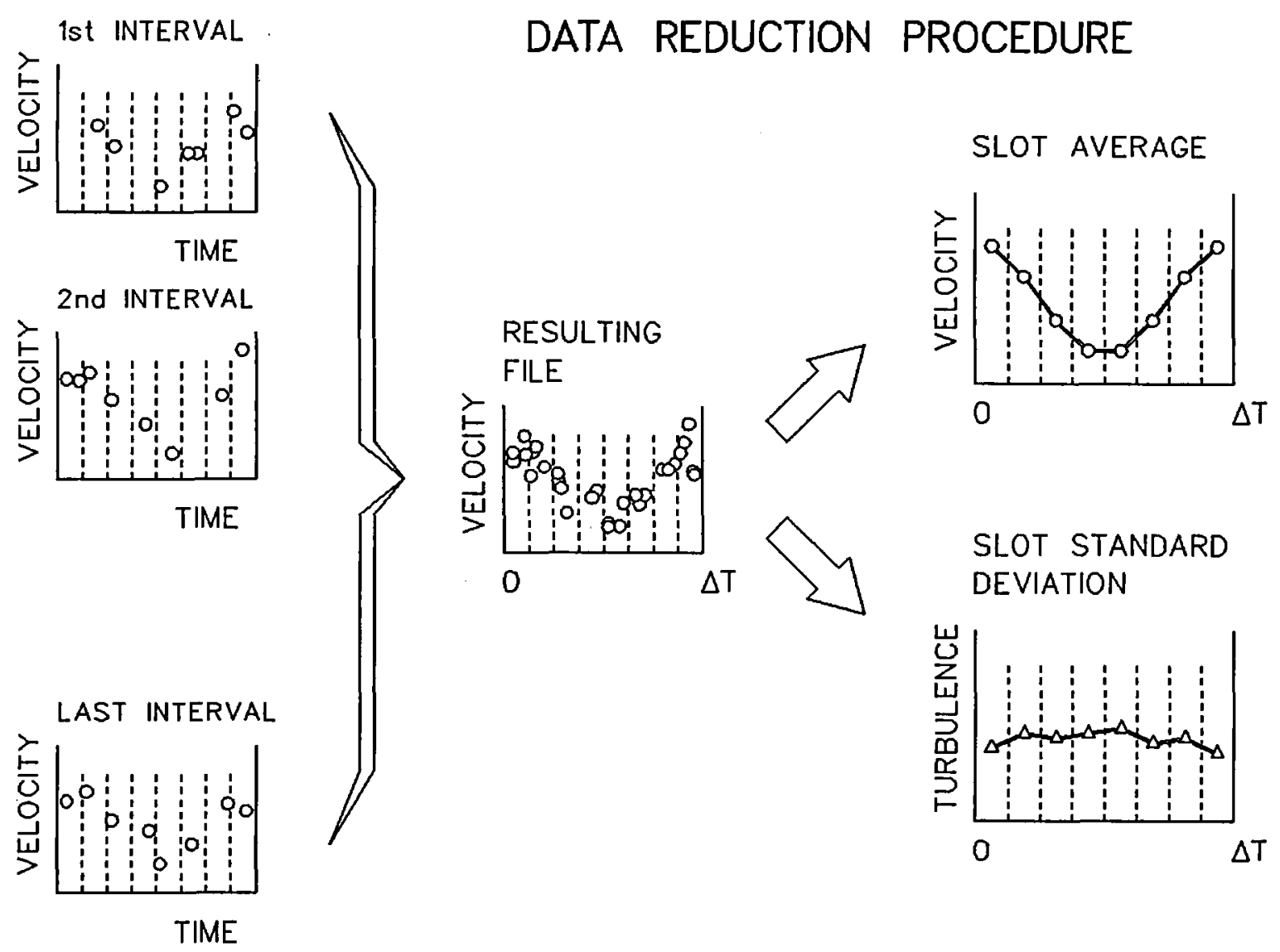


the periodic portion of the velocity signal. Thus, plotting the root mean square values for each of the time slots of the resulting time interval gives the fluctuation intensity distribution of the random portion of the velocity signal (turbulence intensity history) over the sampling interval.

\subsection{Test Conditions}

The research program consisted of the following two tasks:

Task I. Flow Visualization of Large-Scale Structure

Task II. LV Quantitative Measurements of Large-Scale Structures

A11 tests were conducted using a 50.8-mm-dia C-D nozzle operated at its design Mach number of 1.37. A11 flow visualization data were obtained without heating the jet, whereas for the LV data, the jet had to be heated mildly (reservoir temperature $=370 \mathrm{~K}$ ) to render it into an isothermal jet. This was necessitated by the fact that the supersonic jet when operated unheated contains a large number of condensate particles, which produce a considerable amount of background scattering of the laser light during the LV measurements, thus producing very low $S / N$ ratio.

Other test details for each task are described below.

\subsubsection{Task I - F1ow Visua1ization of Large-Scale Structures}

For these tests, real time measurements were first made at a range of acoustic frequencies. At each frequency, the input voltage to the acoustic drivers was increased gradually, until the large-scale structure became just visible in the jet.

Photographically averaged schlieren records of the jet flow structure were acquired at excitation Strouhal numbers $S_{e}=f D / U=0.2,0.3,0.4,0.5$, $0.6,0.7$, and 0.8 .

To determine the phase velocity of the large-scale structure, each photograph was taken with a 60 deg (or smaller in some cases) phase delay between the trigger signal and the light strobing.

\subsubsection{Task II - LV Quantitative Measurements of Large-Scale Structures}

\section{Base1ine Experiments}

Conventional laser velocimetry measurements without acoustic excitation were obtained first to define the nature of the evolving jet shear layer. Several of these measurements are essential to current quasi-linear instability theories, and these measurements consisted of the following:

1. Mean axial velocity component.

2. Root-mean-square value of axial turbulence velocity component. 
Both items were acquired at various axial locations from the nozzle exit plane to 20 jet diameter. These measurements were made along the jet centerline. Radial profiles of both items were also measured at four axial locations.

\section{Frequency Response}

To determine the frequency response of the laser velocimetry system, five axial component turbulent spectra were obtained, evenly divided along the jet lipline and covering the initial mixing layer. These spectra were collapsed to a universal shape in terms of the local turbulence Strouhal number. This collapse was considered as sufficient evidence for determining adequate frequency response for these laser velocimetry measurements. (For reasons given in Section 5.0 , these data were acquired for $M_{j}=0.9$. The results are
also presented in Section 5.0.)

\section{Conditiona11y Sampled LV Measurements}

The laser velocimeter measurements were based on the conditionally sampled method described in subsection 2.6. Only one excitation Strouhal number was required for this program. The selected Strouhal number for excitation was determined as the most preferential mode from the Task I flow visualization study.

We started out by trying to acquire the conditionally sampled laser velocimetry data to determine the following:

1. Root-mean-square value of phase averaged component.

2. Root-mean-square value of turbulence component.

3. Azimuthal behavior of phase averaged component.

4. Phase velocity of phase averaged component.

However, as explained in Section 4.0 and 5.0, the measurements were not as exhaustive as planned, and only partial information for each of the above four items could be determined in the long run. 


\subsection{FLOW VISUALTZATION RESULTS}

This section on results of the flow visualization task is divided into four subsections. The first two subsections describe the results of the excitation-level and frequency-effects investigation, respectively. The results of time delay experiments to determine the spatial development of the large-scale structure are presented in the third subsection. The last subsection summarizes the conclusions. It should be pointed out that detailed video records of the flow visualization were also made, and two copies of the video cassette are being provided to NASA Langley along with this report.

\subsection{Excitation Leve1 Effects}

The excitation-level effect experiments were performed at the outset of the flow visualization task to determine the excitation levels needed to just lift up the jet large-scale structure from within the background, dominantly random turbulence, so as to be distinctly visible. The root mean square voltages fed to the acoustic drivers were used to denote the strength of excitation, instead of true excitation sound pressure levels; because it was found difficult to make reliable measurements of the excitation levels at the nozzle exit plane of the supersonic jet.

In some cases, however, the sound pressure levels, $L_{e}$, were measured just outside the nozzle lip with the jet running and, where available, these levels have been shown in the figures.

The excitation-level results, were obtained at the excitation frequency of $\mathrm{f}_{\mathrm{e}}=3128 \mathrm{~Hz}$, which corresponds to the jet excitation strouhal number of $\mathrm{s}_{\mathrm{e}}$ $=0.4$. As shown in the next subsection, this excitation strouhal number was found to be the most efficient one to excite large-scale structures in the shear layer of the supersonic jet of $\mathrm{M}_{j}=1.37$. It should be mentioned here, that the frequency of the strobing for phase-locked visualization was identical to the excitation frequency. The same triggering frequency for photographic ensemble averaging was used also in the case of the unexcited supersonic jet.

The unexcited jet is shown in Figure 3.1. As seen in this figure, the jet shear layer width increases smoothly as flow progresses in the downstream direction. No distinctive periodic large-scale structure, synchronous with the triggering frequency $(3128 \mathrm{~Hz})$, is traceable in the shear layers in this figure.

The ensemble-averaged photographs of the jet excited at different excitation voltages are shown in Figures 3.2 through 3.4. As seen in these pictures, on raising the excitation voltage from $15 \mathrm{~V}$ to $19 \mathrm{~V}$, the periodic large-scale structure in the shear layer of the supersonic jet becomes gradually more distinguishable. At the highest excitation voltage of $19 \mathrm{~V}$ (Figure 3.4), the large-scale structure is distinctly visible.

Based on the above-described experiment, it was decided to maintain the excitation voltage at a level of $19 \mathrm{~V}$ throughout the experimental program for 


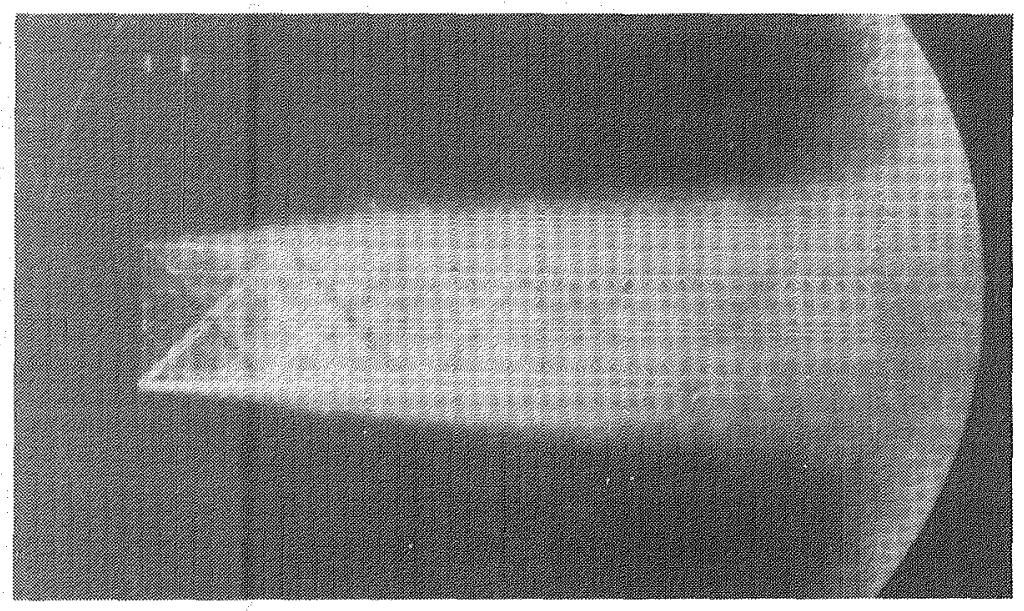

Figure 3.1 Ensemble-averaged photograph of an unexcited jet. (Knife edge: Vertical, $\mathrm{f}_{\mathrm{s}}=3125 \mathrm{~Hz}, \phi=0^{\circ}$ )

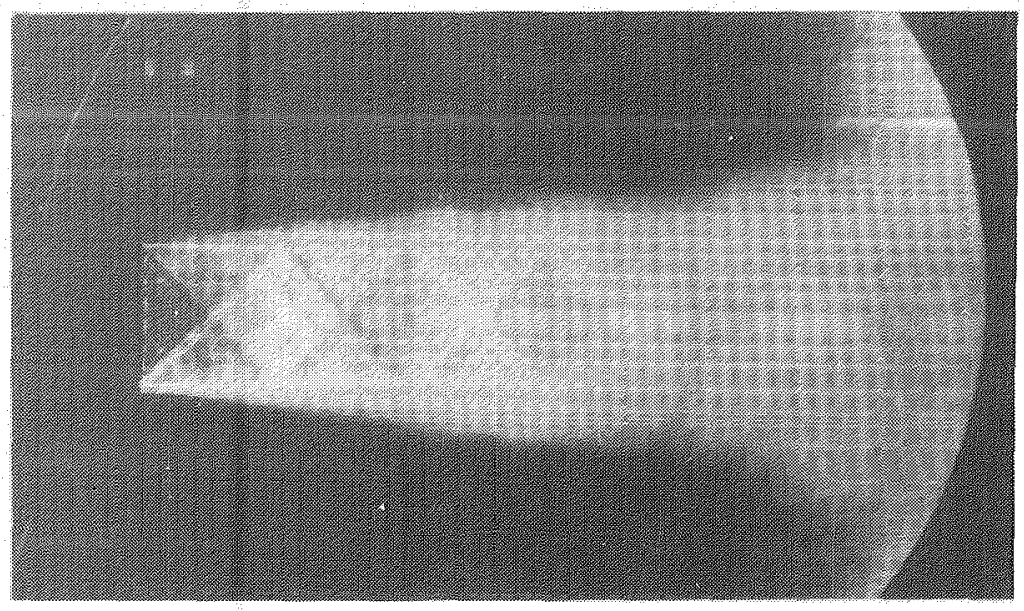

Figure 3.2 Ensemble-averaged photograph of an excited jet. (Knife edge: Vertical, $\mathrm{M}_{\mathrm{j}}=1.37, \mathrm{~S}_{\mathrm{e}}=0.4, \mathrm{~V}_{\mathrm{D}}=15 \mathrm{~V}$, $\left.f_{S}=3116 \mathrm{~Hz}, \phi=0^{\circ}\right)$ 


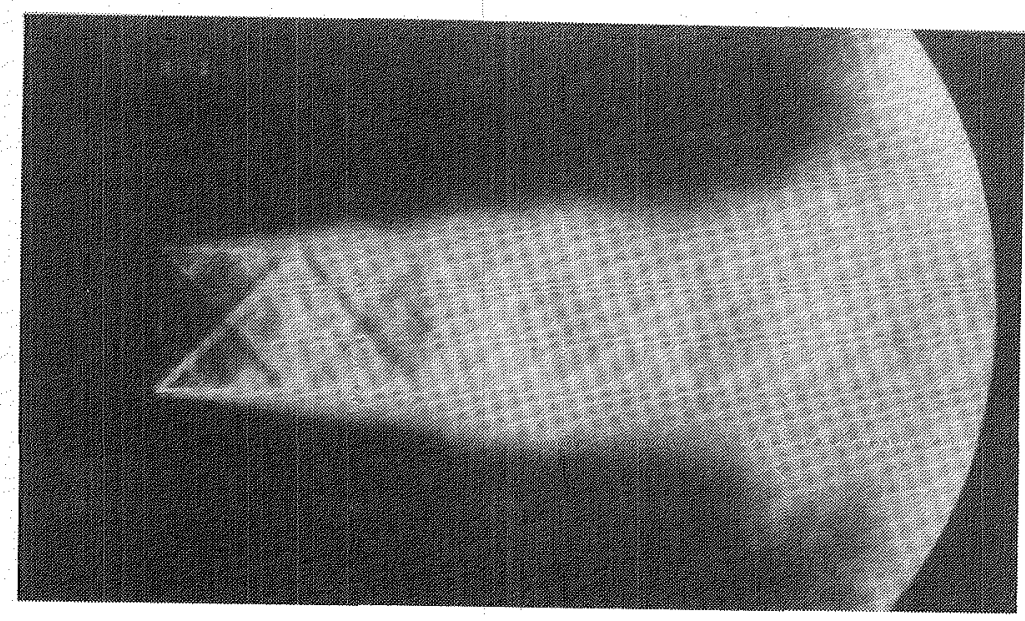

Figure 3.3 Ensemble-averaged photograph of an excited jet. (Knife edge: Vertical, $\mathrm{M}_{\mathrm{j}}=1.37, \mathrm{~S}_{\mathrm{e}}=0.4, \mathrm{~V}_{\mathrm{D}}=17.5 \mathrm{~V}$,
$\mathrm{f}_{\mathrm{S}}=3112 \mathrm{~Hz}, \phi=0^{\circ}$ )

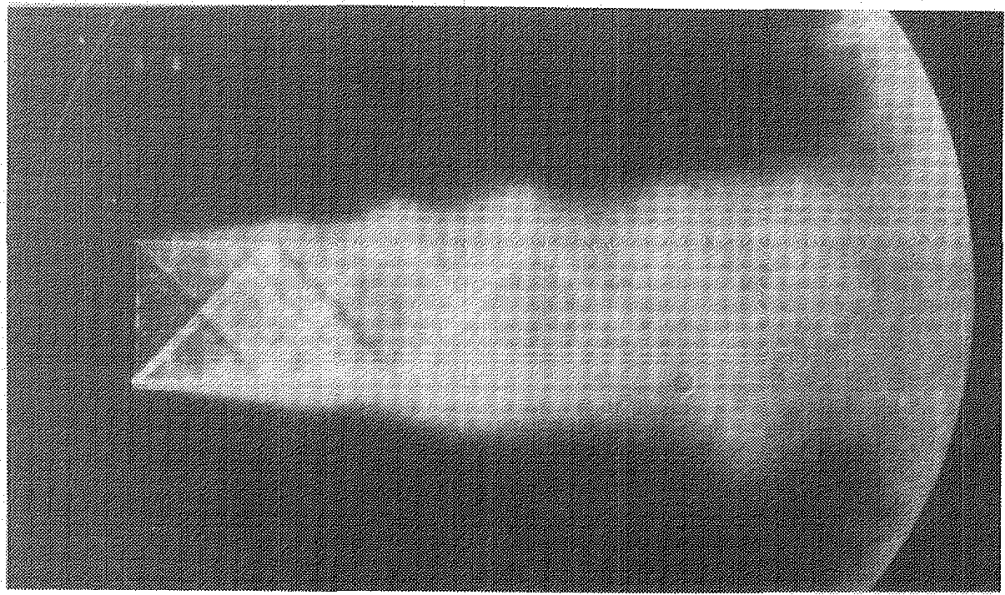

Figure 3.4 Ensemble-averaged photograph of an excited jet.

(Knife edge: Vertical, $\mathrm{M}_{\mathrm{j}}=1.37, \mathrm{~S}_{\mathrm{e}}=0.4, \mathrm{~V}_{\mathrm{D}}=19 \mathrm{~V}$, $f_{\mathrm{S}}=3122 \mathrm{~Hz}, \phi=0^{\circ}$ )

$\mathrm{L}_{\mathrm{e}}=119 \cdot \mathrm{dB}$ (at the nozzle lip1ine) 
all excitation frequencies. This excitation voltage also turned out to be the maximum voltage permissible for a continuous and reliable operation of our source section. It should also be pointed out that the actual excitation levels at constant excitation voltage of $19 \mathrm{~V}$ at different excitation frequencies may vary due to non-flat frequency response of the driver system and also non-flat sound transmission response of the duct system.

\subsection{Excitation Frequency Effects}

The excitation Strouhal number (excitation frequency) experiments were aimed at determining the most preferential strouhal number for the coherent large-scale structures in the shear layer of a supersonic jet of $M_{j}=1.37$. The jet was excited in a frequency range of $f_{e}=1547-6228 \mathrm{~Hz}$. This frequency range corresponds to the excitation strouhal number range of $\mathrm{s}_{\mathrm{e}}=$ $0.2-0.8$. The excitation voltage was maintained at $19 \mathrm{~V}$ at a11 excitation frequencies, as stated in the previous subsection.

Ensemble averaged schlieren pictures of the fully-expanded, supersonic jet of $\mathrm{M}_{j}=1.37$ for different triggering frequencies are shown in Figures 3.5 through 3.11. These figures cover the flow structure development along the first 6 nozzle exit diameters. It should be noted here once more, that the triggering frequencies for photographic ensemble averaging and the flow excitation frequencies are equal for each particular excitation frequency. The Strouhal number of 0.2 does not appear to support a formation of the large-scale structure in the jet, as seen in Figure 3.5. A smooth development of the jet shear layer resembles the unexcited jet shown in Figure 3.1, however, it seems that the shear layer of the excited jet (Figure 3.5) spreads somewhat faster and jet plume is wider than the unexcited jet.

On raising the excitation strouhal number from 0.2 to 0.4 , the appearance of the periodic large-scale structure becomes obvious. At the Strouhal number of 0.3 (Figure 3.6), two "humps" along the jet shear layer boundary are visible. This indicates the presence of a periodic structure in the flow synchronous with the triggering frequency. At the Strouhal number of 0.4 (Figure 3.7), an increased number of humps is present in the jet shear layer. The wave length of the excited large-scale structure in Figure 3.7 is smaller than that in Figure 3.6, which corresponds to the higher excitation frequency in the case of Figure 3.7. At excitation Strouhal numbers ranging from $\mathrm{S}_{\mathrm{e}}=$ 0.5 to $\mathrm{S}_{\mathrm{e}}=0.8$ (Figures 3.8 through 3.11 ), the formation of the large-scale structure is not as clear as in the two previous cases. Also, especially at two highest Strouhal numbers, jet plume seems to be narrower than it was at low excitation Strouhal numbers. This may be a consequence of the changed level of excitation. (Due to the non-flat frequency response of the acoustic drivers, the excitation levels are lower at the high frequency end.)

The data described above covered a jet axial extent of about six jet diameters. The results of flow visualization covering the region between 6 to 12 nozzle exit diameters are shown in Figures 3.12 through 3.15 . It should be noted that only one half of the shear layer is visualized here. It was found that if the sensitivity of the optical system was adjusted to visualize the shear layer in both the upper and the lower halves of the jet, one half was 


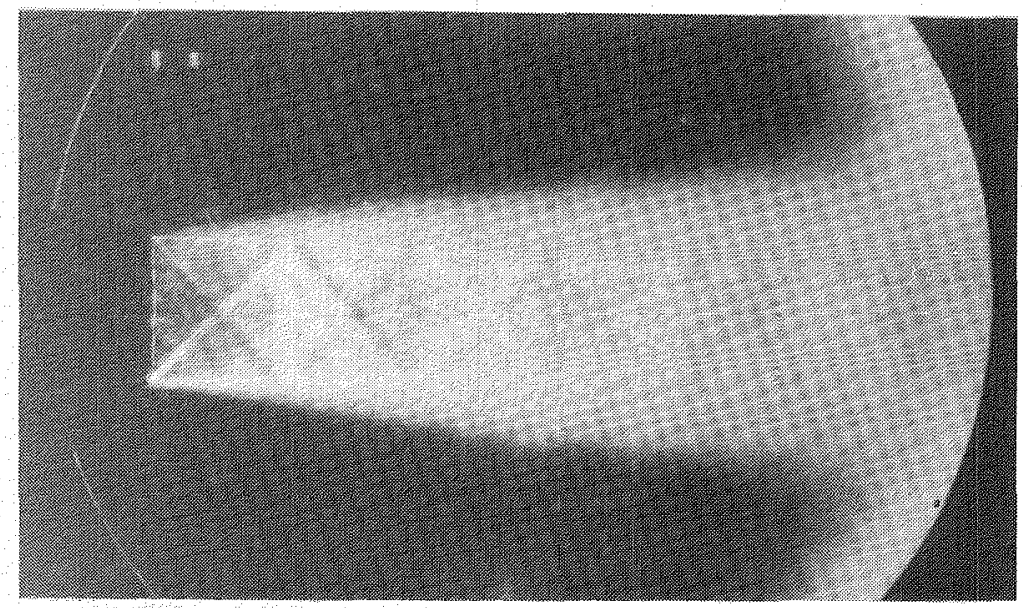

Figure 3.5 Ensemble-averaged photograph of an excited jet. (Knife edge: Vertical, $M_{j}=1.37, S_{e}=0.2, V_{D}=19 \mathrm{~V}$,
$\mathrm{f}_{\mathrm{S}}=1547 \mathrm{~Hz}, \phi=0^{\circ}$ )

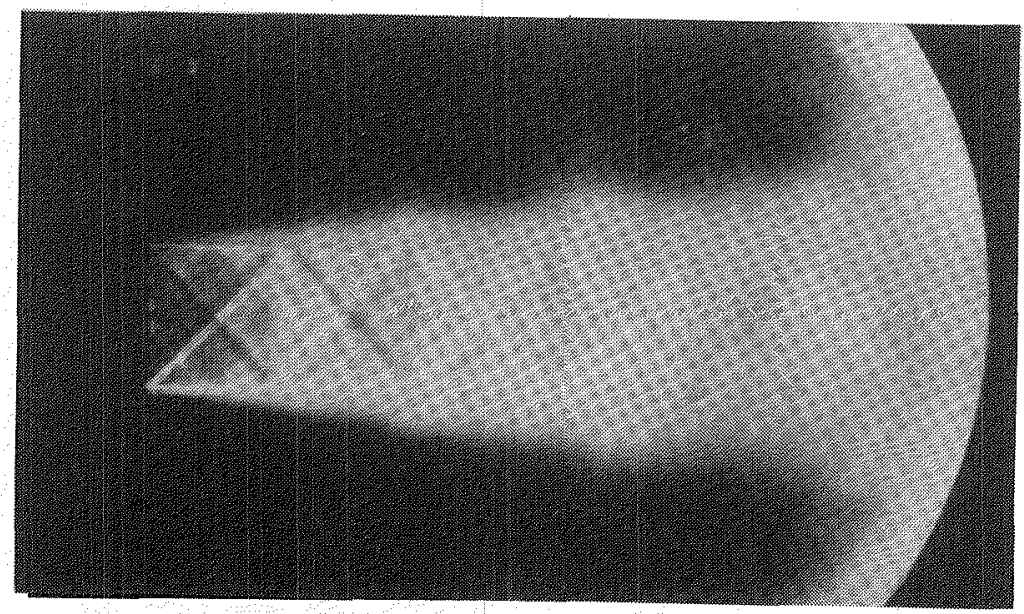

Figure 3.6 Ensemble-averaged photograph of an excited jet. (Knife edge: Vertical, $\mathrm{M}_{\mathrm{j}}=1.37, \mathrm{~S}_{\mathrm{e}}=0.3, \mathrm{~V}_{\mathrm{D}}=19 \mathrm{~V}$,
$\mathrm{f}_{\mathrm{s}}=2348 \mathrm{~Hz}, \phi=0^{\circ}$ ) 


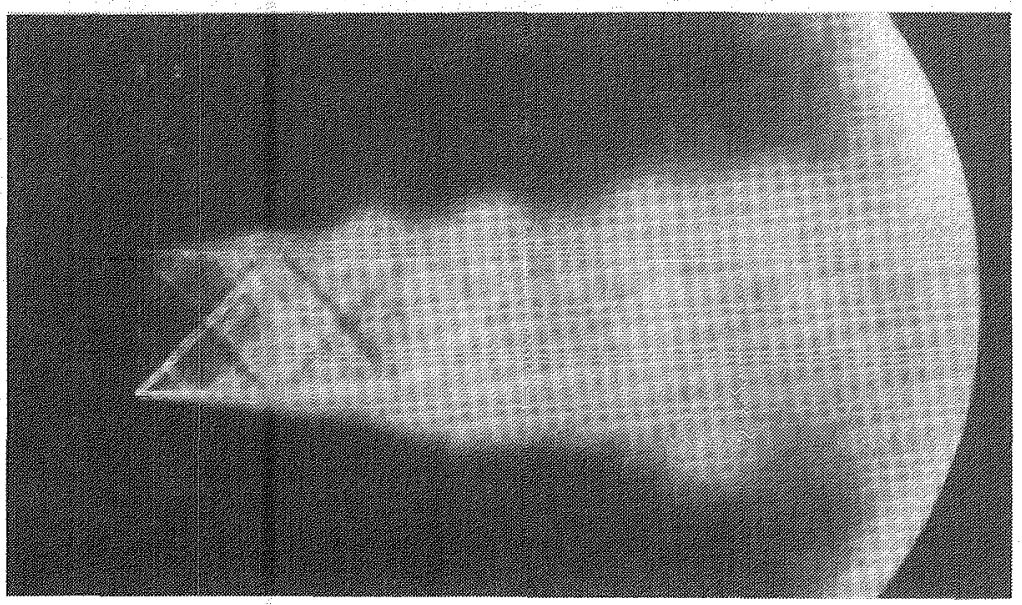

Figure 3.7 Ensemble-averaged photograph of an excited jet.

(Knife edge: Vertical, $\mathrm{M}_{j}=1.37, \mathrm{~S}_{\mathrm{e}}=0.4, \mathrm{~V}_{\mathrm{D}}=19 \mathrm{~V}$, $\mathrm{f}_{\mathrm{S}}=3128 \mathrm{~Hz}, \phi=0^{\circ}$ )

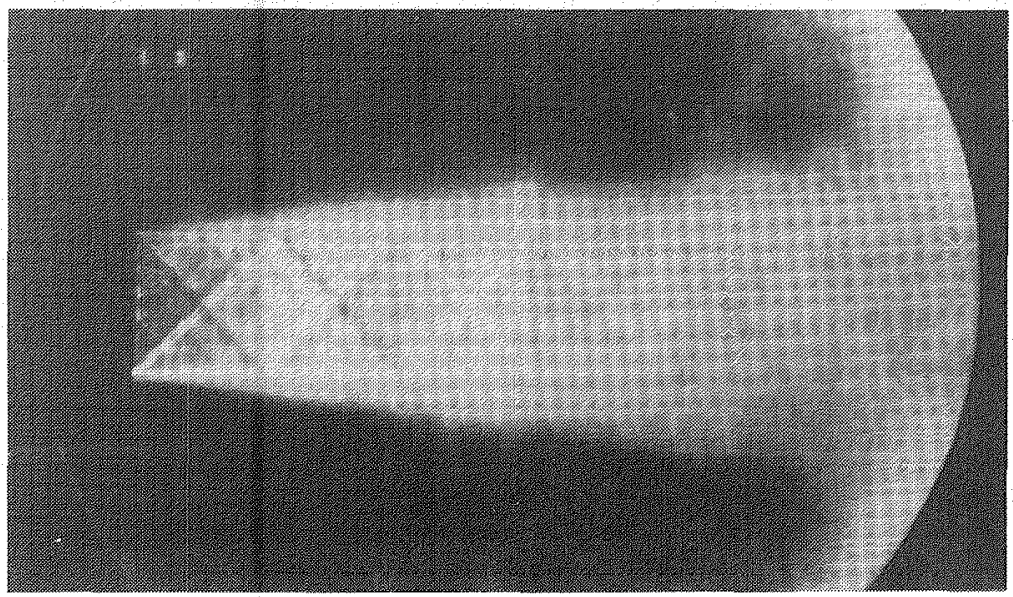

Figure 3.8 Ensemble-averaged photograph of an excited jet. (Knife edge: Vertical, $M_{j}=1.37, S_{e}=0.5, V_{D}=19 \mathrm{~V}$,
$f_{S}=3886 \mathrm{~Hz}, \phi=0^{\circ}$ ) 


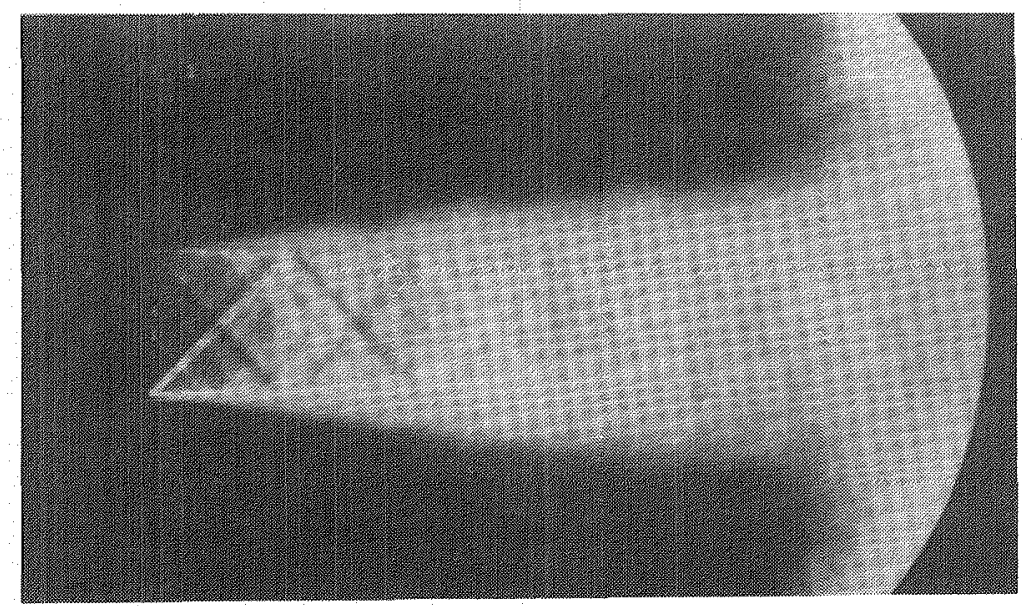

Figure 3.9 Ensemble-averaged photograph of an excited jet.

(Knife edge: Vertical, $\mathrm{M}_{j}=1.37, \mathrm{~S}_{\mathrm{e}}=0.6, \mathrm{~V}_{\mathrm{D}}=19 \mathrm{~V}$, $\mathrm{f}_{\mathrm{S}}=4667 \mathrm{~Hz}, \phi=0^{\circ}$

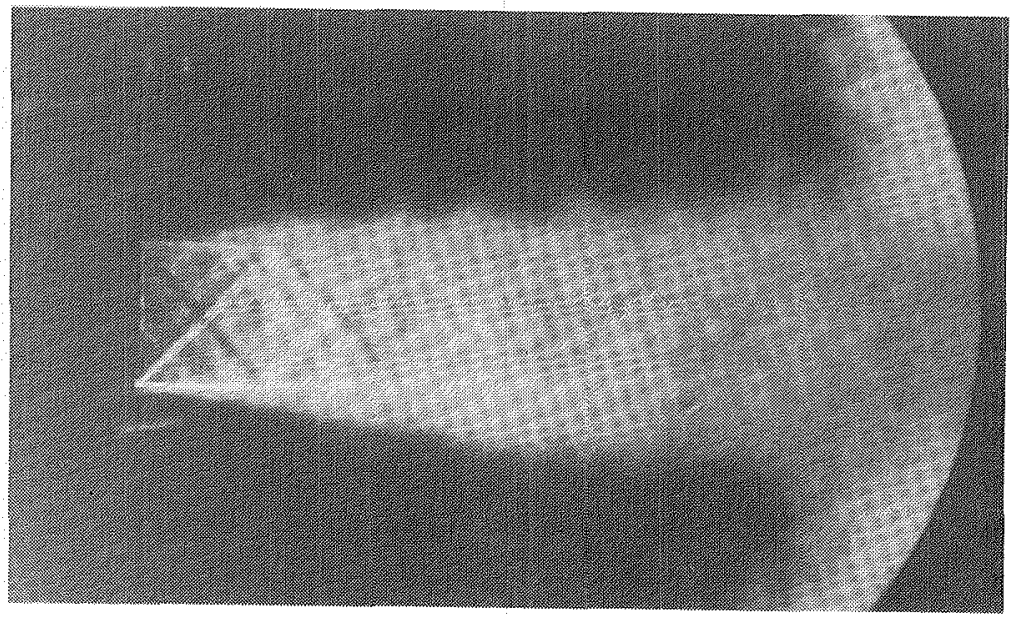

Figure 3.10 Ensemble-averaged photograph of an excited jet. (Knife edge: Vertical, $\mathrm{M}_{\mathrm{j}}=1.37, \mathrm{~S}_{\mathrm{e}}=0.7, \mathrm{~V}_{\mathrm{D}}=19 \mathrm{~V}$,
$\mathrm{f}_{\mathrm{S}}=5435 \mathrm{~Hz}, \phi=0^{\circ}$ ) 


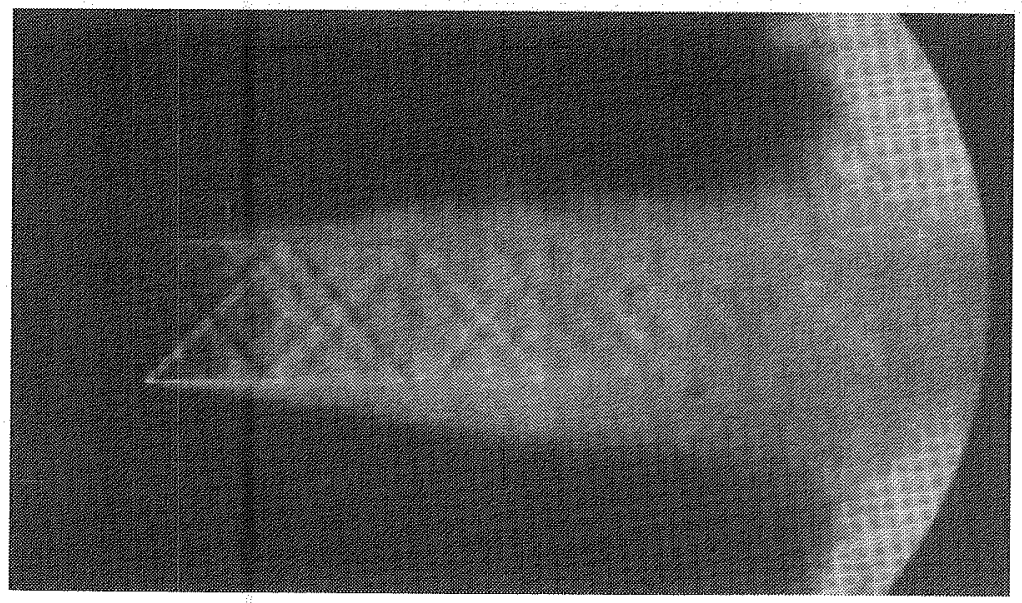

Figure 3.11 Ensemble-averaged photograph of an excited jet. (Knife edge: Vertical, $\mathrm{M}_{\mathrm{j}}=1.37, \mathrm{~S}_{\mathrm{e}}=0.8, \mathrm{~V}_{\mathrm{D}}=19 \mathrm{~V}$,
$=6228 \mathrm{~Hz}, \phi=0^{\mathrm{O}}$ ) 


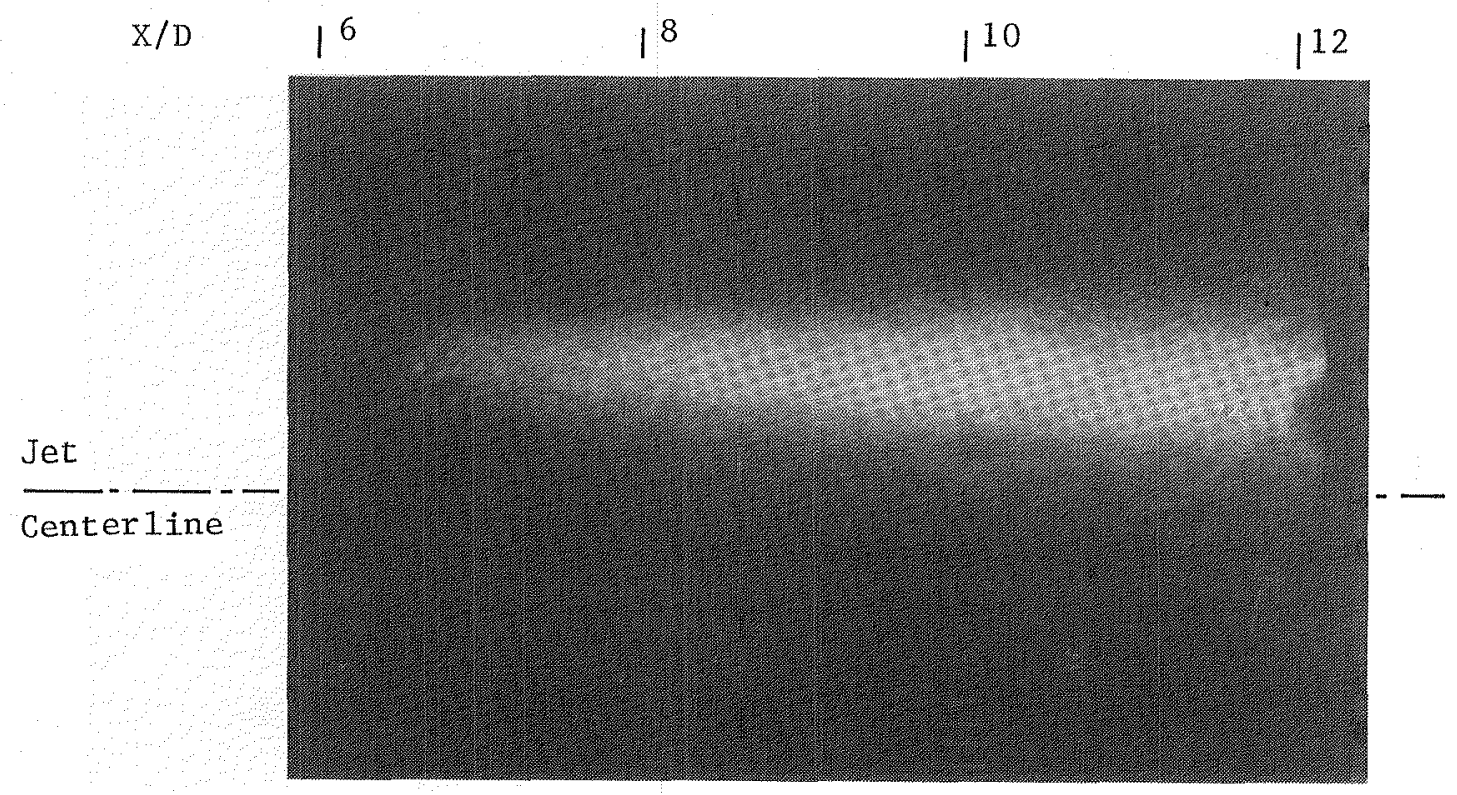

Figure 3.12 Ensemble-averaged photograph of an unexcited jet shear 1ayer.

(Knife edge: Horizontal, $M_{j}=1.37, f_{s}=3078 \mathrm{~Hz}, \phi=0^{\circ}$ )

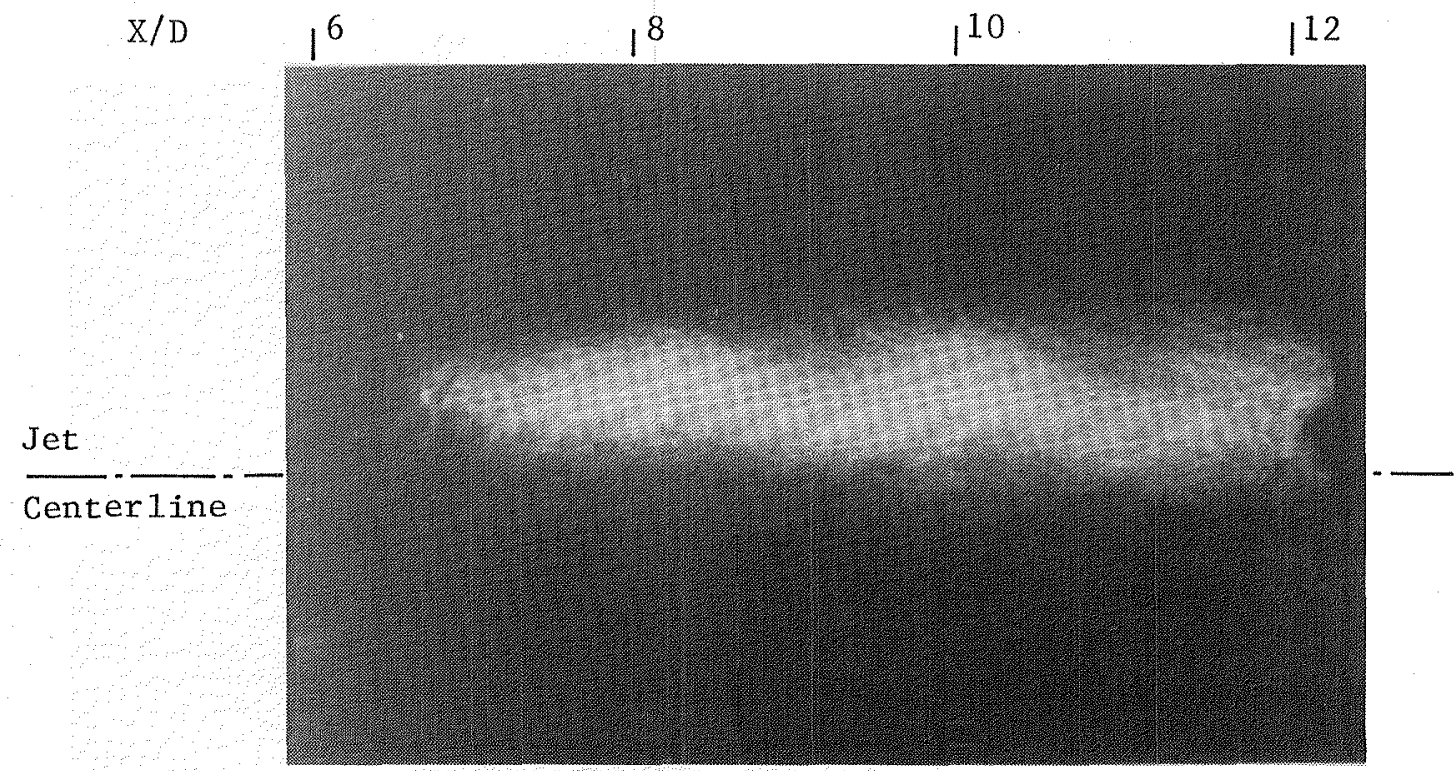

Figure3.13 Ensemble-averaged photograph of an excited jet shear layer.

(Knife edge: Horizontal, $M_{j}=1.37, \mathrm{~S}_{\mathrm{e}}=0.4, \mathrm{~V}_{\mathrm{D}}=19 \mathrm{~V}$,
$\left.\mathrm{f}_{\mathrm{s}}=3078 \mathrm{~Hz}, \phi=0^{\circ}\right)$ 


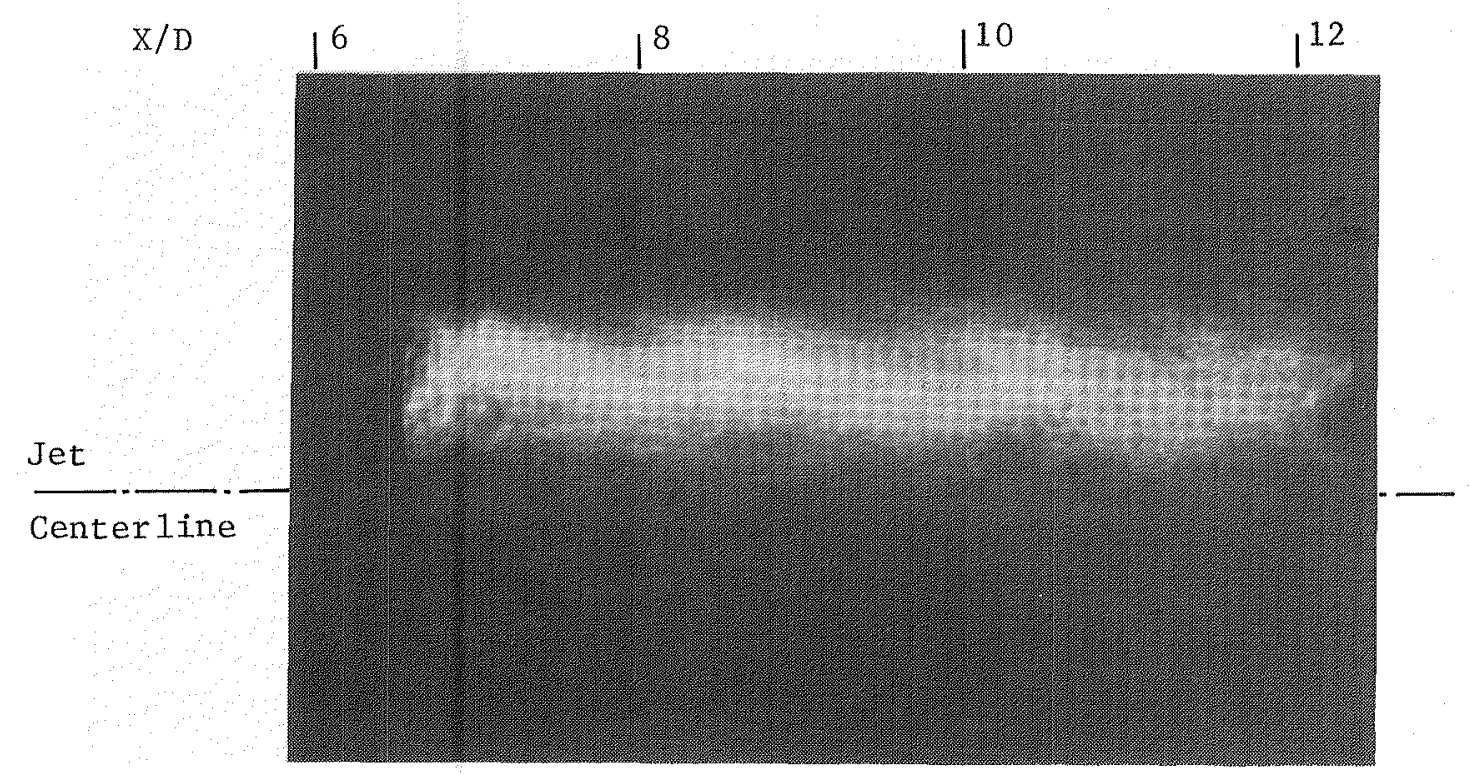

Figure 3.14 Ensemble-averaged photograph of an excited jet shear layer. (Knife edge: Horizontal, $\mathrm{M}_{\mathrm{j}}=1.37, \mathrm{~S}_{\mathrm{e}}=0.46, \mathrm{~V}_{\mathrm{D}}=19 \mathrm{~V}$,
$\mathrm{f}_{\mathrm{s}}=3566 \mathrm{~Hz}, \phi=0^{\circ}$ )

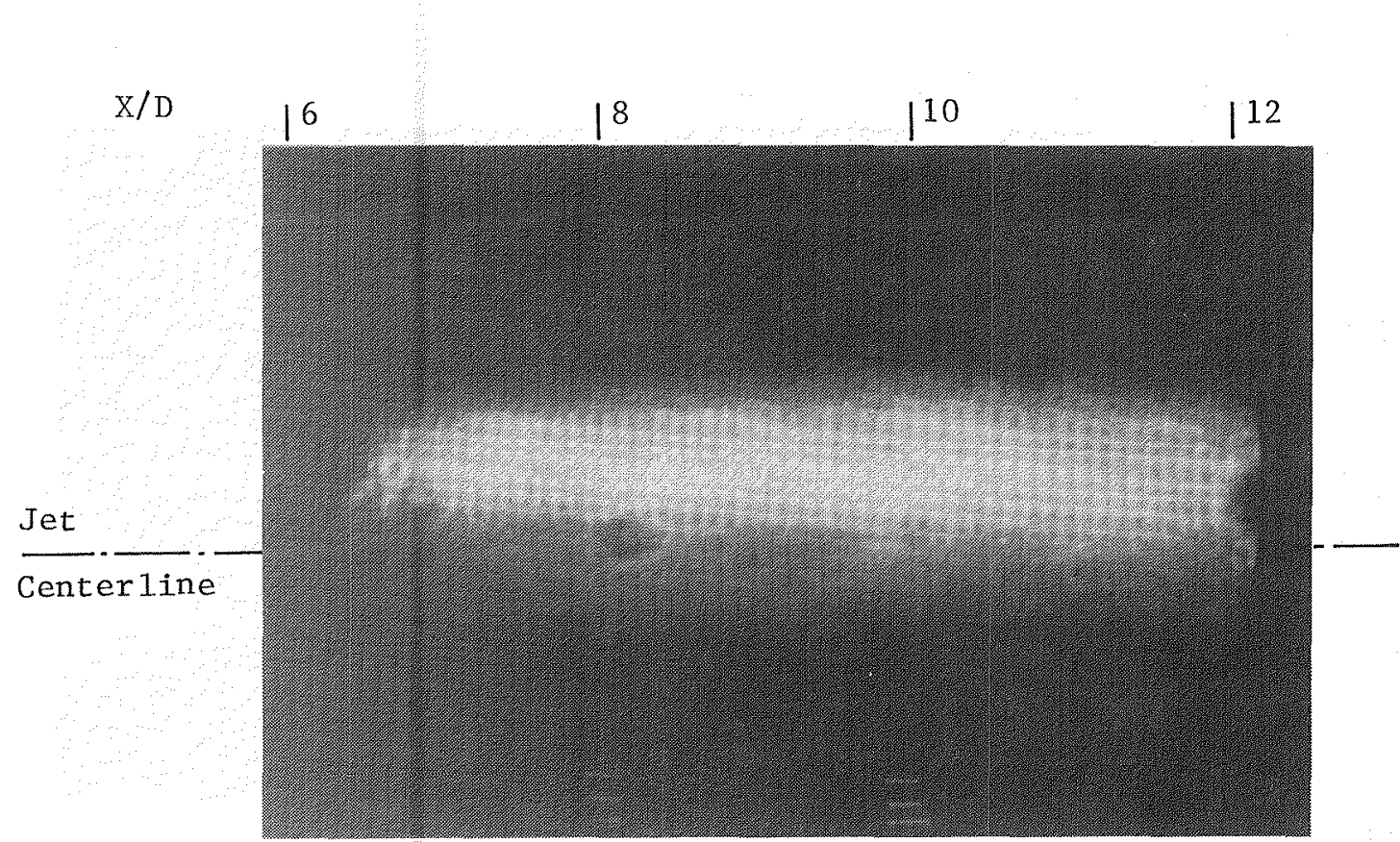

Figure 3.15 Ensemble-averaged photograph of an excited jet shear 1.ayer.

(Knife edge: Horizontal, $\mathrm{M}_{\mathrm{j}}=1.37, \mathrm{~S}_{\mathrm{e}}=0.5, \mathrm{~V}_{\mathrm{D}}=19 \mathrm{~V}$, $\mathrm{f}_{\mathrm{S}}=3950 \mathrm{~Hz}, \phi=0^{\circ}$ ) 
too bright, whereas the other was not bright enough for an adequate photograph. Thus, attempts were made to improve the quality of the visualization of one half of the jet only as shown in these figures. The following figures were taken with horizontal knife edge, and only the shear layer region of the jet is visualized.

The shear layer of the unexcited jet is shown in Figure 3.12. The ensemble-averaged photographs of the jet excited at three different Strouha1 numbers of $0.4,0.46$, and 0.5 are shown in Figures 3.13 through 3.15 . As seen in the first two pictures, the presence of the large-scale structure beyond the potential core is still traceable; the potential core extends up to $X / D=$ 7. In the case of the Strouhal number 0.5 (Figure 3.15), however, barely any changes in the flow are seen.

Based on the above-described findings, it may be concluded that the most preferential excitation Strouhal number is about 0.4 .

\subsection{Time Delay Experiments}

The time delay measurements were made to determine the development of the large-scale structure. The spatial development during one period of the triggering signal was used to calculate the large-scale structure phase velocity. The phase velocity was calculated from the distance traveled by the large-scale structure (measured on ensemble-averaged photographs) during a known time interval (phase or time delay of the light strobing with respect to the excitation signal). These measurements were made for an excitation voltage of $19 \mathrm{~V}$ and excitation Strouhal numbers $0.3,0.34,0.4,0.6$, and 0.7 .

A series of pictures with a constant phase shift of 60 degrees for a jet excited at Strouhal number of 0.3 is shown in Figure $3.16 \mathrm{a}-\mathrm{f}$. An arrow in each of the photographs indicates the instantaneous axial location of the same part of the large-scale flow structure at different phase delays. The largestructure phase velocity calculation was based on these instantaneous axial locations. In this case, this phase velocity is $276 \mathrm{~m} / \mathrm{s}$. It should be noted here, that the accuracy of phase velocity calculation from these flow visualization results strongly depends on the clarity of the images of the flow large-scale structures in the photographs. This calculation may sometimes be very crude, especially when the large-scale structure is relatively weak. Figure 3.17 a-e shows development similar to that in the previous figure, however, the phase increment in this case is 15 degrees, and the jet was excited at a strouha1 number of 0.34 . In this case, the calculated phase velocity is $260 \mathrm{~m} / \mathrm{s}$.

The phase shift interval on all remaining figures is 60 degrees. The Figures 3.18 a-e, 3.19 a-f, and 3.20 a-f show large-scale structure development in jet excited at strouha1 numbers of $0.4,0.6$, and 0.7 , respectively. The corresponding calculated phase velocities are 280,221 , and $183 \mathrm{~m} / \mathrm{s}$.

The results of time delay experiments are summarized in Figure 3.21 where the phase velocity, normalized by the nozzle exit velocity, is plotted as a 


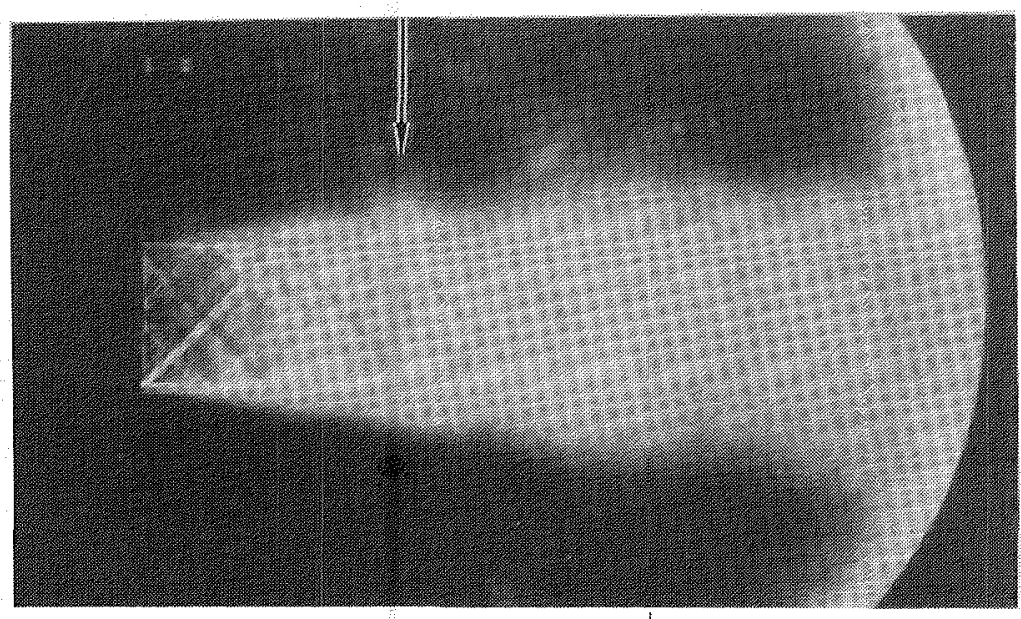

$\phi=0 \mathrm{deg}$.

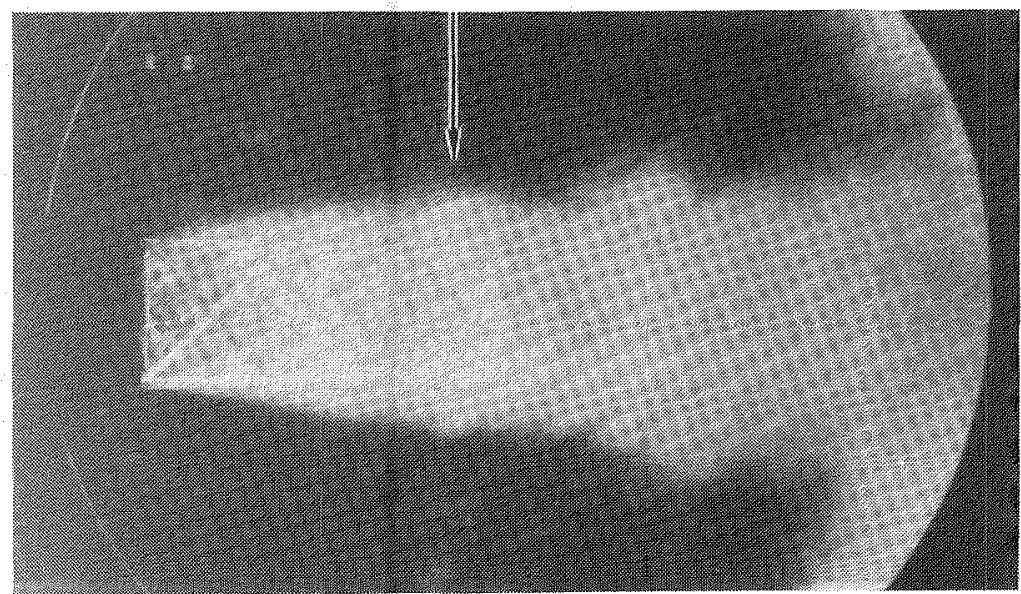

b.

$\phi=60 \mathrm{deg}$.

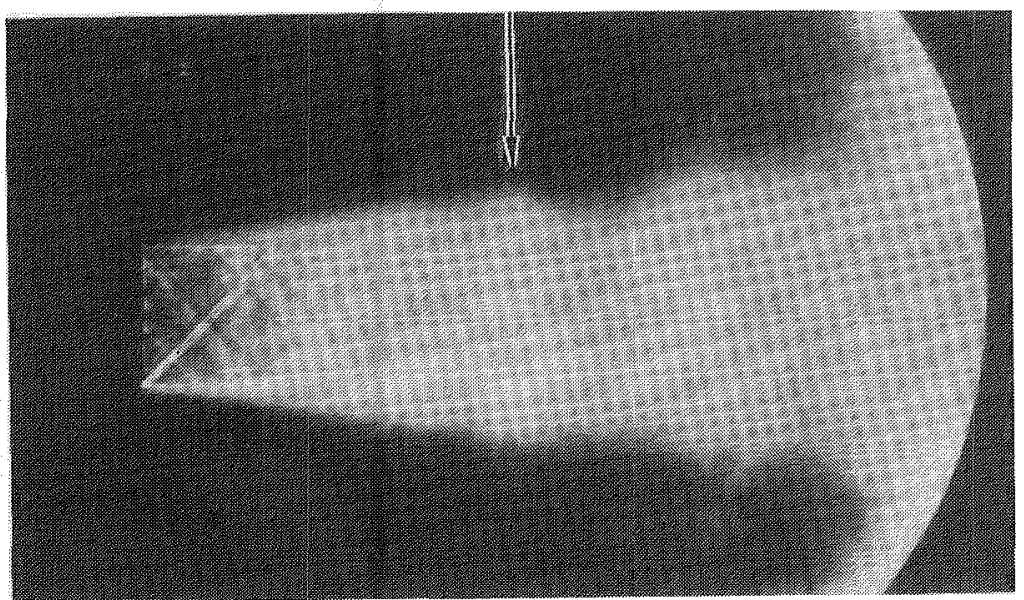

c.

$\phi=120 \mathrm{deg}$.

Figure 3.16 a-c. Ensemble-averaged photograph of an excited jet.

(Knife edge: Vertical, $\mathrm{M}_{\mathrm{j}}=1.37, \mathrm{~S}_{\mathrm{e}}=0.3, \mathrm{~V}_{\mathrm{D}}=19 \mathrm{~V}$, $\left.\mathrm{f}_{S}=2344 \mathrm{~Hz}\right)$ 


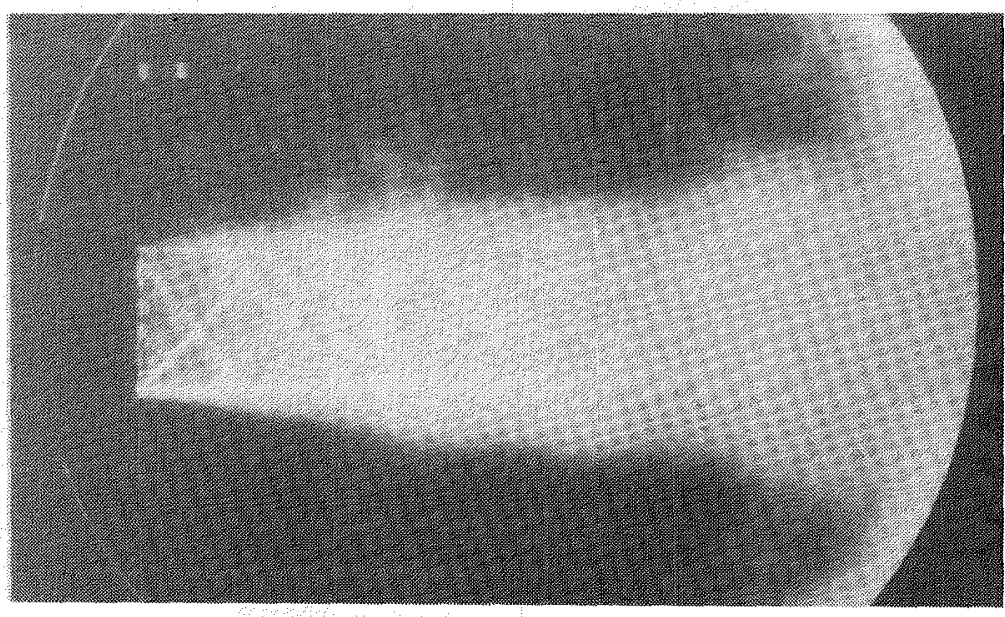

d.

$\phi=180 \mathrm{deg}$.

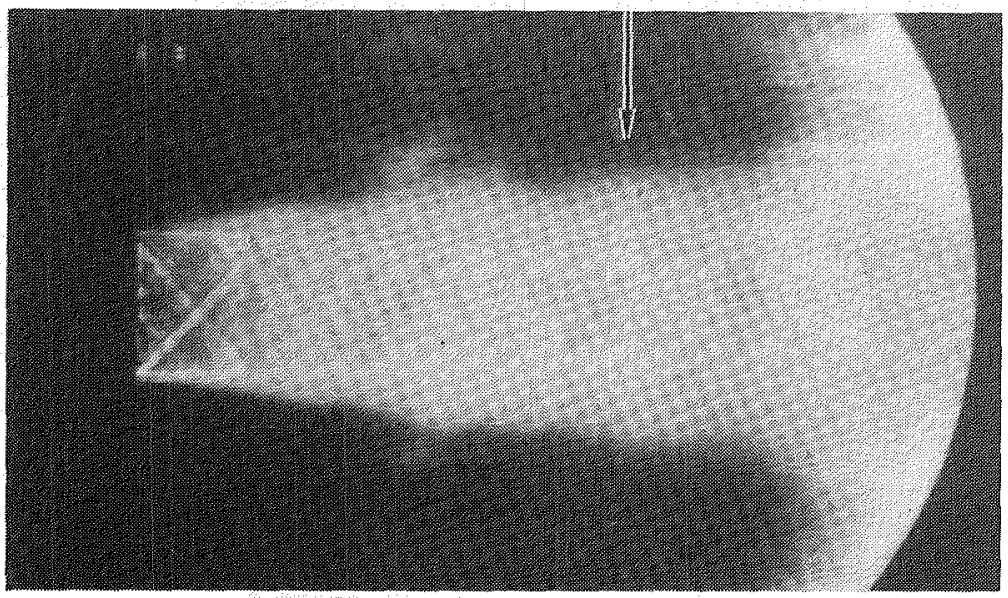

e.

$\phi=240 \mathrm{deg}$.

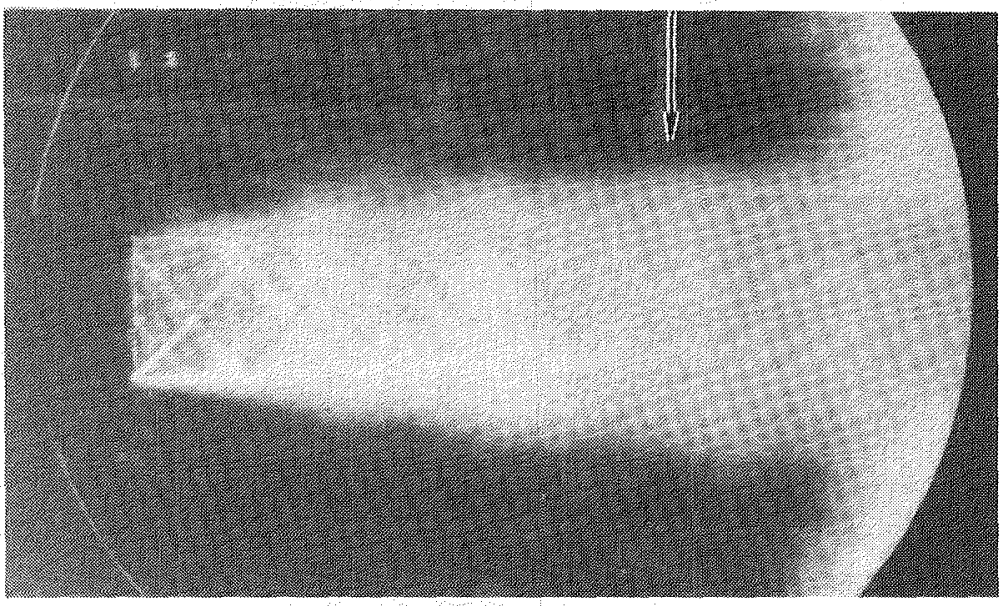

$f$.

$\phi=300 \mathrm{deg}$.

Figure $3.16 \mathrm{~d}-\mathrm{f}$. Ensemble-averaged photograph of an excited jet.

(Knife edge: Vertical, $\mathrm{M}_{\mathrm{j}}=1.37, \mathrm{~S}_{\mathrm{e}}=0.3, \mathrm{~V}_{\mathrm{D}}=19 \mathrm{~V}$, $\mathrm{f}_{\mathrm{S}}=2344 \mathrm{~Hz}$ ) 


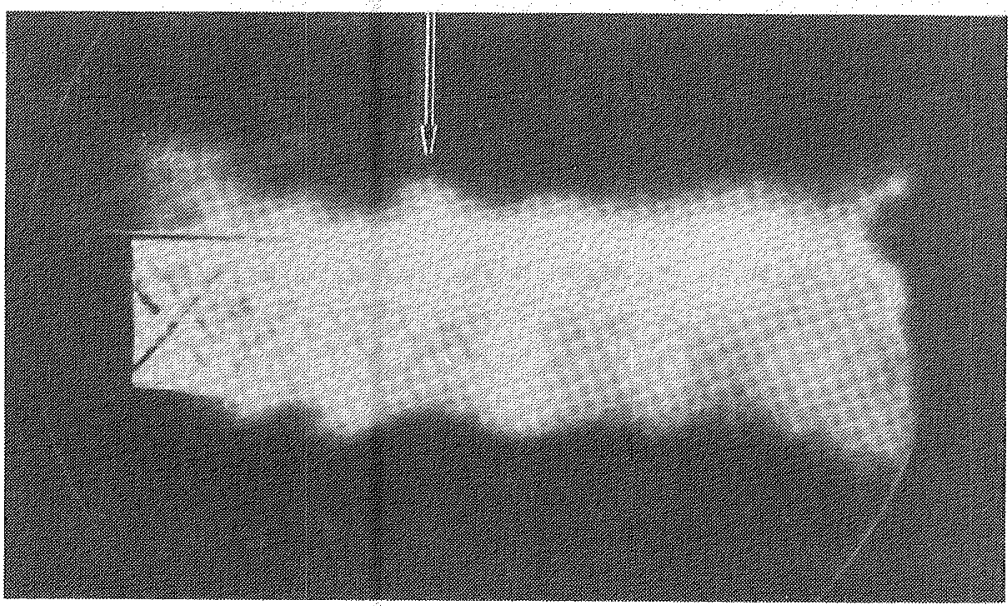

a.

$\phi=180 \mathrm{deg}$.

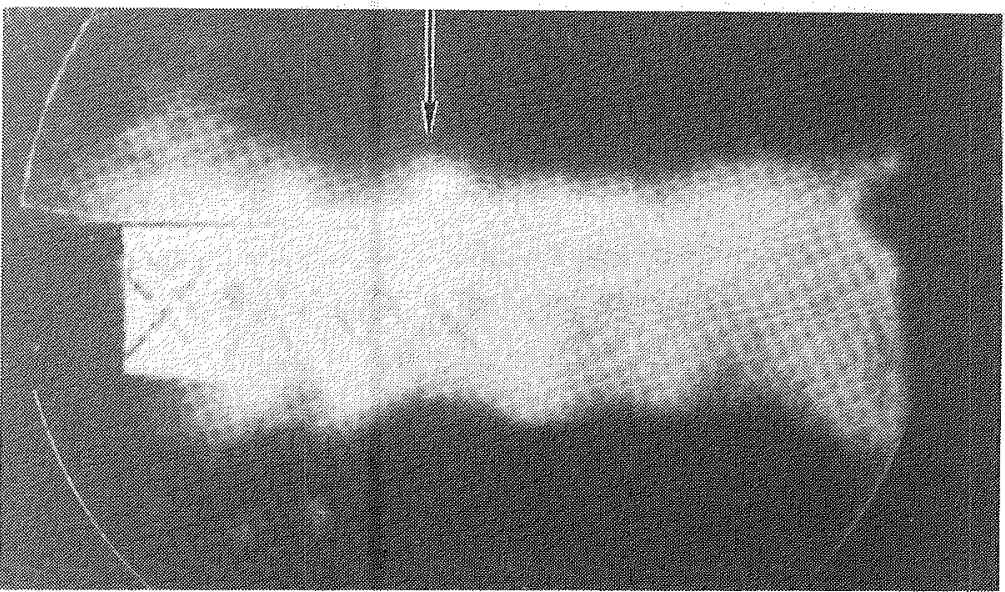

b.

$\phi=195 \mathrm{deg}$

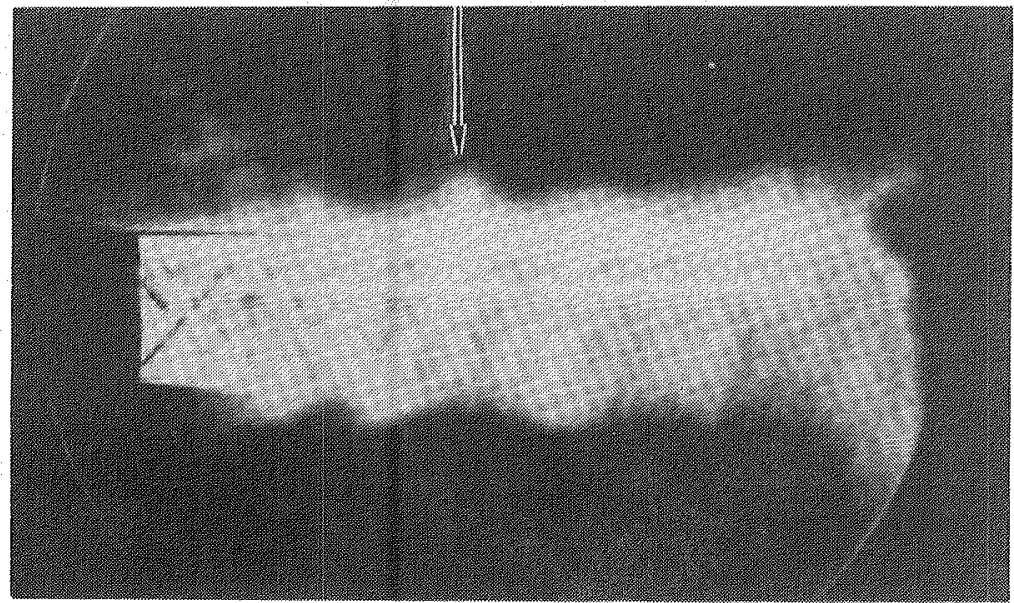

c.

$\phi=210 \mathrm{deg}$

Figure 3.17 a-c. Ensemble-averaged photograph of an excited jet.

(Knife edge: Vertical, $\mathrm{M}_{\mathrm{j}}=1.37, \mathrm{~S}_{\mathrm{e}}=0.34, \mathrm{~V}_{\mathrm{D}}=19 \mathrm{~V}$, $\left.f_{S}=2636 \mathrm{~Hz}\right)$ 


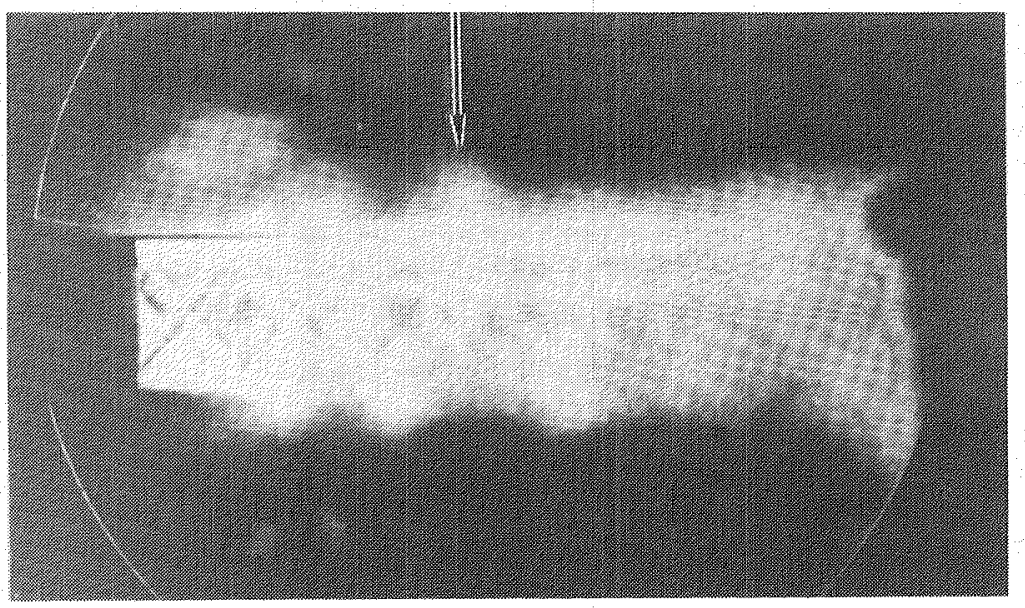

d.

$\phi=225 \mathrm{deg}$.

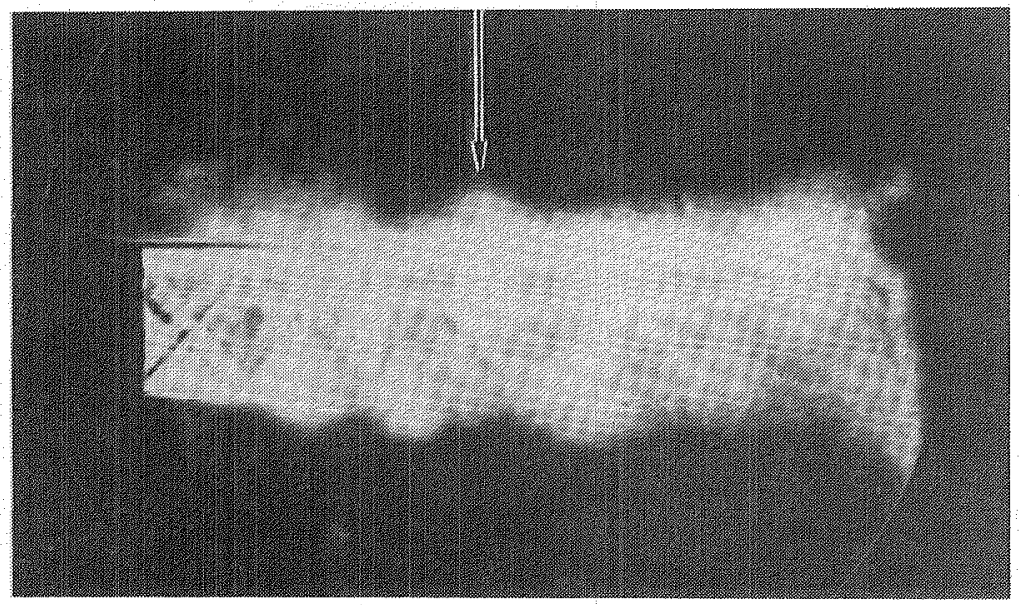

e.

$\phi=240 \mathrm{deg}$.

Figure 3.17 d-e. Ensemble-averaged photograph of an excited jet.

(Knife edge: Vertical, $M_{j}=1.37, S_{e}=0.34, V_{D}=19 \mathrm{~V}$, $\mathrm{f}_{\mathrm{S}}=2636 \mathrm{~Hz}$ ) 


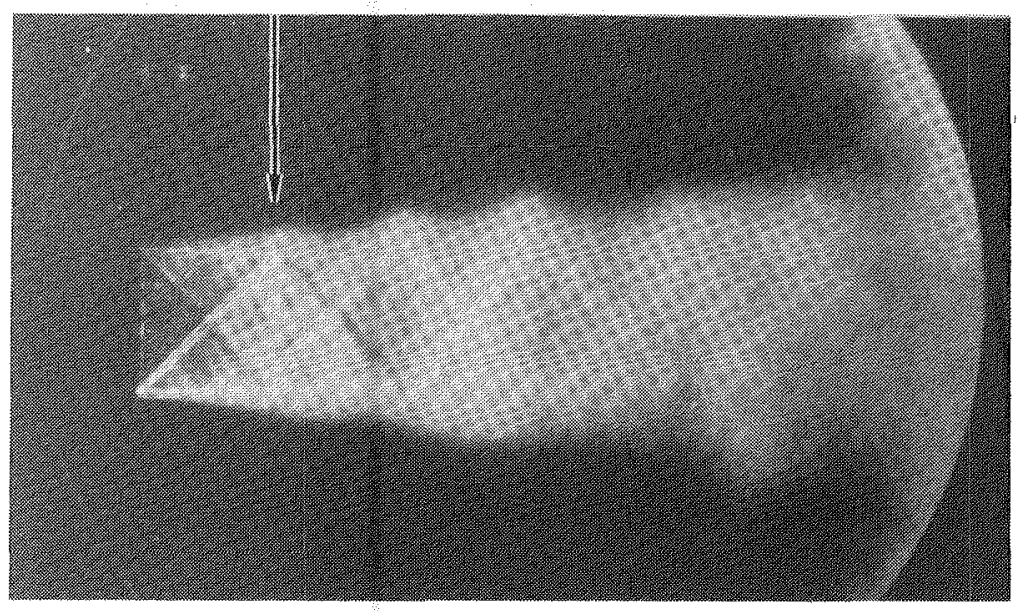

a.

$\phi=0 \mathrm{deg}$.

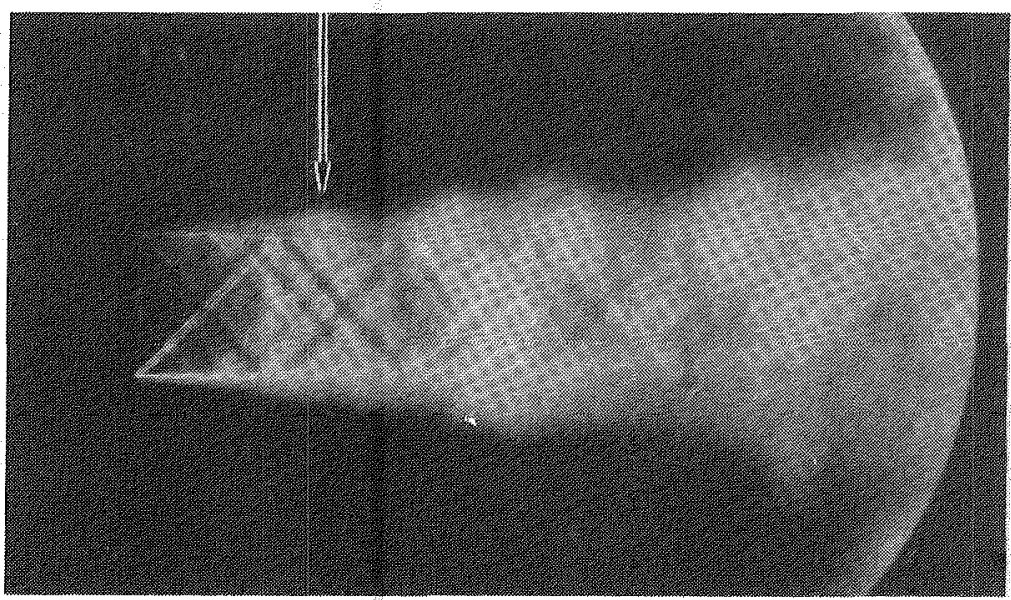

b.

$\phi=60 \mathrm{deg}$.

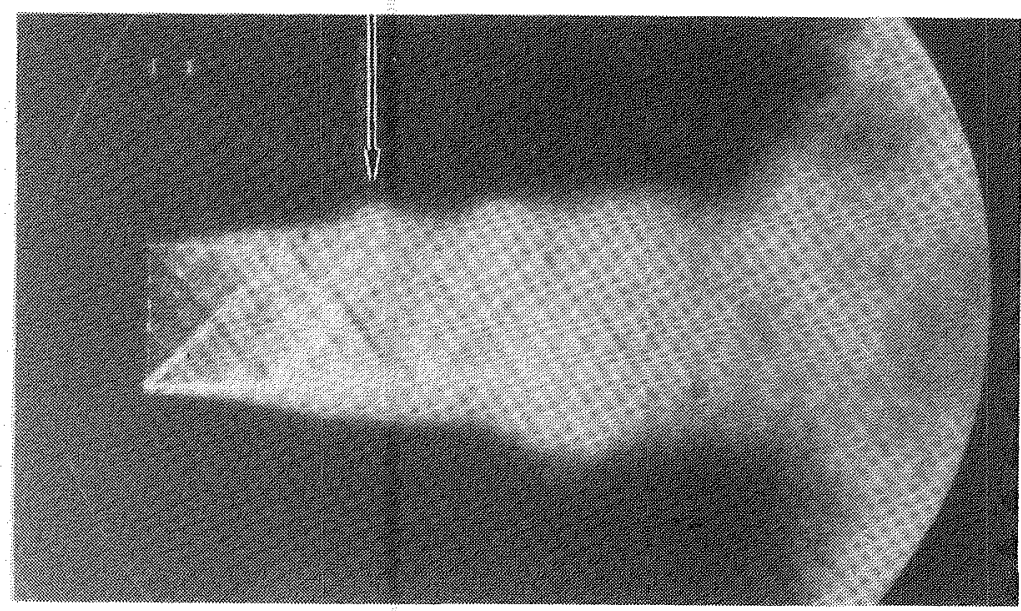

c.

$\phi=120 \mathrm{deg}$.

Figure 3.18 a-c. Ensemble-averaged photograph of an excited jet.

(Knife edge: Vertical, $\mathrm{M}_{\mathrm{j}}=1.37, \mathrm{~S}_{\mathrm{e}}=0.4, \mathrm{~V}_{\mathrm{D}}=19 \mathrm{~V}$, $\left.f_{S}=3125\right)$ 


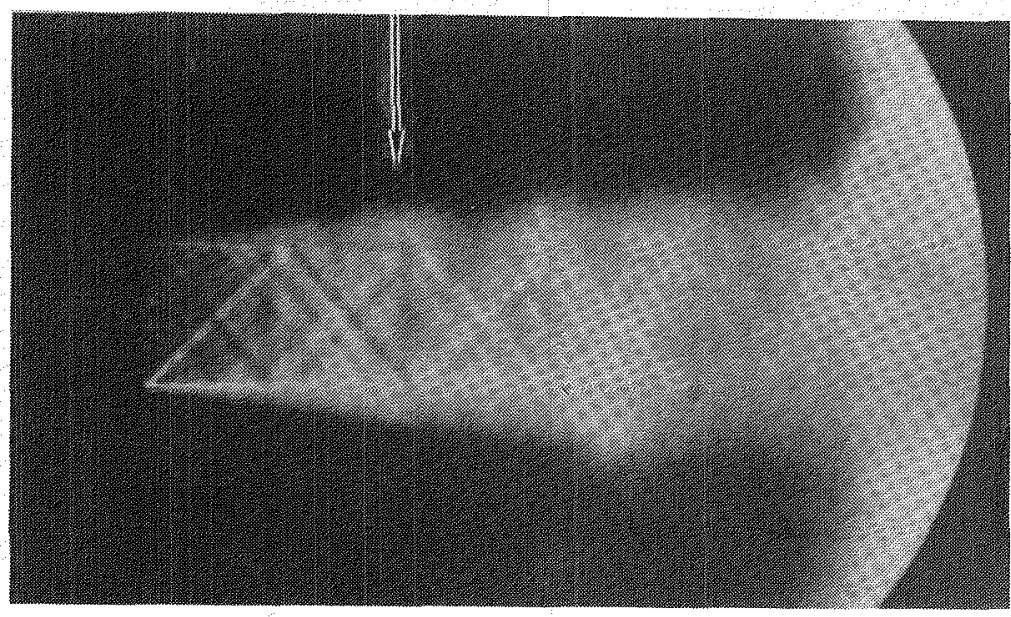

d.

$\phi=180 \mathrm{deg}$.

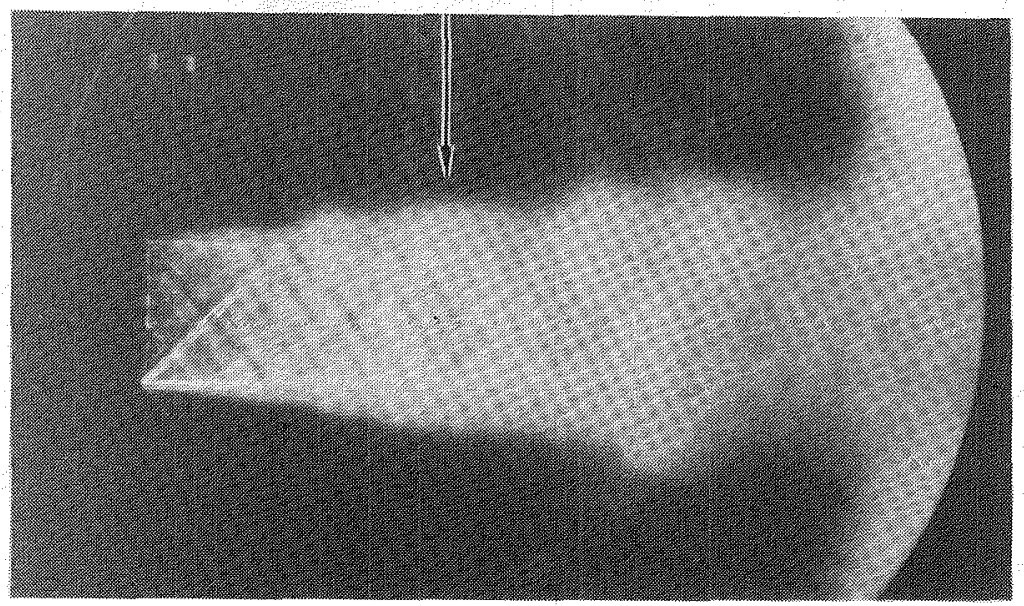

e.

$\phi=240 \mathrm{deg}$

Figure 3.18 d-e. Ensemble-averaged photograph of an excited jet.

(Knife edge: Vertical, $\mathrm{M}_{j}=1.37, \mathrm{~s}_{\mathrm{e}}=0.4, \mathrm{~V}_{\mathrm{D}}=19 \mathrm{~V}$, $\left.f_{s}=3125 \mathrm{~Hz}\right)$ 


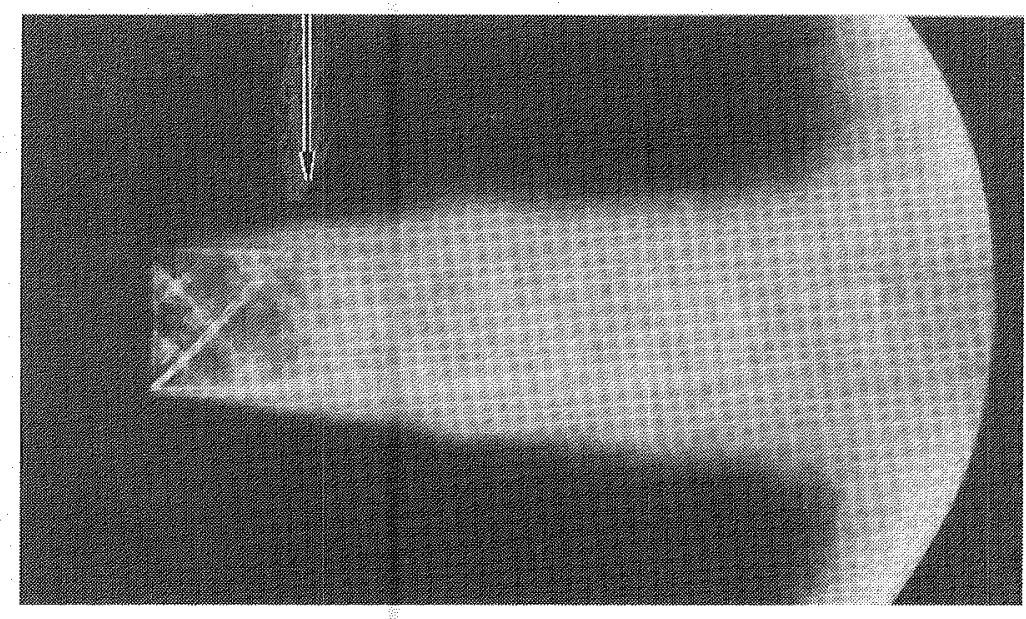

a.

$\phi=0 \mathrm{deg}$

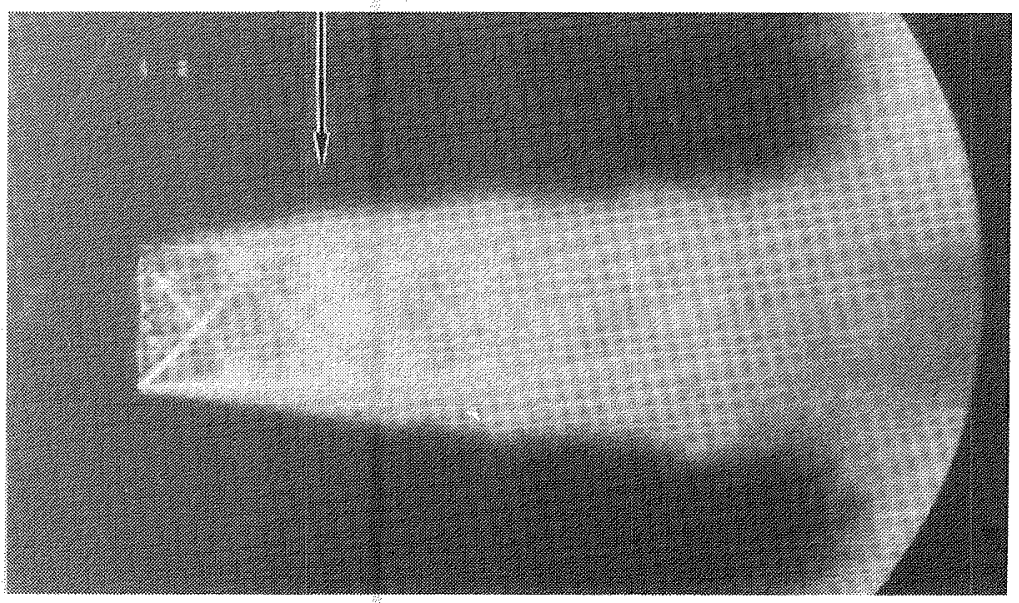

b.

$\phi=60 \mathrm{deg}$.

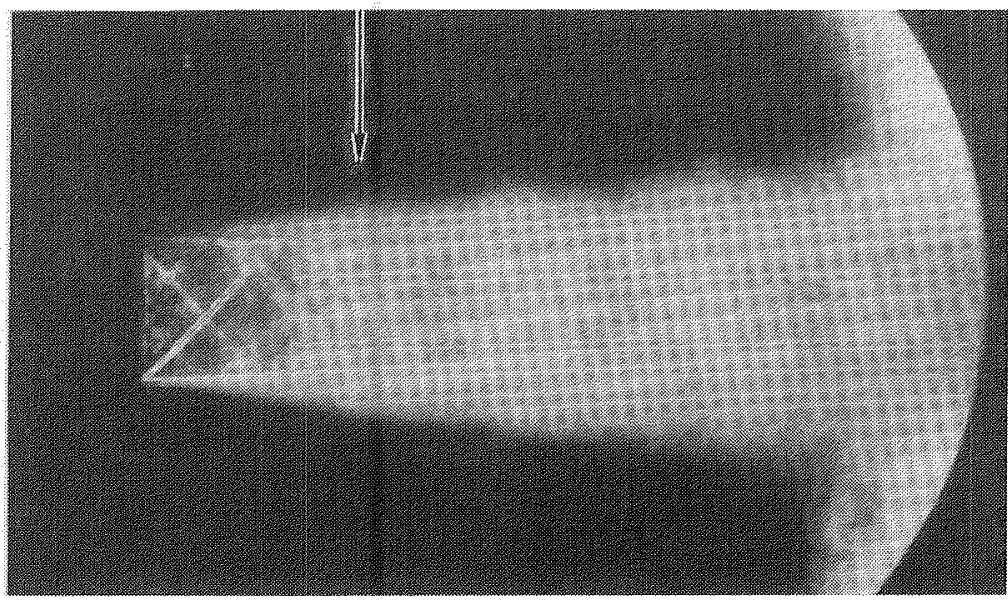

c.

$\phi=120 \mathrm{deg}$.

Figure 3.19 a-c. Ensemble-averaged photograph of an excited jet.

(Knife edge: Vertica1, $\mathrm{M}_{j}=1.37, \mathrm{~S}_{\mathrm{e}}=0.6, \mathrm{~V}_{\mathrm{D}}=19 \mathrm{~V}$, $\left.\mathrm{f}_{\mathrm{S}}=4660 \mathrm{~Hz}\right)$ 


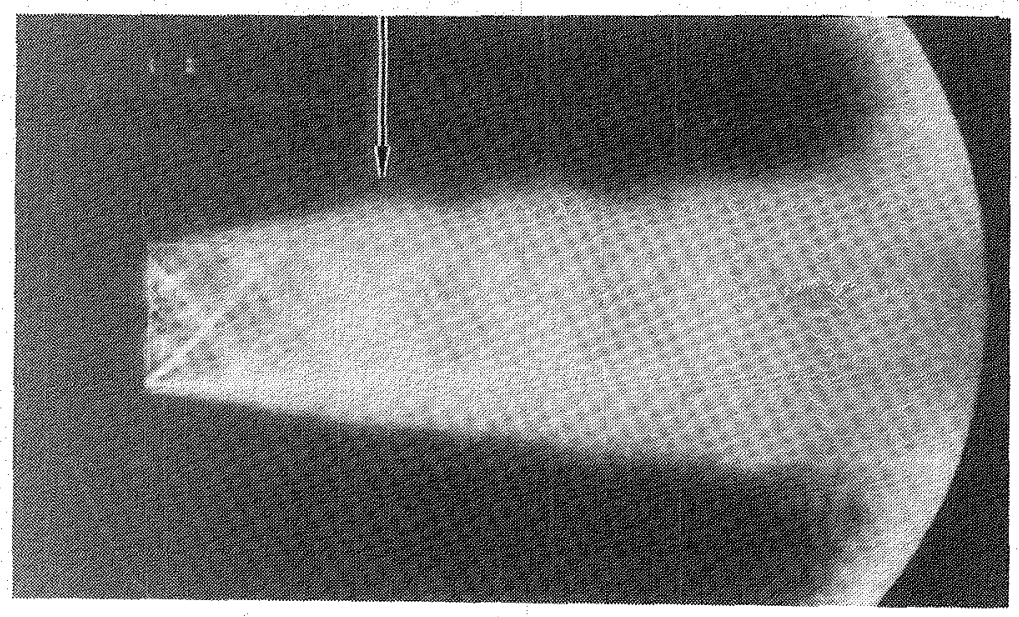

d.

$\phi=180 \mathrm{deg}$.

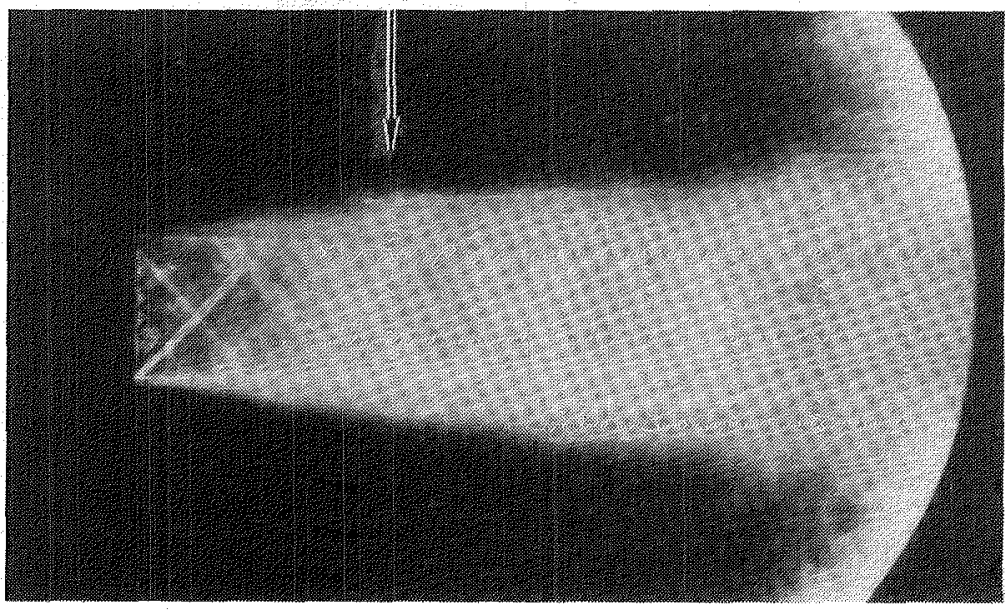

e.

$\phi=240 \mathrm{deg}$.

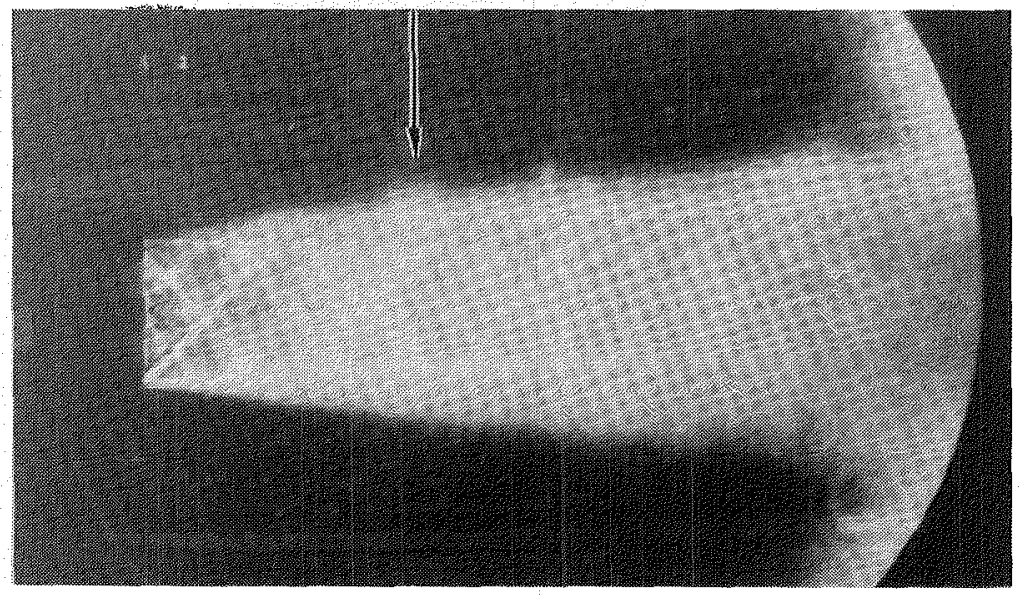

f.

$\phi=300 \mathrm{deg}$.

Figure $3.19 \mathrm{~d}-\mathrm{f}$. Ensemble-averaged photograph of an excited jet.

(Knife edge: Vertical, $\mathrm{M}_{\mathrm{j}}=1.37, \mathrm{~S}_{\mathrm{e}}=0.6, \mathrm{~V}_{\mathrm{D}}=19 \mathrm{~V}$, $\mathrm{f}_{\mathrm{S}}=4660 \mathrm{~Hz}$ ) 


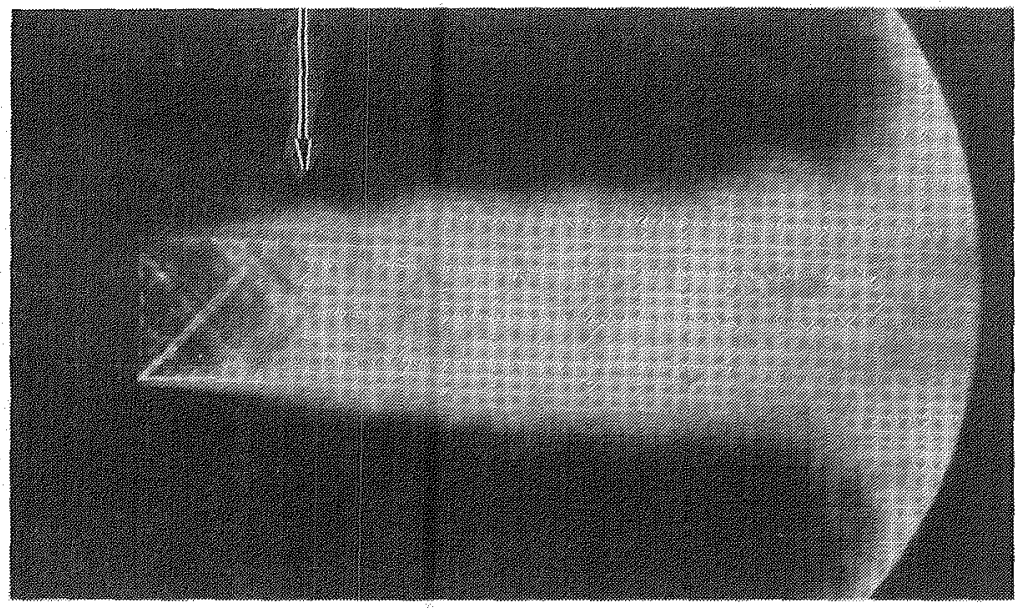

a.

$\phi=0 \mathrm{deg}$.

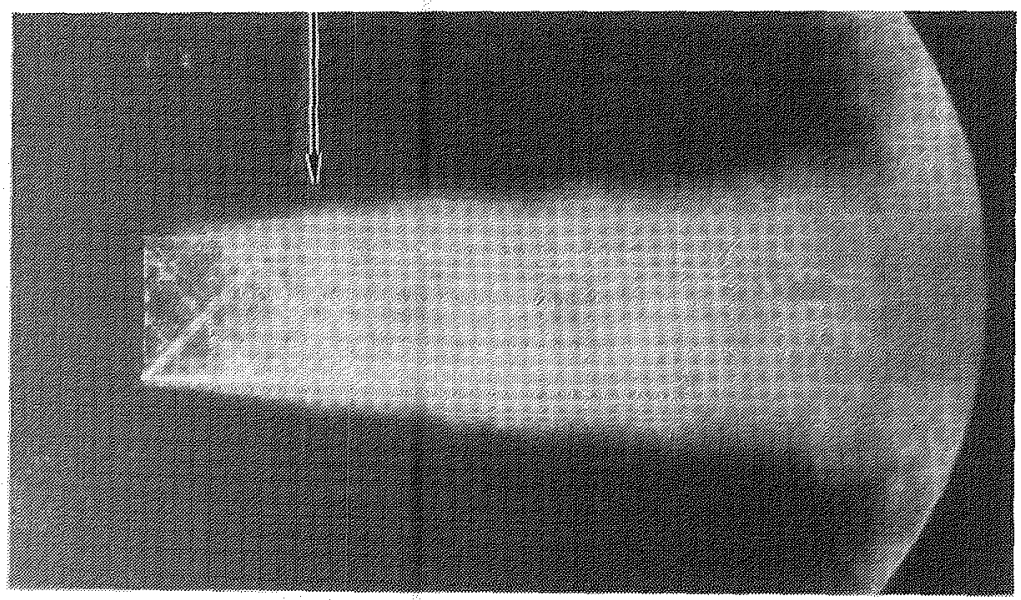

b.

$\phi=60 \mathrm{deg}$.

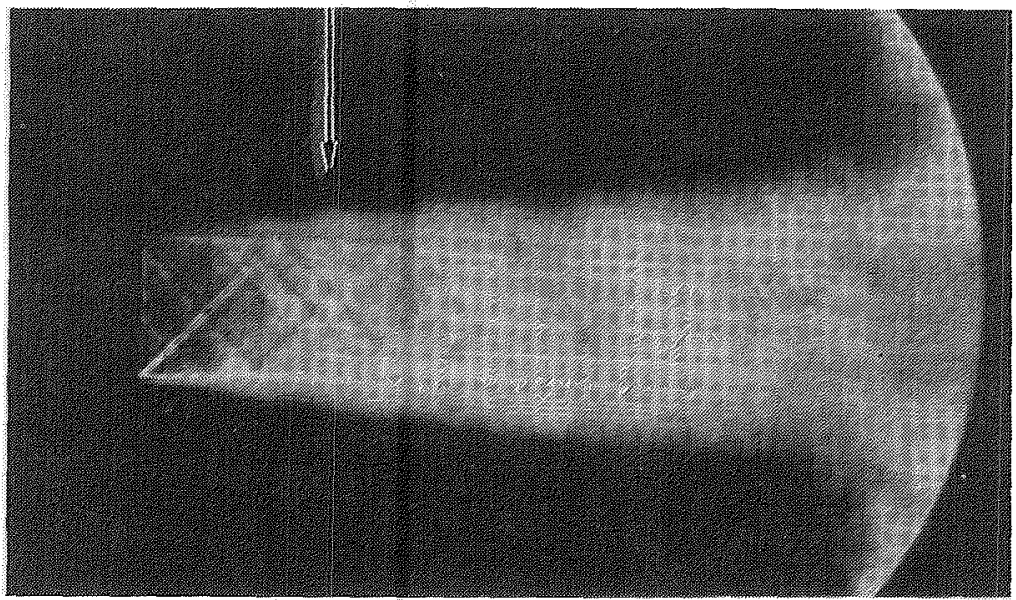

c.

$\phi=120 \mathrm{deg}$.

Figure 3.20 a-c. Ensemble-averaged photograph of an excited jet.

(Knife edge: Vertical, $\mathrm{M}_{j}=1.37, \mathrm{~S}_{\mathrm{e}}=0.7, \mathrm{~V}_{\mathrm{D}}=19 \mathrm{~V}$,
$\left.\mathrm{f}_{\mathrm{s}}=5440 \mathrm{~Hz}\right)$ 


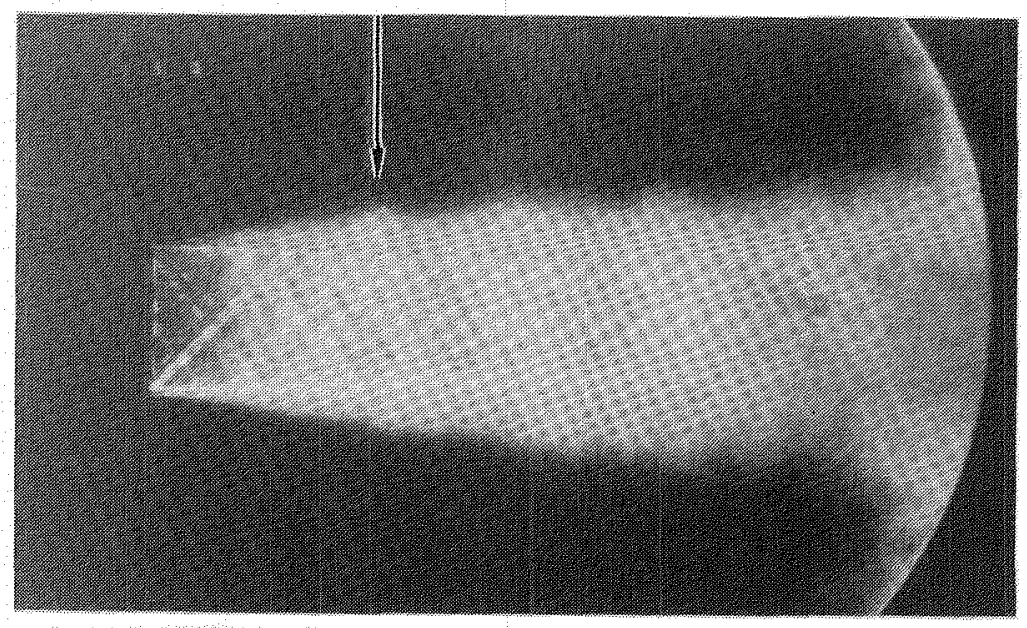

d.

$\phi=180 \mathrm{deg}$.

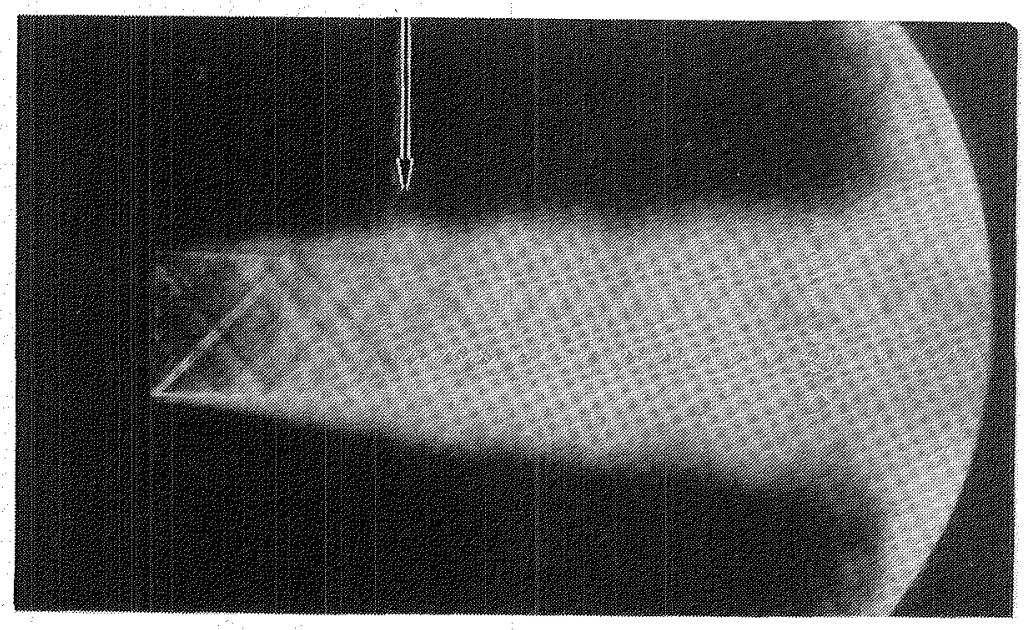

e.

$\phi=240 \mathrm{deg}$.

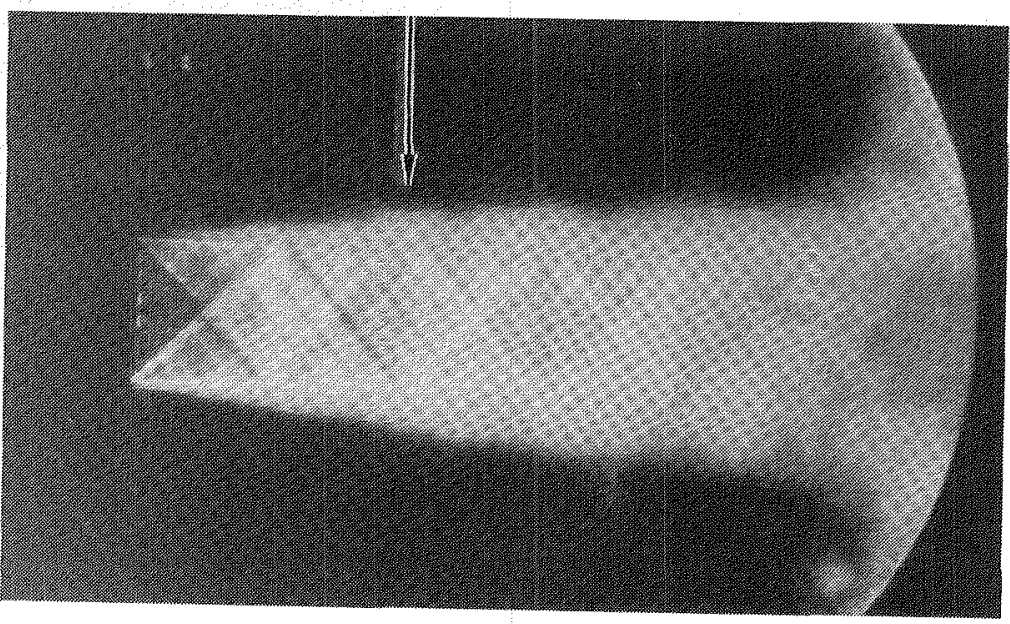

f.

$\phi=300 \mathrm{deg}$.

Figure 3.20 d-f. Ensemble-averaged photograph of an excited jet. (Knife edge: Vertical, $\mathrm{M}_{j}=1.37, \mathrm{~S}_{\mathrm{e}}=0.7, \mathrm{~V}_{\mathrm{D}}=19 \mathrm{~V}$,
$\mathrm{f}_{\mathrm{S}}=5440 \mathrm{~Hz}$ ) 


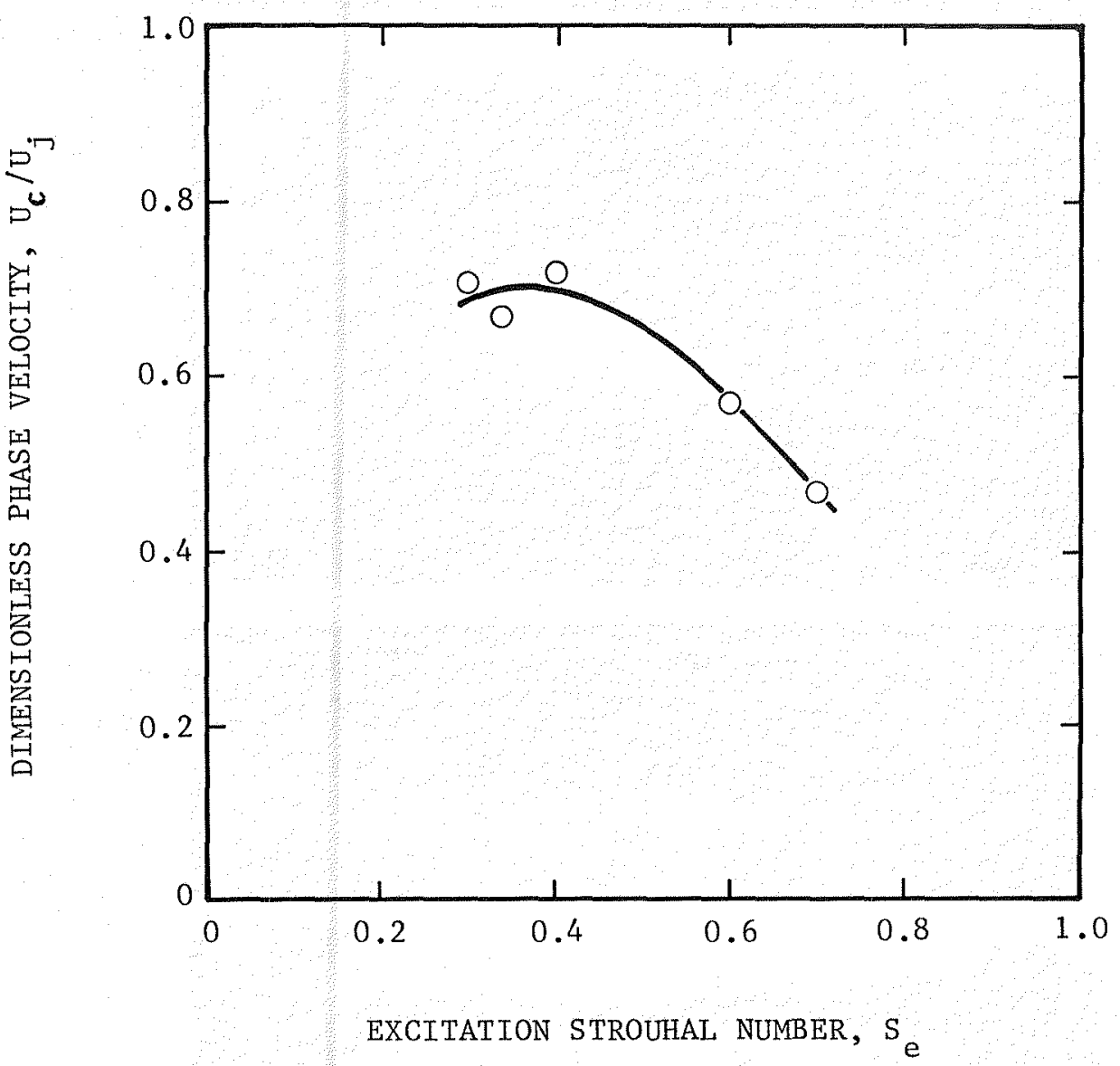

Figure 3.21 Variation of large-scale structure phase velocity with excitation Strouhal number 
function of the jet excitation Strouhal number. It appears to be a function of Strouhal number.

\subsection{Initia1 Conclusions}

The purpose of the flow visualization task was to demonstrate the existence of the large-scale structures in fully expanded, supersonic, high Reynolds number jets. Furthermore, it was expected that the visualization study would provide sufficient information about the preferential frequencies of the large-scale instability in supersonic jets to determine the most favorable experimental conditions for flow measurements under Task 2 of this program.

The following conclusions may be drawn from the results of the "Flow Visualization" task:

1. The large-scale structures can be excited in fully expanded, supersonic, high Reynolds number jets, and they prevail even beyond the potential core of the jet.

2. The most preferential excitation strouhal number for these largescale structures is in the vicinity of 0.4 for this particular fully expanded Mach number.

3. The normalized phase velocity of the large-scale structure is 0.7 for the case of the preferred excitation strouhal number of 0.4 . However, it appears that the phase velocity decreases for the excitation Strouhal numbers higher than the preferred one.

Based on the above-stated conclusions, it was decided to conduct the quantitative laser-velocimeter flow measurements at the excitation Strouhal number of 0.4 and the excitation voltage of $19 \mathrm{~V}$. The results of LV measurements are described in the following section. 


\subsection{QUANTITATIVE MEASUREMENTS}

Quantitative flow velocity measurements using laser velocimeter are described in this section. These measurements were intended as a quantitative supplement to the qualitative results obtained under the flow visualization task. However, the LV measurements could not be completed in the full extent as intended because of a number of instrument-related problems including unexpectedly low signal-to-noise ratio of the LV signal at various locations in the jet plume of a supersonic jet. Background of these difficulties and the lessons learned therefrom are given in Section 5.0.

This section starts out with the results of jet-noise spectra measurements made to verify the isothermal jet operating conditions for which almost shockless expansion occurs. The results of mean velocity and mean turbulence intensity measurements along the jet centerline and along jet radials at four axial locations are then presented. This is followed by a description of the results of conditionally sampled LV measurements. Comparisons of the present experimental data with other existing measurements are shown in the fourth subsection. Finally, the conclusions reached under the both, flow visualization and LV measurements, experimental tasks are summarized in the last subsection.

\subsection{Jet-Noise Spectra}

As shown in Appendix A, it was established from schlieren flow visualization that out of a set of three C-D nozzles available, the nozzle selected for this program had the weakest shock waves at its design Mach number. To ensure that, indeed these shockwaves were rather weak, limited jet noise measurements were made first. Noise spectra were measured by a 6.4-mmdia B\&K microphone placed at an angle of $90 \mathrm{deg}$, with respect to the jet axis. The radial distance of the microphone from the nozzle axis was $R=50 \mathrm{~mm}$, and the axial distance from the nozzle exit plane was $X=15 \mathrm{~mm}$.

These spectra were measured for different operating conditions. Three of these spectra are shown in Figures 4.1 through 4.3. The peak sound pressure levels, derived from the spectra measurements, were plotted as a function of the jet pressure ratio and are shown in Figure 4.4. Although the spectra measurements were not carried out in an anechoic chamber, it was assumed that the spectrum at $=3.10$ (Figure 4.2) was that for the supposedly a "fully expanded" jet, since it contained the smallest contribution of screech-related discrete tones. The corresponding Mach number for the fully expanded jet is $M_{j}=1.38$. This is in agreement with the findings from the flow visualization experiments, described in Appendix A.

\subsection{Mean Flow Measurements}

\section{Global Features of the Flow not Affected by Mild Acoustic Excitation}

Laser velocimeter measurements of mean velocity and turbulence intensity were made for both unexcited and excited jets. (The term "excited" is used loosely here to denote the condition when discrete tone sound was imposed upon 


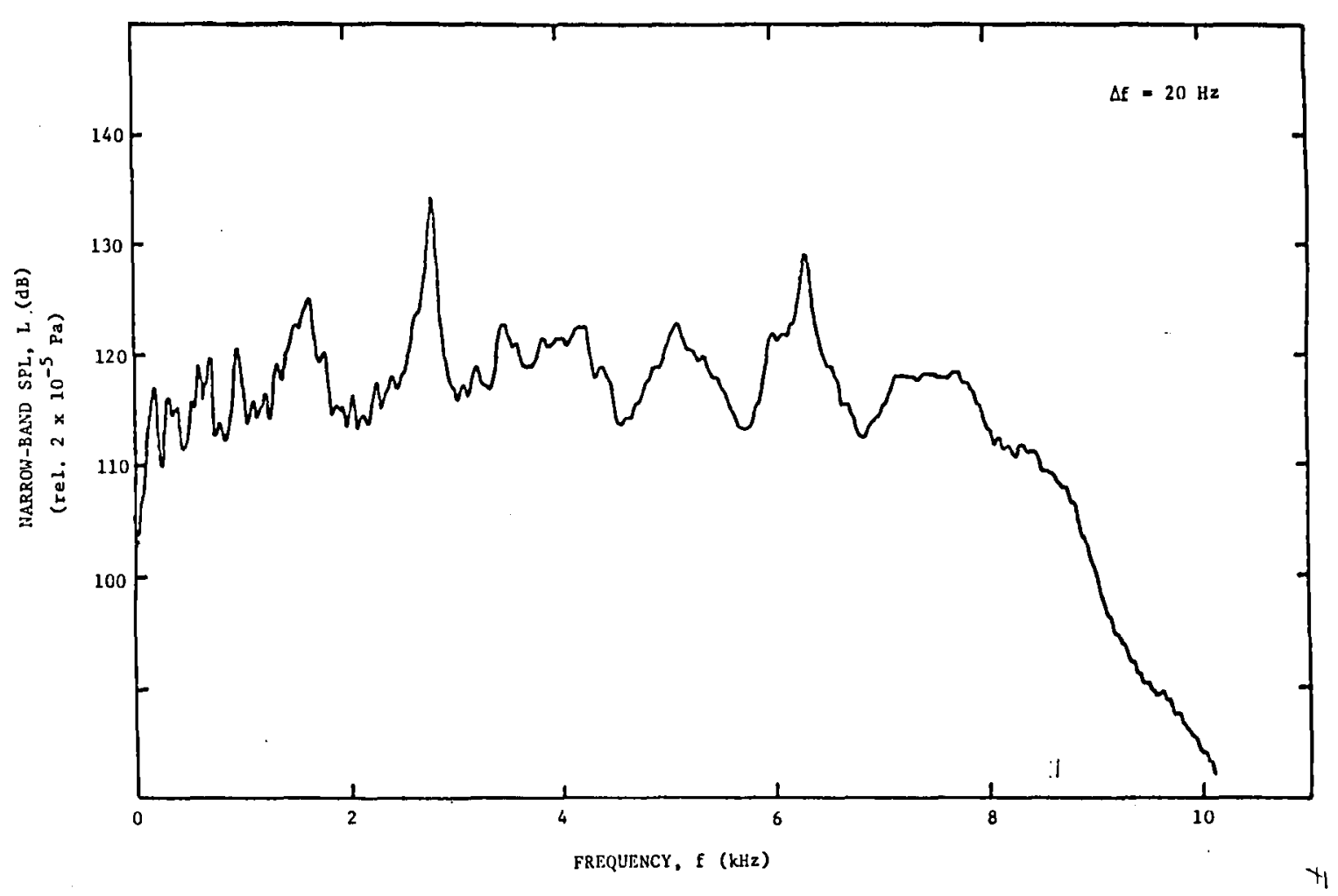

Figure 4.1 Jet-noise frequency spectrum for $\xi=2.99$. 


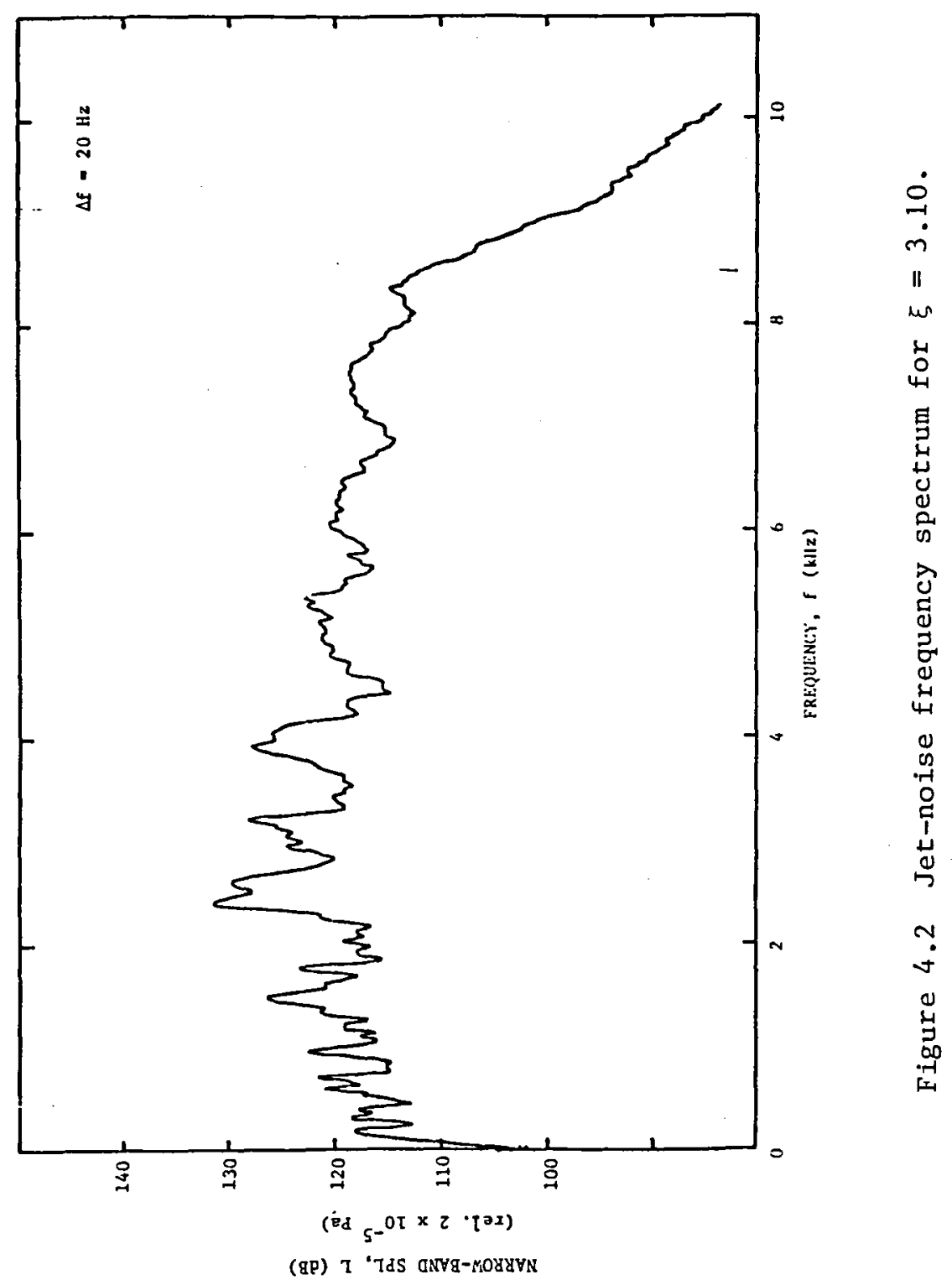




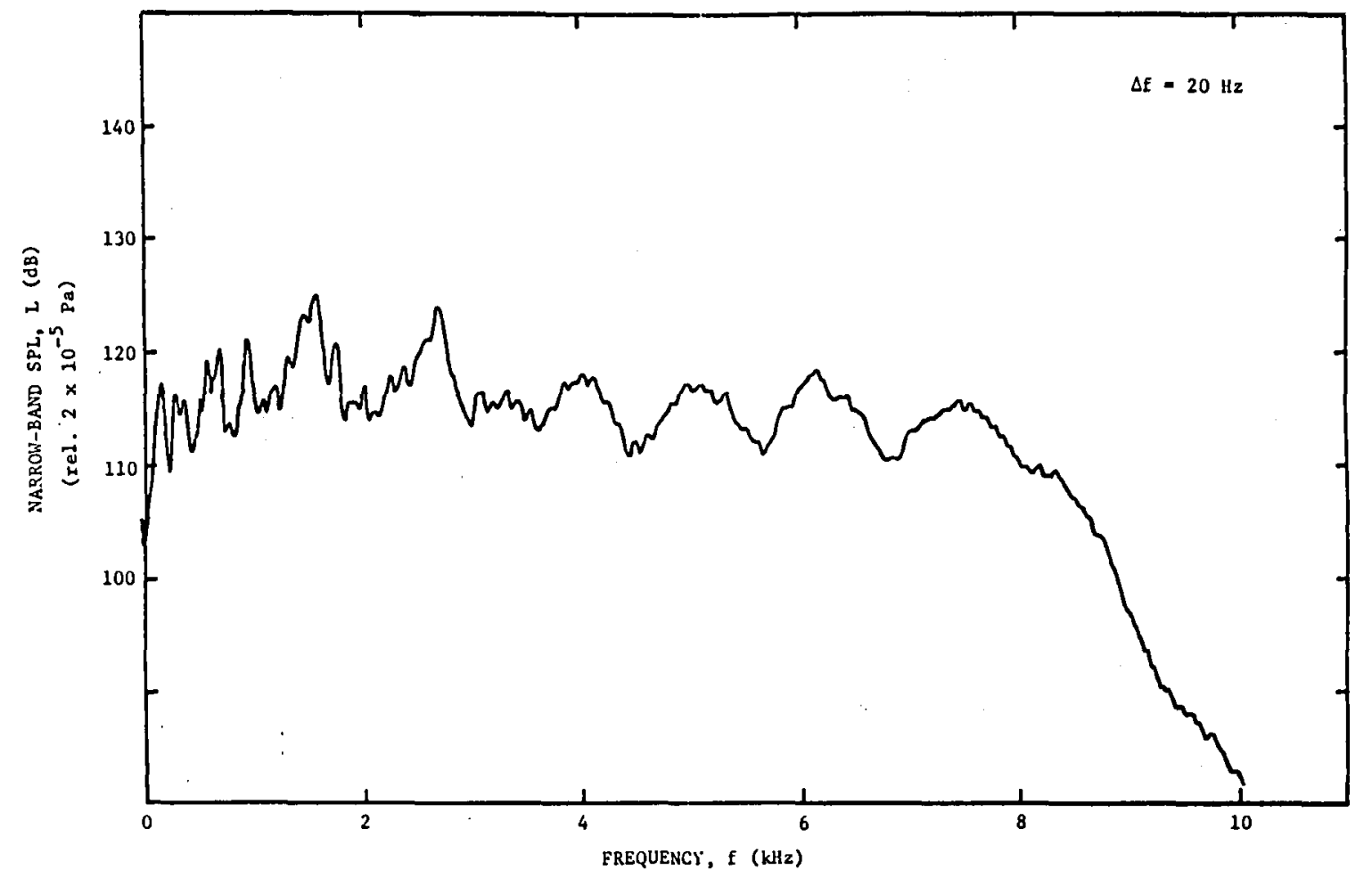

Figure 4.3 Jet-noise frequency spectrum for $\xi=3.42$. 


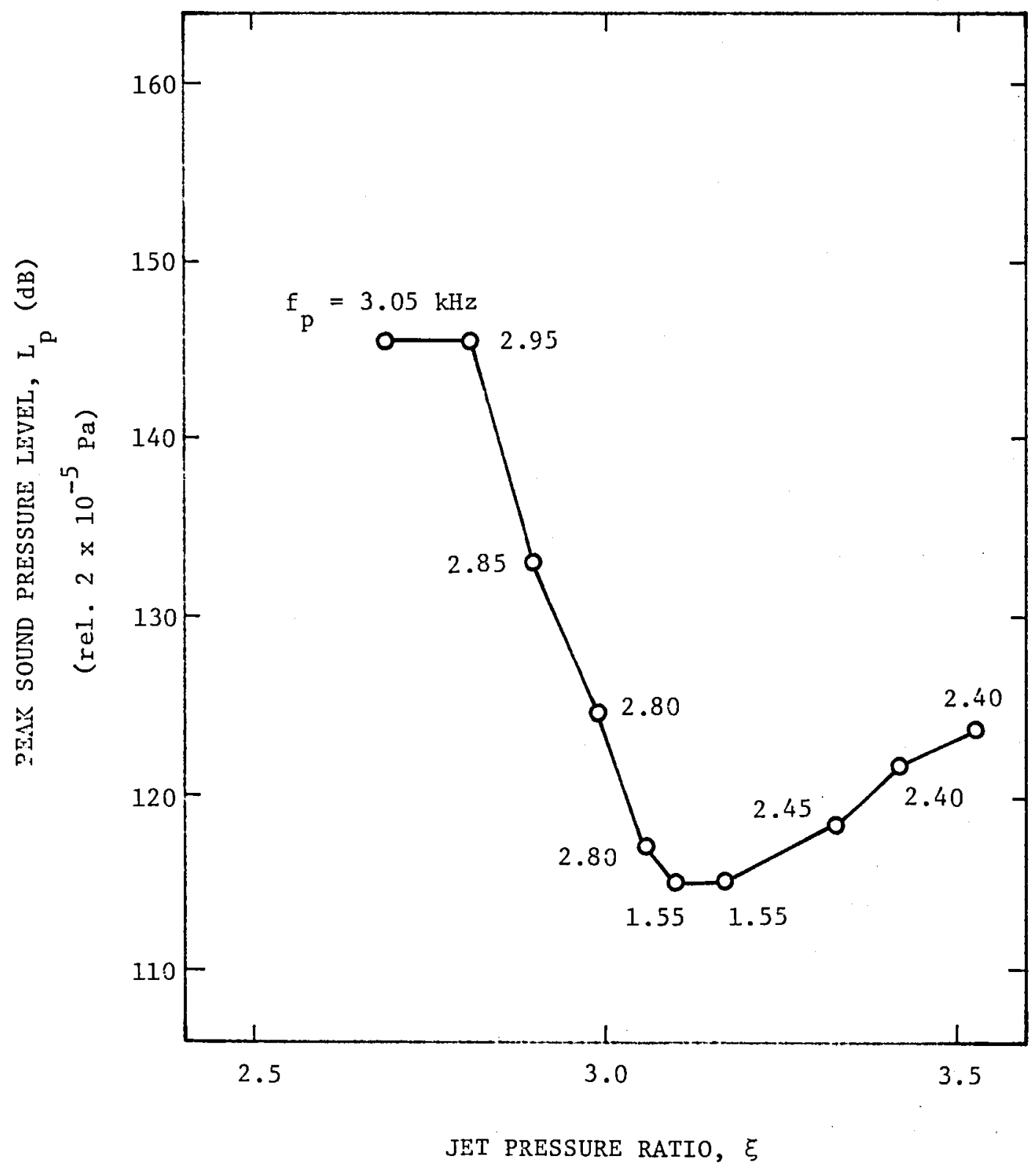

Figure 4.4 Peak sound pressure level variation with jet pressure ratio. 
the jet.) Schlieren pictures of the fully expanded jet of $M_{j}=1.37$, described in the previous section, showed that the most effective excitation Strouhal number is 0.4 . Thus, the laser velocimeter experiments were made at this excitation Strouhal number and at the excitation voltage equal to that used in the flow visualization experiments. Total temperature of the flow was kept at $370 \mathrm{~K}$ to operate the jet at isothermal conditions.

The experiments started out with the measurement of radial velocity profiles. These profiles were measured along two radii: vertical (axis $z$ ) and horizontal (axis $Y$ ). The profiles along the vertical radius were measured for both excited and unexcited jets at axial locations of $X / D=3,6$, and 9 ; whereas, the profiles along the horizontal radius were measured only for the unexcited jet at axial locations of $X / D=6,9$, and 15 . The profiles along vertical radii are shown in Figures 4.5 through 4.7 ; the profiles along horizontal radii are shown in Figures 4.8 through 4.10 . It should be mentioned that both the local velocity and turbulence intensity measured by the laser velocimeter are normalized by the jet exit velocity computed from the measured plenum total pressure and total temperature and from the ambient pressure.

As seen in Figures 4.5 through 4.10, there is no difference between the excited and unexcited jet mean velocity and turbulence intensity profiles. Thus, the excitation tone, which serves as a triggering signal for acquiring conditionally sampled laser velocimeter data, does not measurably alter the mean velocity and turbulence intensity characteristics of the jet itself. This indicates that, although visible in the flow visualization experiments, the excited large-scale structure in the jet shear layer is rather weak in amplitude and does not affect the flow structure globally.

\section{Velocity Profiles Found Nonaxisymetric}

Closer examination of Figures 4.5 through 4.10 shows that the velocity and turbulence intensity profiles are not exactly axisymetric. This small distortion was observed regardless of the excitation condition, indicating that it is probably not caused by the conditions upstream of the test nozzle. One of the reasons for this distortion may be the possible nonsymetry of the entrained flow distribution in the test room. To verify the extent of this distortion two additional velocity radial profiles were measured at $X / D=3$. These profiles are shown in Figures 4.11 and 4.12 .

The velocity profiles, measured at $x / D=3$ (Figures $4.5,4.11$, and 4.12) were used to reconstruct velocity contours for $100 \%, 75 \%$, and $50 \%$ of the jet exit velocity. These contours together with a nozzle 1 ip contour are shown in Figure 4.13. As seen in this figure, the jet flowfield tends to become elliptical with its major axis in the vertical direction.

\section{Axial Distribution of Mean, Half, and Turbulent Velocity Determined}

The acquired mean velocity profiles were used to determine the jet centerline and the location of half velocity points, as shown in Figure 4.14. The centerline distribution of axial mean velocity and turbulence intensity 


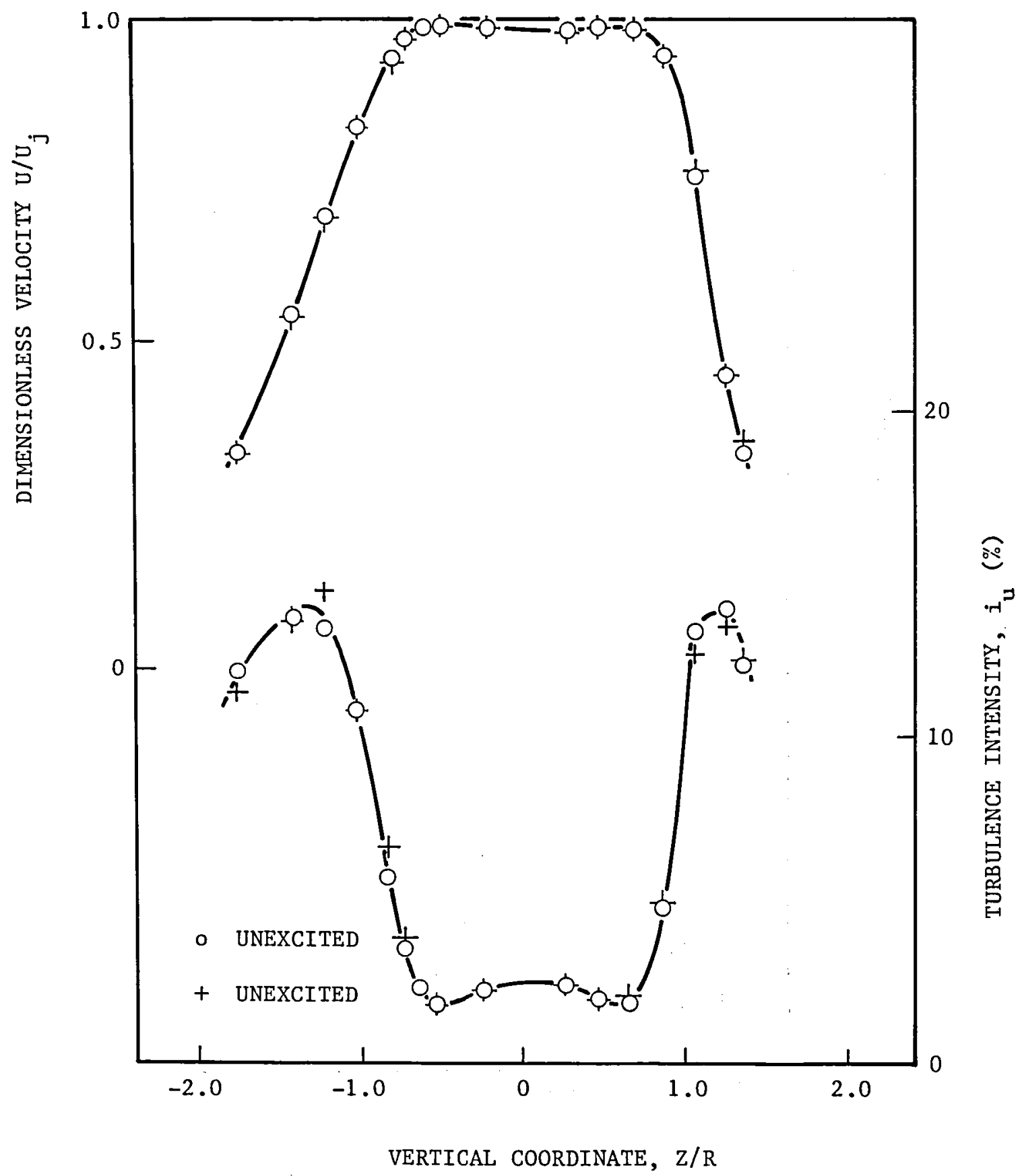

Figure 4.5 Mean velocity and turbulence intensity vertical profiles of a fully expanded, supersonic jet.

$\left(X / D=3, M_{j}=1.38, U_{j}=454 \mathrm{~m} / \mathrm{s}\right)$ 


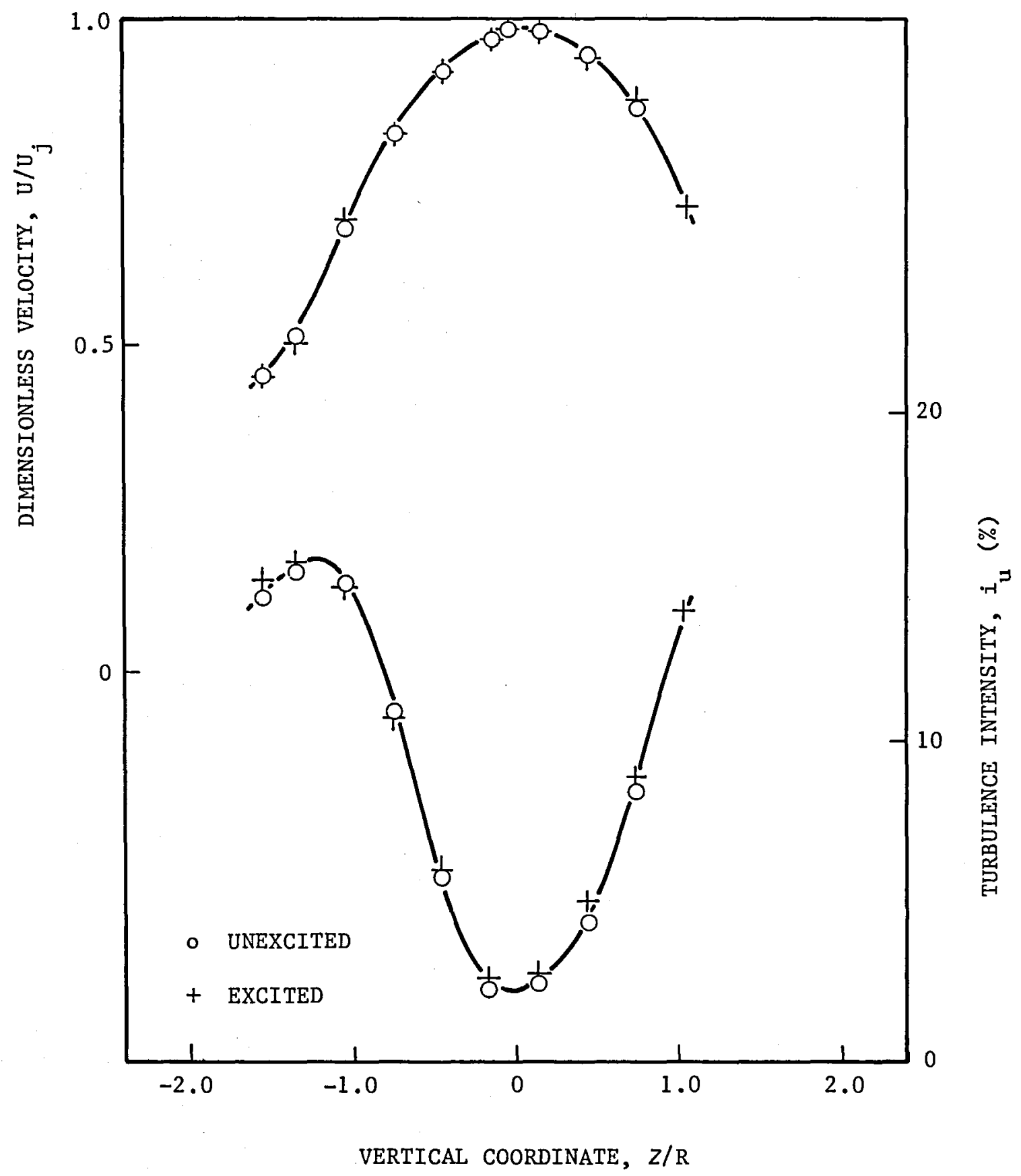

Figure 4.6. Mean velocity and turbulence intensity vertical profiles of a fully expanded, supersonic jet.

$\left(\mathrm{X} / \mathrm{D}=6, \mathrm{M}_{\mathrm{j}}=1.38, \mathrm{U}_{\mathrm{j}}=454 \mathrm{~m} / \mathrm{s}\right)$ 


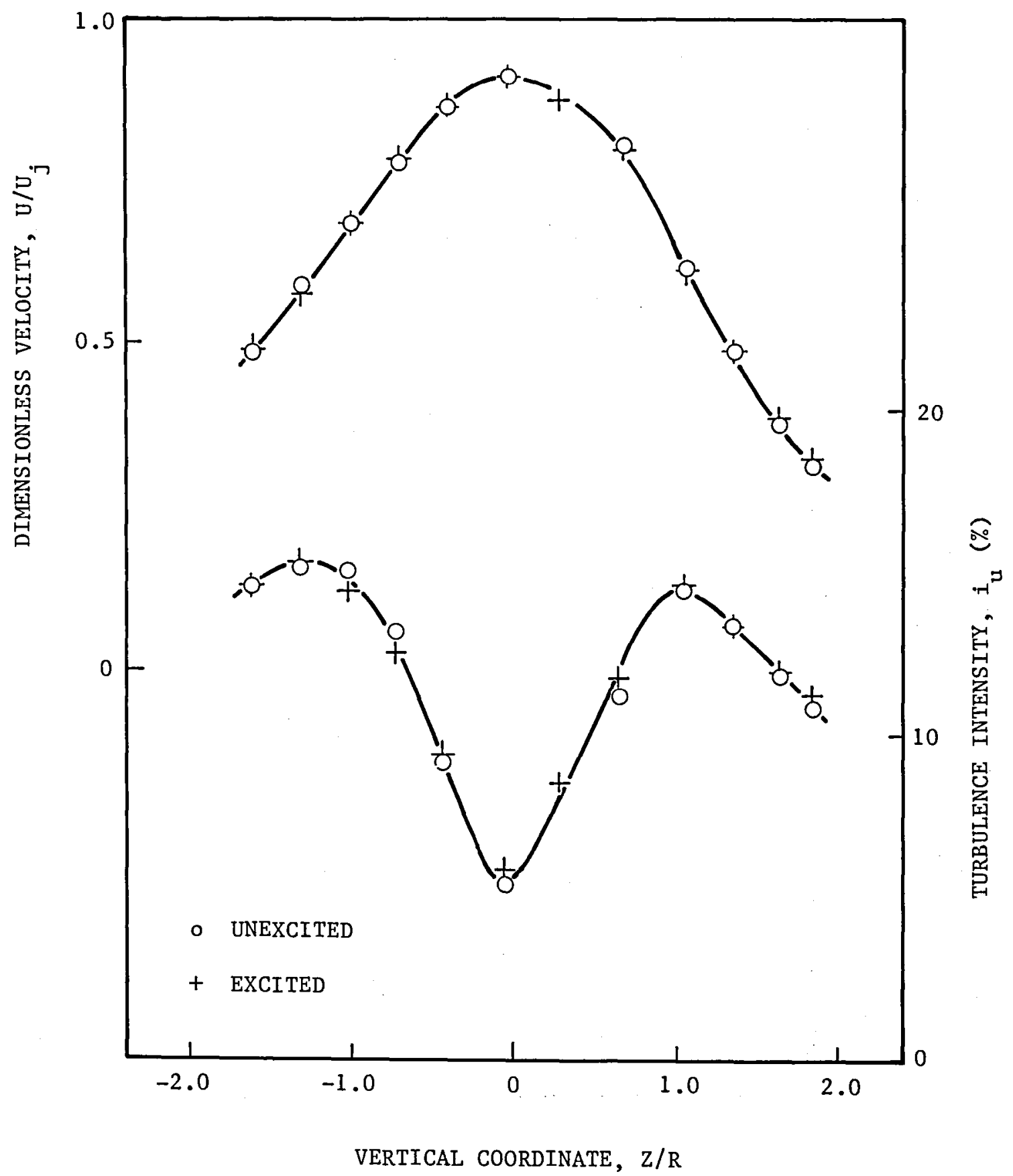

Figure 4.7. Mean velocity and turbulence intensity vertical profiles of a fu1ly expanded, supersonic jet.

$\left(\mathrm{X} / \mathrm{D}=9, \mathrm{M}_{\mathrm{j}}=1.38, \mathrm{U}_{\mathrm{j}}=455 \mathrm{~m} / \mathrm{s}\right)$ 


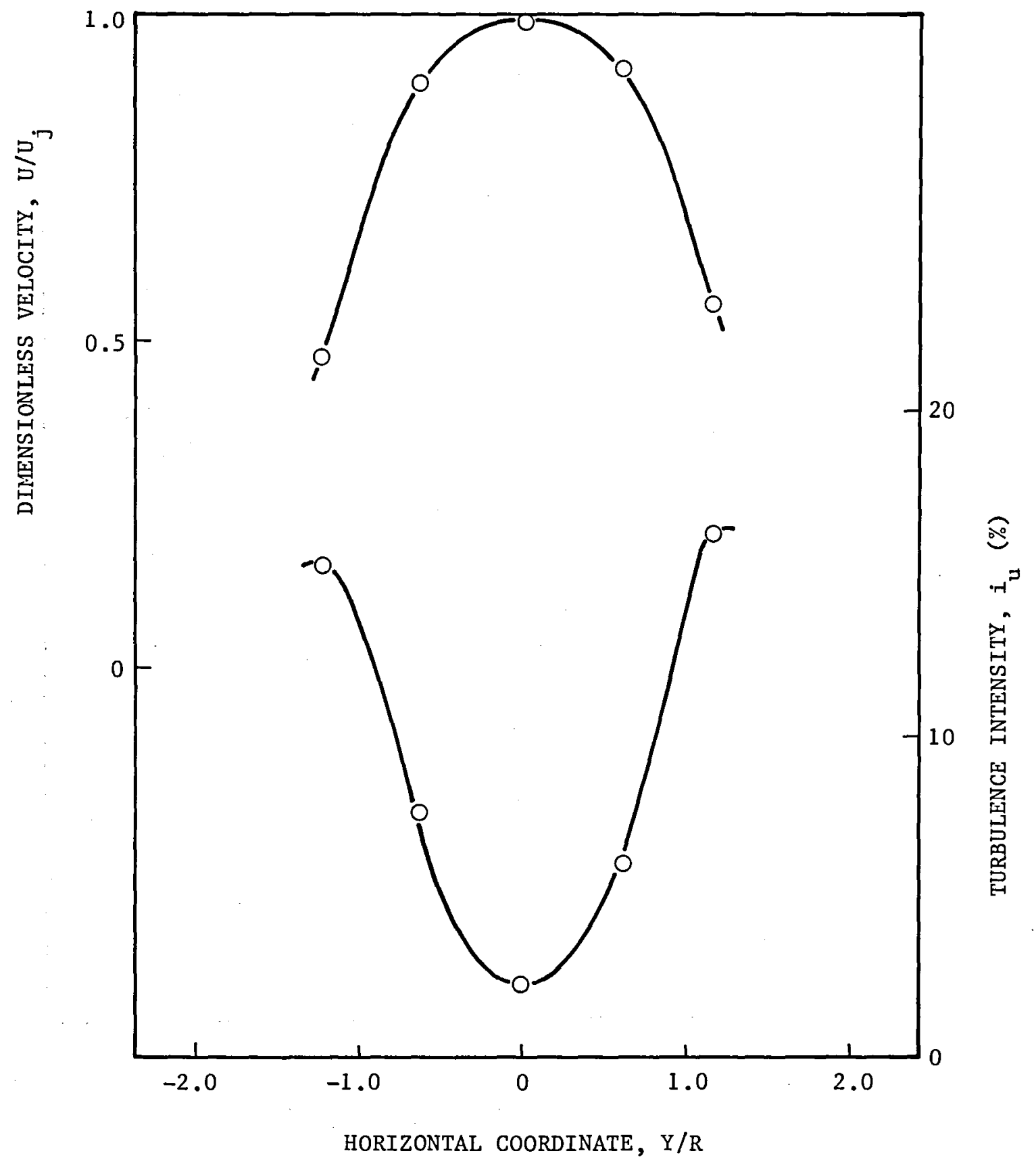

Figure 4.8. Mean velocity and turbulence intensity horizontal profiles of a fully expanded, supersonic jet.

$\left(\mathrm{X} / \mathrm{D}=6, \mathrm{M}_{\mathrm{j}}=1.38, \mathrm{U}_{\mathrm{j}}=453 \mathrm{~m} / \mathrm{s}\right)$ 


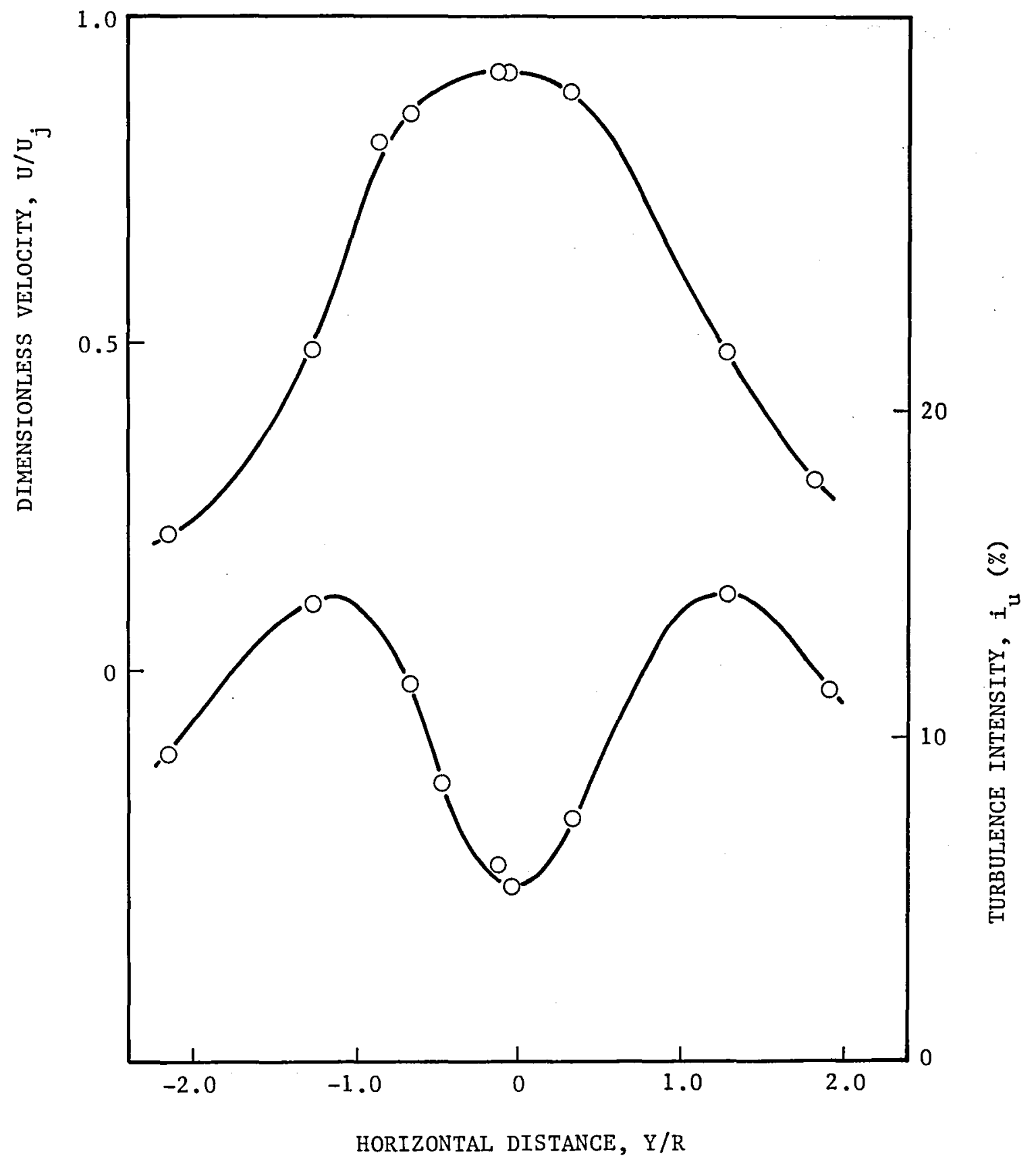

Figure 4.9. Mean velocity and turbulence intensity horizontal profiles of a fully expanded, supersonic jet. $\left(x / D=9, M_{j}=1.38, U_{j}=455 \mathrm{~m} / \mathrm{s}\right)$ 


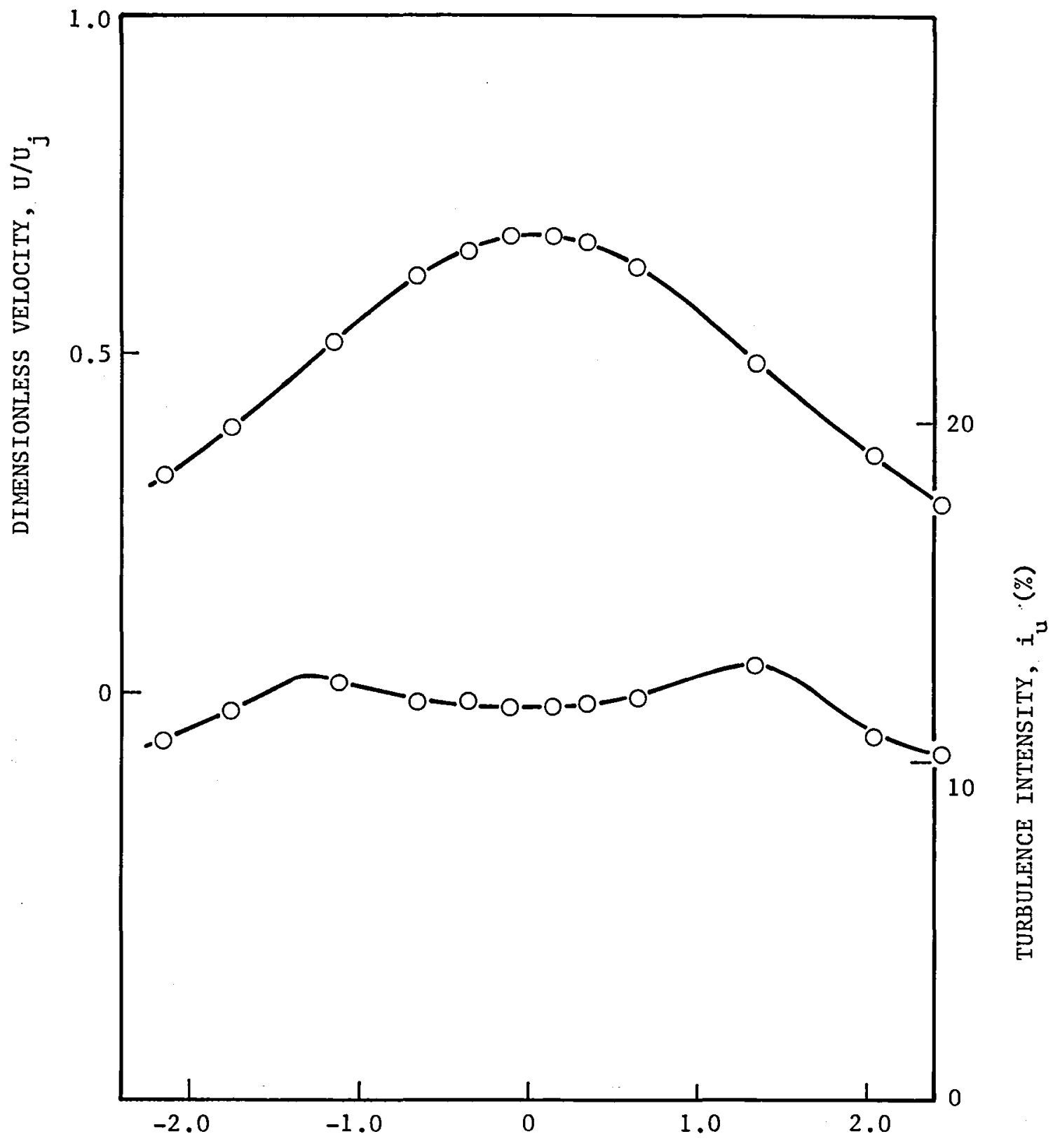

HORIZONTAL DISTANCE, $Y / R$

Figure 4.10. Mean velocity and turbulence intensity horizontal profiles of a fully expanded, supersonic jet.

$\left(X / D=15, M_{j}=1.38, U_{j}=454 \mathrm{~m} / \mathrm{s}\right)$ 


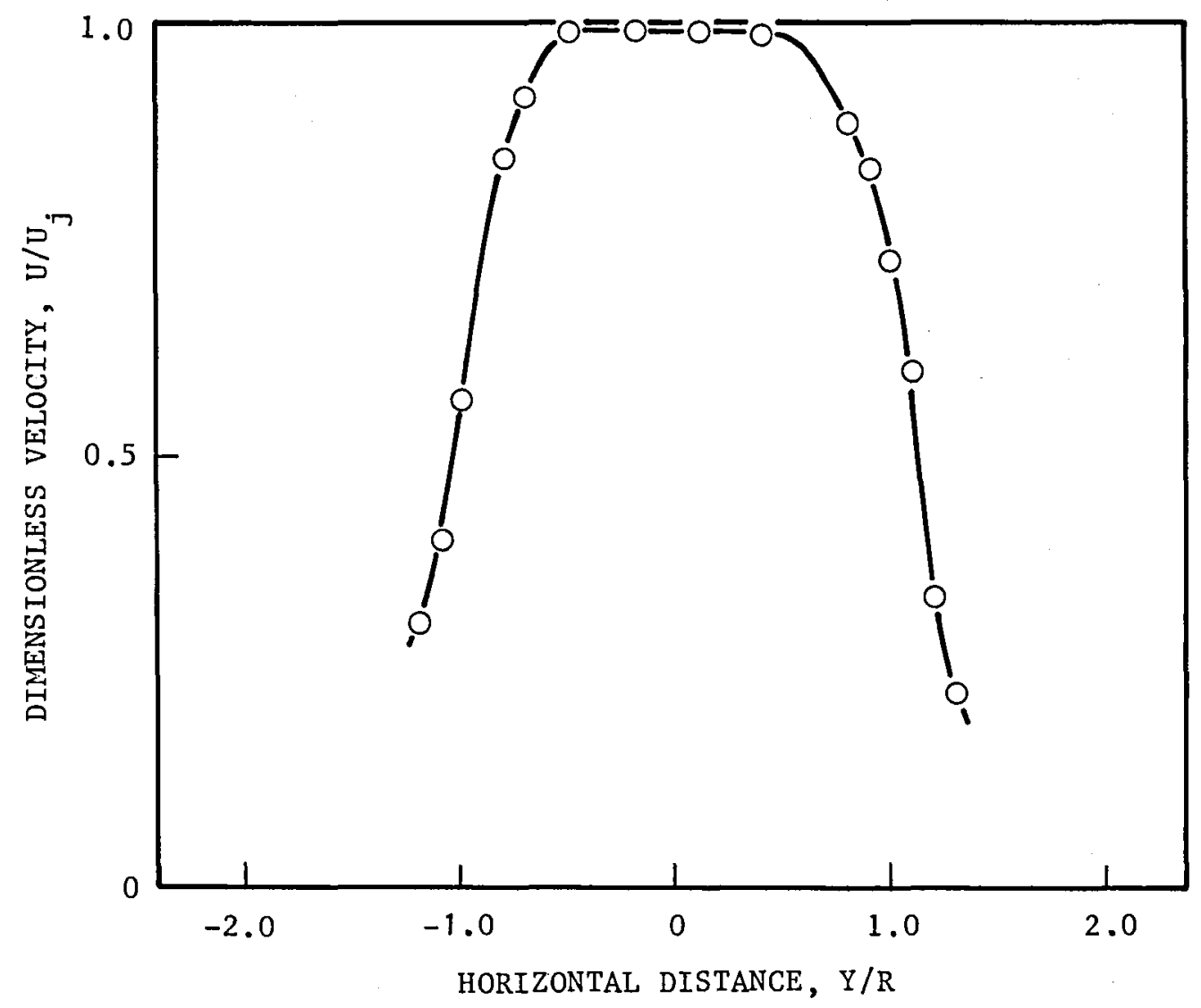

Figure 4.11 Mean velocity profile of a fully expanded supersonic jet. $\left(X / D=3, Z / R=0.1, M_{j}=1.38, U_{j}=454 \mathrm{~m} / \mathrm{s}\right)$ 


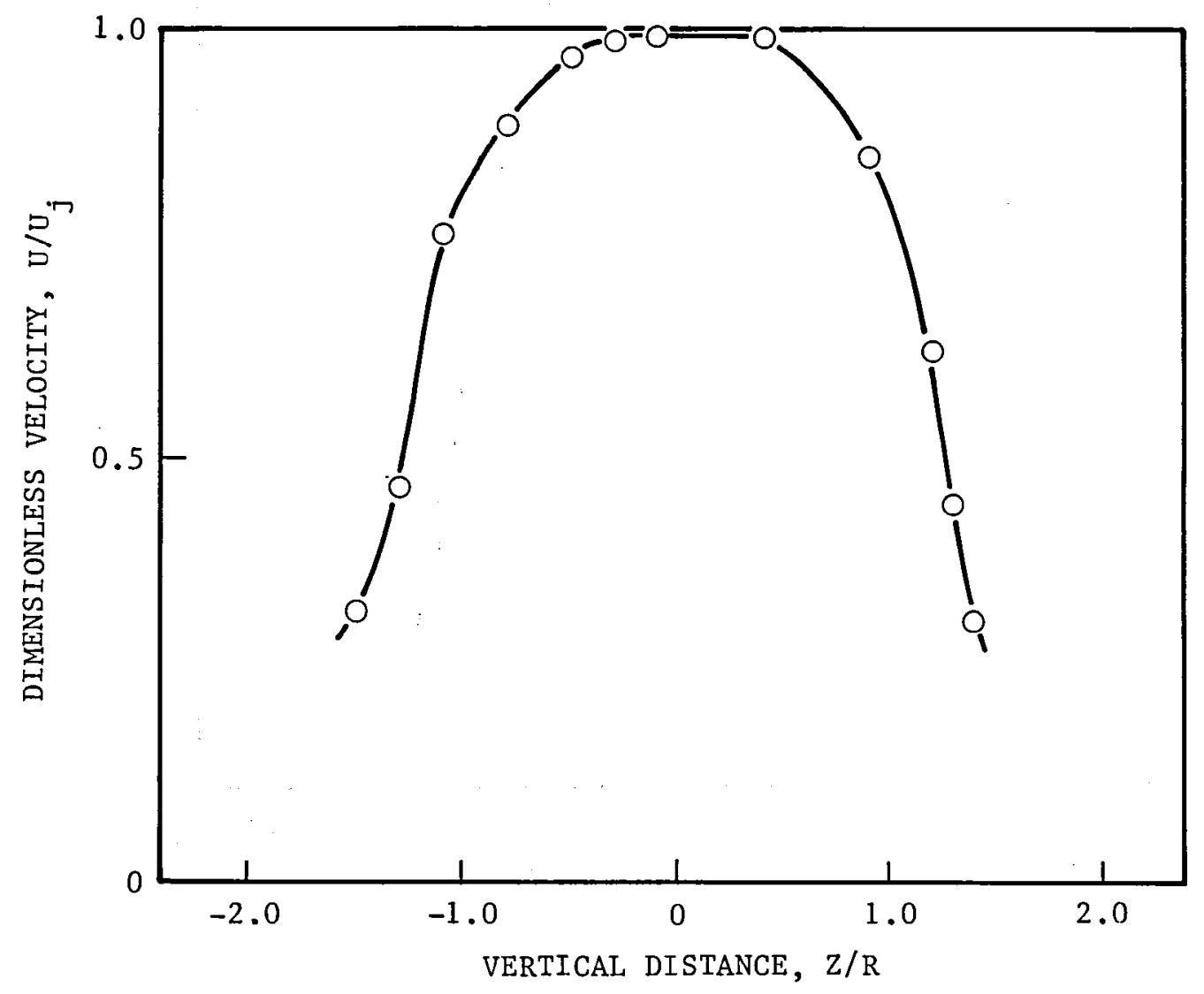

Figure 4.12 Mean velocity profile of a fully expanded supersonic jet. $\left(\mathrm{X} / \mathrm{D}=3, \mathrm{Y} / \mathrm{R}=0.34, \mathrm{M}_{\mathrm{j}}=1.38, \mathrm{U}_{\mathrm{j}}=454 \mathrm{~m} / \mathrm{s}\right)$ 


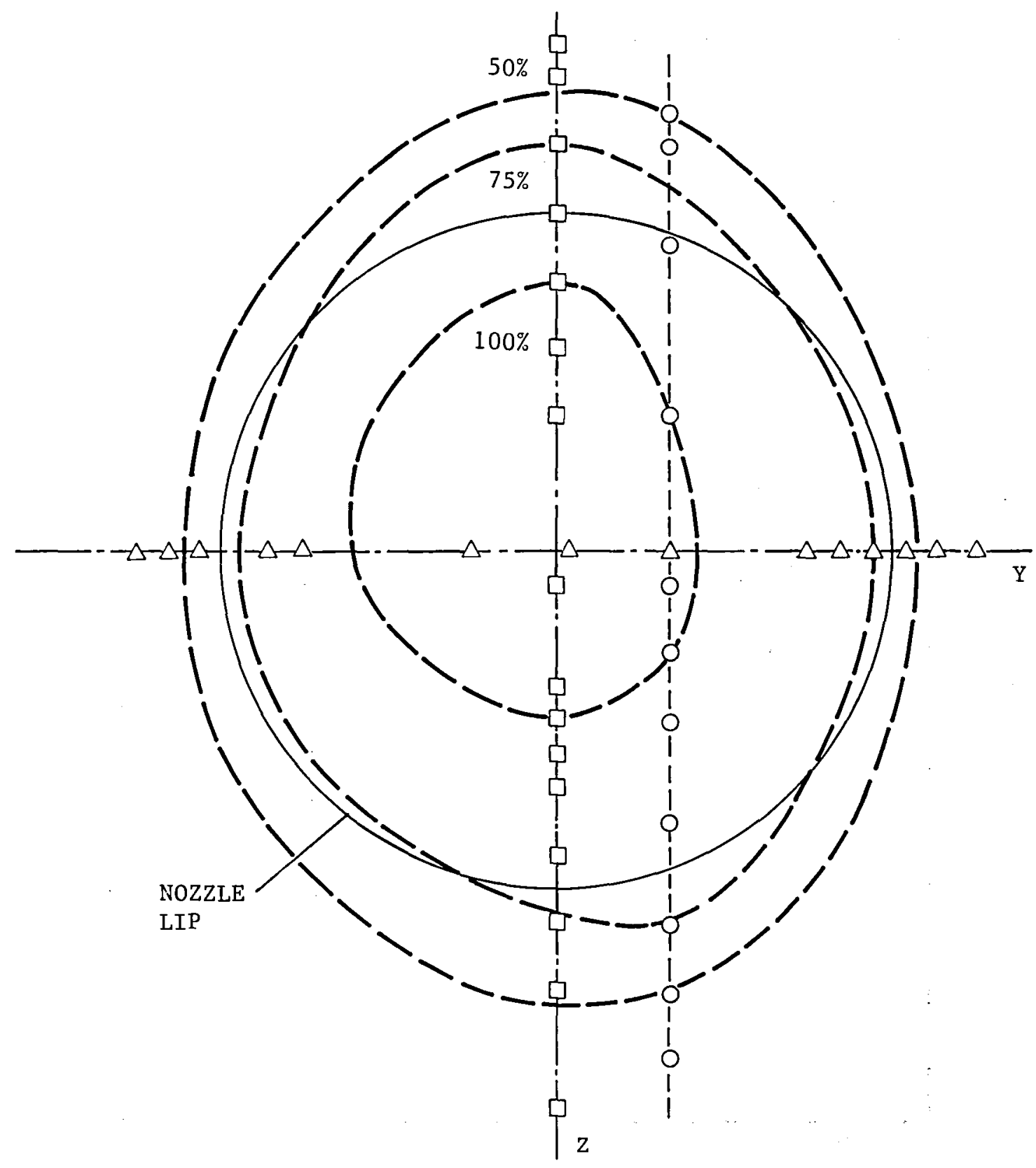

Figure 4.13 Mean velocity contours of a fully expanded, supersonic jet of $\mathrm{M}_{\mathrm{j}}=1.37$ at $\mathrm{X} / \mathrm{D}=3$. 


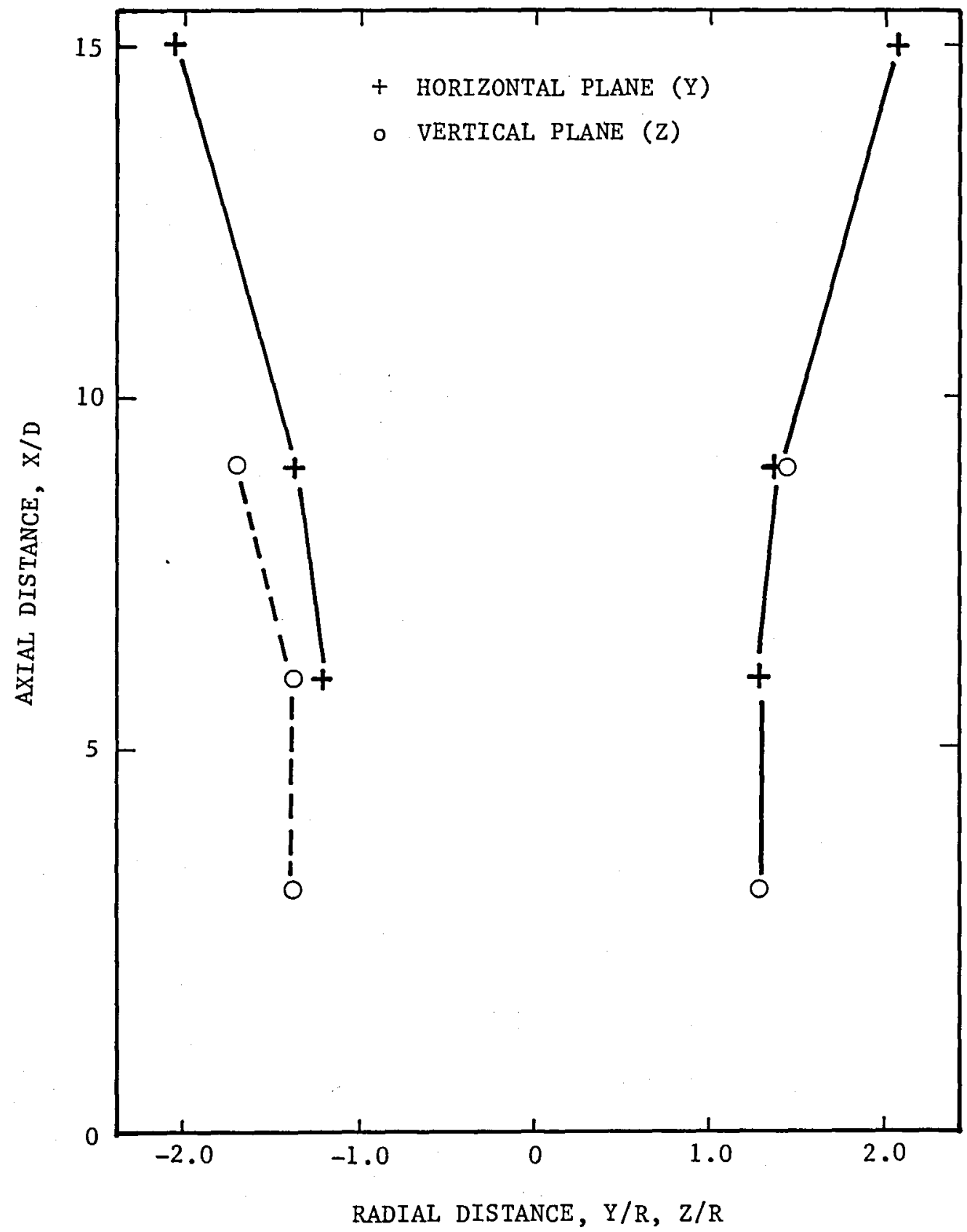

Figure 4.14 A map of half-velocity points in the horizontal and vertical planes of a fully expanded, supersonic jet of $\mathrm{M}_{j}=1.38$ 
for the unexcited and excited, fully-expanded supersonic jets of $M_{j}=1.38$ are given in Figure 4.15. These distributions show again a negligible effect of excitation on the mean flow characteristics. It might be added that this was the precise requirement of this study. The large-scale structure needed to be exited just enough so that it could be visualized, but not strong enough to turn the program into a study of "flow modification by sound."

\subsection{Conditionally Sampled LV Measurements}

Conditionally sampled LV measurements, to generate ensemble-averaged fluctuating velocity distributions, were made at an excitation Strouhal number of 0.4. The levels of excitation used in these tests were equal to those used during the flow visualization task, described in Section 3.0. These levels of excitation were those at which the large-scale structure had become just visible in the flow visualization tests.

The preliminary LV data analysis for these conditions showed, however, that flow had no measurable periodic velocity component. In view of this, it was decided to repeat the experiments, but this time the maximum achievable level of excitation was used. Even for these excitation levels, the mean characteristics of the flow were not modified. These measurements were made along the jet centerline at six axial locations of $X / D=3,5,7,9,11$, and 13. For this excitation level, the periodic velocity component was detected only at the end of the potential core, at $X / D=7$ and 9 ; whereas, at other axial location, no periodic fluctuations were reliably traceable.

As seen in Figure 4.16, there are no periodic fluctuations traceable in the flow at $X / D=5$. At two other locations, $X / D=7$ and $X / D=9$, it is seen that the large-scale structure velocity fluctuates with a period of $T=280$ $s$, which corresponds to the excitation strouhal number of 0.4 . The amplitude of the periodic velocity fluctuations, however, is very small, less than $1 \%$ of jet exit velocity. The velocity amplitude is $4 \mathrm{~m} / \mathrm{s}$ at $X / D=7$ and $3 \mathrm{~m} / \mathrm{s}$ at $X / D=9$. For the jet exit velocity of $454 \mathrm{~m} / \mathrm{s}$, the corresponding intensities of periodic fluctuations are $0.6 \%$ at $X / D=7$ and $0.5 \%$ at $X / D=9$. As seen in Figure 4.15 , the total turbulence intensities are $1.7 \%$, $2.9 \%$, and $5.9 \%$ at the corresponding locations $X / D=5,7$, and 9 . Thus, it appears that the level of periodic fluctuations reaches approximately $20 \%$ of the total turbulence intensity level at $X / D=7$ but only $8 \%$ at $X / D=9$.

Although originally it was planned to acquire considerable data in shear layer, the conditionally-sampled measurements made along the half velocity line in the jet shear layer failed to show a high enough intensity of the large-scale structure. Typical result obtained in this region is shown in Figure 4.17. Here, and as in most measurements in this region, the periodic velocity time distribution is contaminated with very intense background noise attributable to a very low data rate in the supersonic jet shear layer region. Because of the extremely low data rate, almost no data were available in some of the time slots of the resulting file generated during the data reduction procedure of conditionally sampled data (see Figure 2.10 in Section 2.0). Thus, the velocity mean values within some of the time slots are statistically unreliable and exhibit large deviations from correct values and, therefore, 


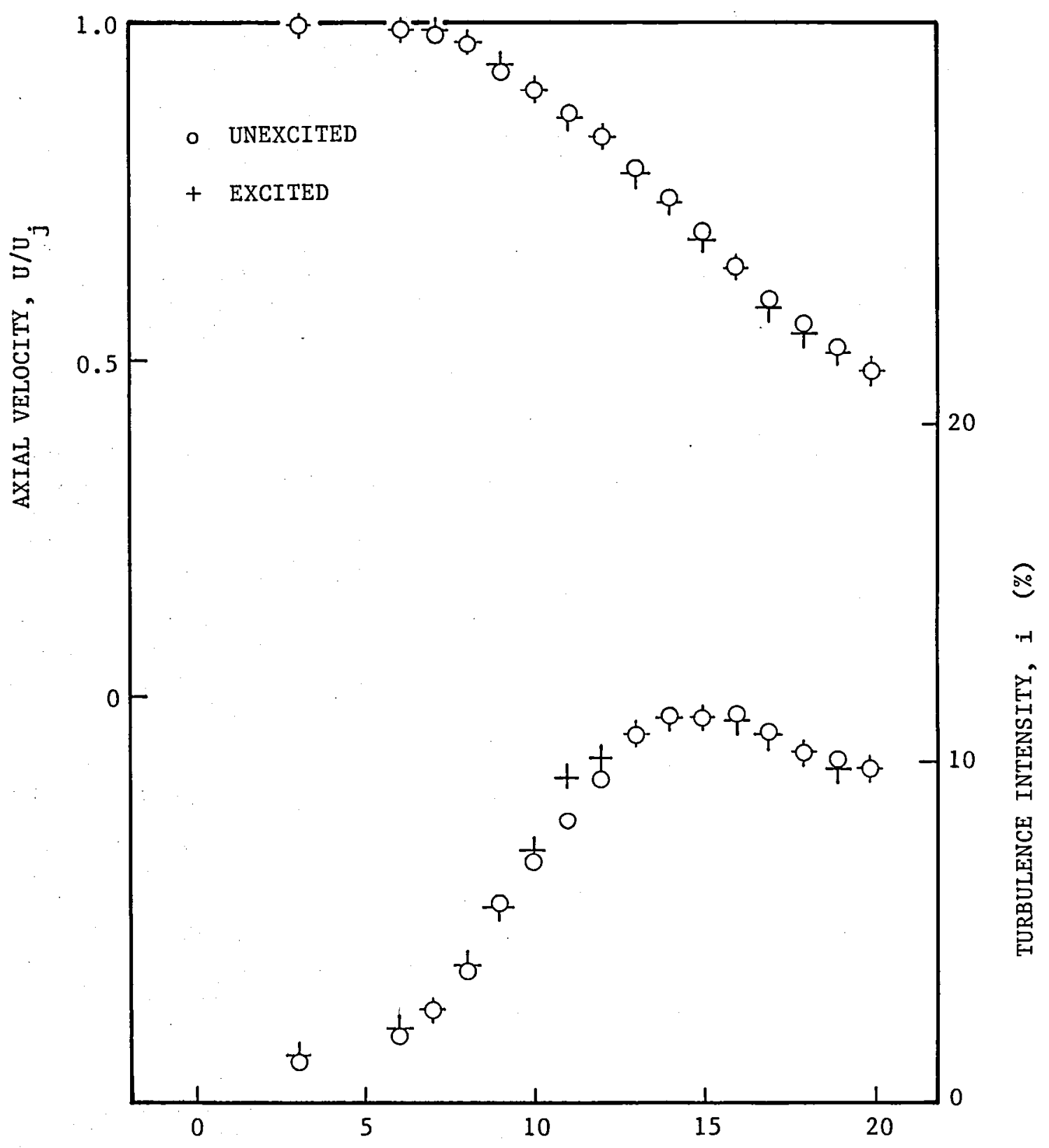

DISTANCE X/D

Figure 4.15. Mean velocity and turbulence itensity centerline profiles of a fully expanded supersonic jet of $\mathrm{M}_{\mathrm{j}}=1.38, \mathrm{U}_{\mathrm{j}}=454 \mathrm{~m} / \mathrm{s}$ 

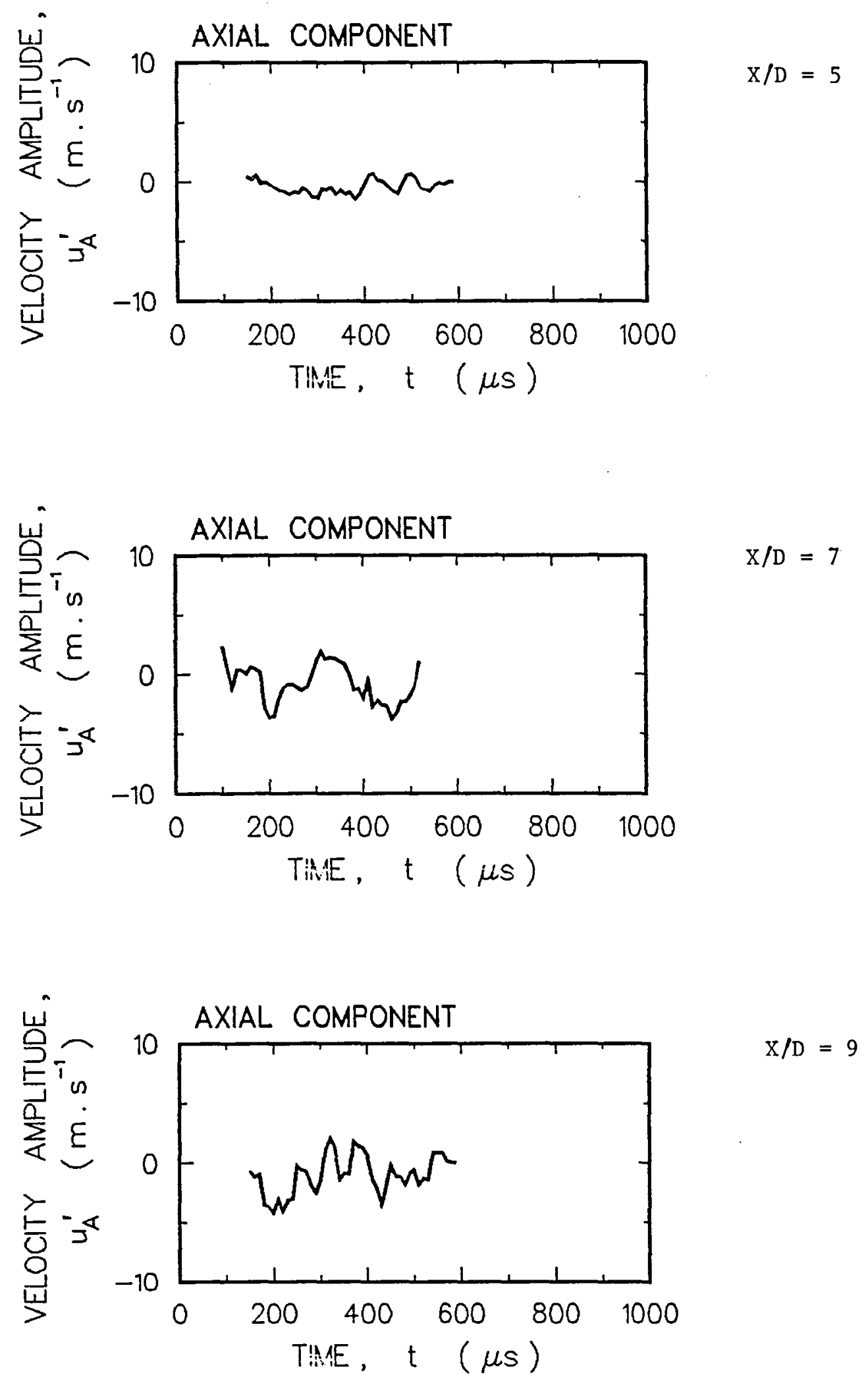

Figure 4.16 Noise contaminated ensemble-averaged velocity data from jet shear layer. 


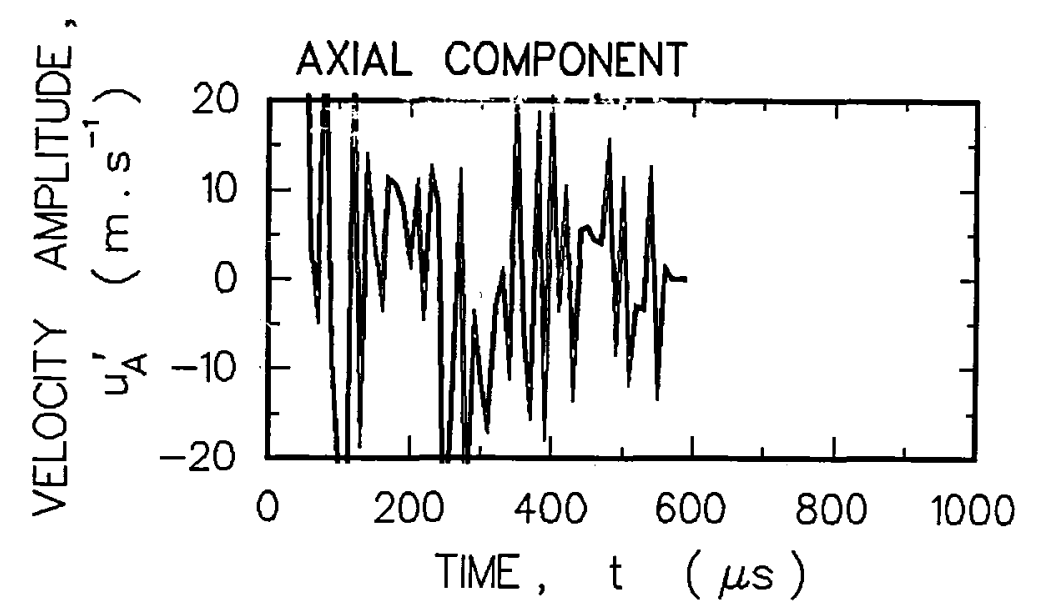

Figure 4.17 Noise contaminated ensemble-averaged velocity data from jet shear layer. 
appear to significantly increase the background noise level. Figure 4.17 is included here just to illustrate the severity of this problem which is related to the extremely low data rate in the jet shear layer, primarily due to an inadequate number of seeding particles in this region. (These problems are further discussed in Section 5.0.)

Based on the results acquired along the jet centerline, however, it appears that the large-scale structures in supersonic, high Reynolds number jets are very weak. This is true, at least, for our given jet operating conditions and for the achievable levels of excitation.

\subsection{Comparison with other Existing Measurements}

Whereas the importance of the large-scale turbulence in subsonic-jet mixing layers is generally recognized and well documented, no experimental results on the behavior of large-scale structures in supersonic, fullyexpanded, high Reynolds number jets have been published in the open literature. Thus, the direct comparison of present experimental data with the results of others is not possible and, therefore, the present data are compared either with the experimental results for low Reynolds number, supersonic jets or with the results for high Mach number, subsonic jets.

\subsubsection{Mean Flow Characteristics}

Two mean flow parameters, namely jet spreading rate and turbulence intensity centerline distribution, were compared with measurements of Morrison and McLaugh1in (Ref. 4.1) and measurements of Lau, et al (Ref. 4.2).

Figure 4.18 shows how the shear layer half thickness parameter $b$ varies with downstream distance for the high Reynolds number jet (present data) and for the low Reynolds number jet (Morrison and McLaughlin, Ref. 4.1). The shear layer half widths at $X / D=6$ and $X / D=9$ are average values computed from horizontal and vertical mean velocity profiles. The width at $X / D=3$ is computed from the vertical velocity profile only, whereas the width at $X / D=$ 15 is computed only from the horizontal velocity profile. Arrows indicate the direction of the expected shift of the width values at these two locations if both, vertical and horizontal, velocity profiles were used for this calculation. As already shown in Figure 4.13, the elliptical velocity contours result in slightly different shear layer width along various radial directions.

When compared with the low Reynolds number jet, the spreading rate of the high Reynolds number jet shear layer is much higher from the beginning and then slows down at the end of the jet potential core. It appears that spreading rates of both, low and high, Reynolds number jets are approximately the same at farther distances from the nozzle exit plane. It appears that the above noted difference between the low and the high Reynolds number jets is real. In fact, Morrison and McLaughlin (Ref. 4.1) reported a similar behavior on comparing their data for low Reynolds number jet of $M_{j}=2.5$, and high Reynolds number jet analytical prediction of Morris and Tam (Ref. 4.3). 
---0--- MORRISON \& MCLAUGHLIN DATA (REF. 4.1)

HIGH REYNOLDS NUMBER JET:

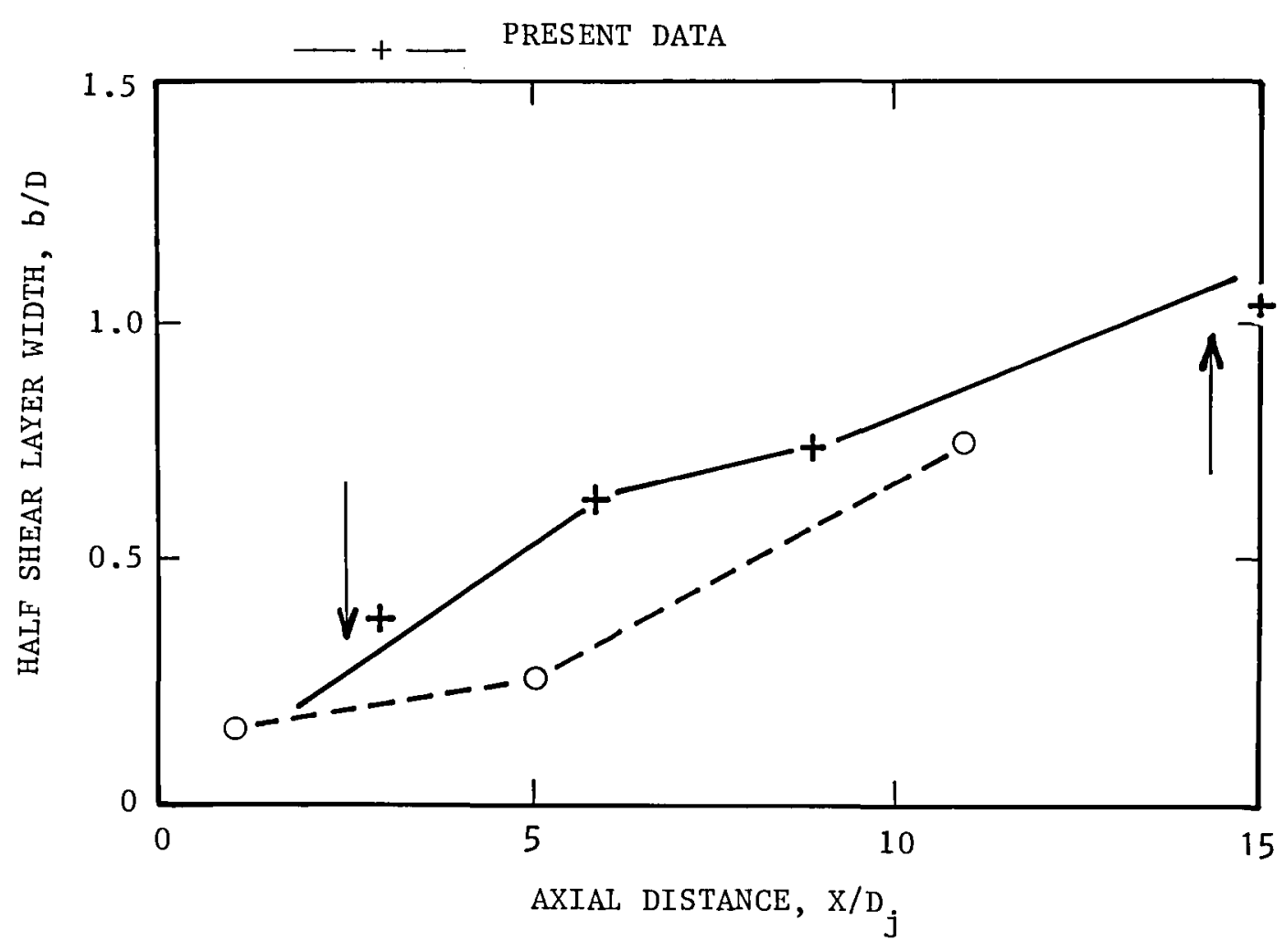

Figure 4.18 Variation of shear layer half-width parameter with downstream distance of a supersonic jet of $\mathrm{M}_{j}=1.4$. 


\subsubsection{Turbulence Intensity}

The centerline distribution of turbulence intensity was compared with similar measurement in high Reynolds number jet of $\mathrm{M}_{j}=1.4$, reported by Lau, et al, (Ref. 4.2). This comparison is shown in Figure 4.19. As seen in this figure, the data agree reasonably well in the region near the end of the jet potential core $(X / D=7)$. Beyond the potential core, in the region of $X / D=$ 10 through 14, the present data tend to show lower turbulence levels than Lau's data. The reason for this discrepancy is not clear.

\subsubsection{Instability Strouhal Number}

In unforced jets, the instability strouhal number is considered to be that which corresponds to the most dominant frequency naturally present in the velocity spectrum. It is believed, that if a jet is excited at this strouhal number, the jet response will be most pronounced. Conversely, the most preferred excitation Strouhal number of a jet should be equal to the jet instability Strouhal number.

Jet instability Strouha1 numbers for low Reynolds number, supersonic jets, measured by Morrison and McLaughlin (Ref. 4.1) are shown in Figure 4.20. The data for high speed subsonic jet, measured by Stromberg, et al (Ref. 4.4) and Armstrong (Ref. 4.5) are al so shown in this figure. It appears that the Strouhal number of the dominant instability has a strong Mach number dependence, particularly in the supersonic region.

Acoustically forced jets, at least for $M_{j}<1.2$, tend to follow a similar trend. The data for acoustically forced subsonic jets, measured by Lepicovsky, et al (Ref. 4.6) are also included in this figure. The data from the present work are also shown in this figure. Clearly, the instability Strouhal number for the present data agrees very well with the results of similar measurements for a range of Reynolds numbers, indicating a similarity of behavior of low and high Reynolds number jets.

\subsubsection{Phase Velocity}

Phase velocity of the dominant instability component as a function of jet Mach number is shown in Figure 4.21. Data in this figure are those measured by Morrison and McLaughlin (Ref. 4.1) in unexcited, supersonic, low Reynolds number jets and by Lepicovsky, et al (Ref. 4.7) measured in a tone excited, subsonic jet of $M_{j}=0.3$. Present data tend to agree very well with the indicated trend.

If, however, the phase velocity is plotted as a function of Strouhal number, a strong disagreement between the forced and the unforced jets is found among the existing experimental data (Ref. 4.5). Figure 4.22 summarizes these findings. It should be mentioned that unlike in Figure 4.21 where the phase velocity was normalized by ambient sound speed, the phase velocity in this figure is normalized by jet exit velocity. In Figure 4.22, the phase velocity variation of unforced jets is shown by a broken line. This line represents the measurements of Armstrong and Ackermann as reported in 


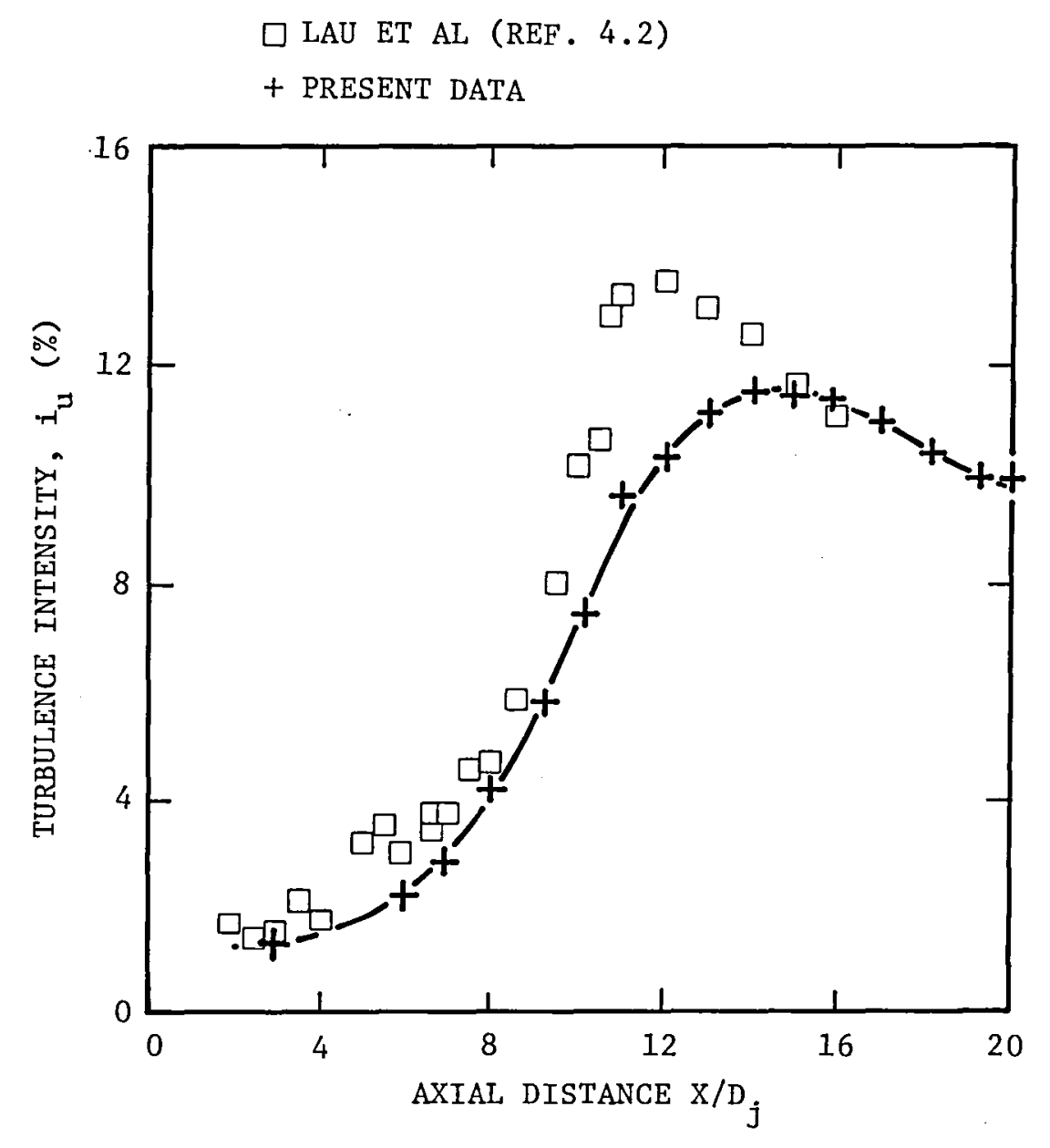

Figure 4.19 Comparison of centerline distributions of total turbulence intensity of supersonic jet of $\mathrm{M}_{j}=1.37$. 
LOW REYNOLDS NUMBER UNFORCED JETS:

O MORRISON \& MCLAUGHLIN (REF. 4.1)

$\triangle$ STROMBERG ET AL (REF. 4.3)

EXC-ITED JETS:

- LEPICOVSKy ET AL (REF. 4.5)

HIGH REYNOLDS NUMBER SUPERSONIC JET:

+ PRESENT DATA

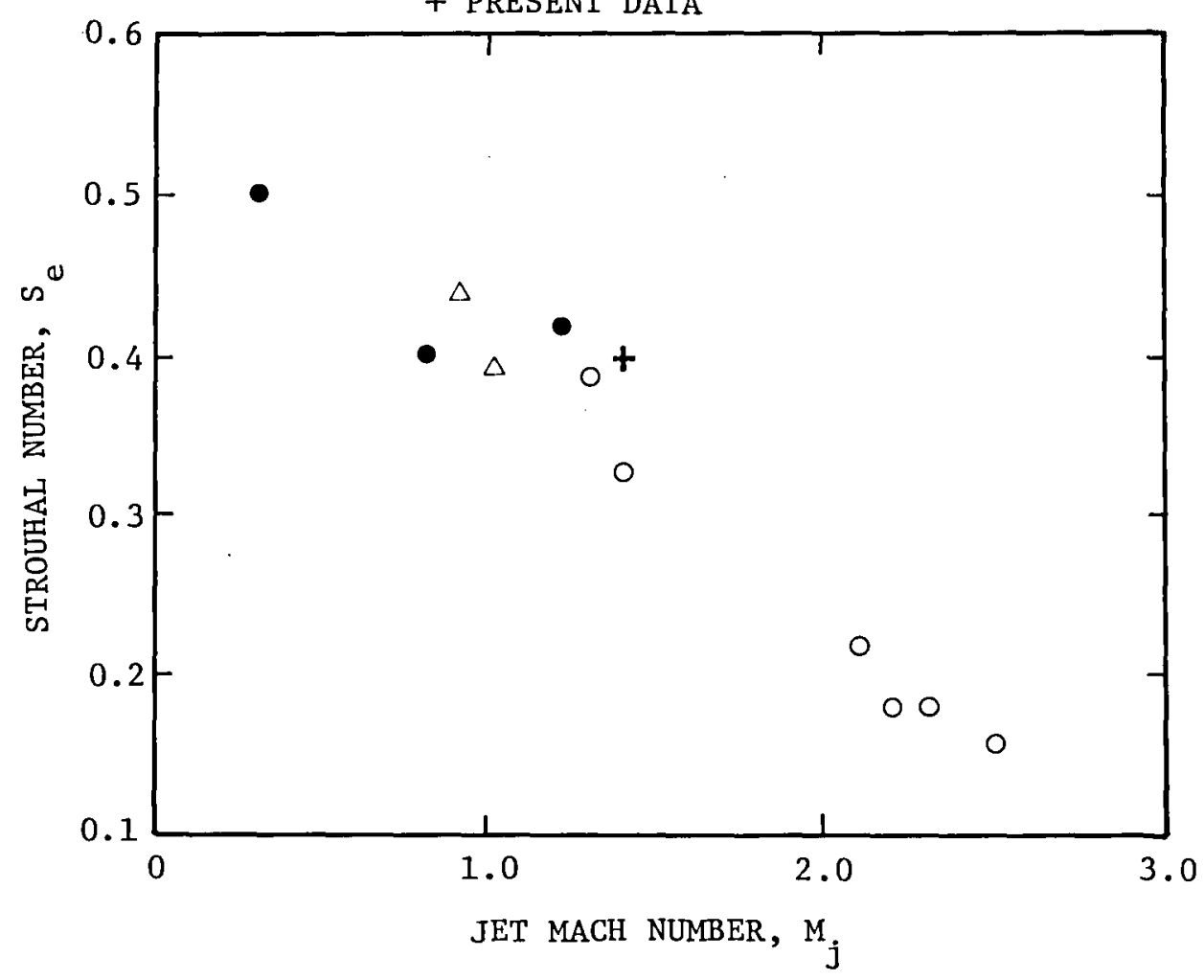


LOW REYNOLDS NUMBER SUPERSONIC JETS:

O MORRISON \& MCLAUGHLIN (REF. 4.1)

EXCITED SUBSONIC JETS:

- LEPICOVSKY ET AL (REF. 4.6)

HIGH REYNOLDS NUMBER SUPERSONIC JET:

+ PRESENT DATA

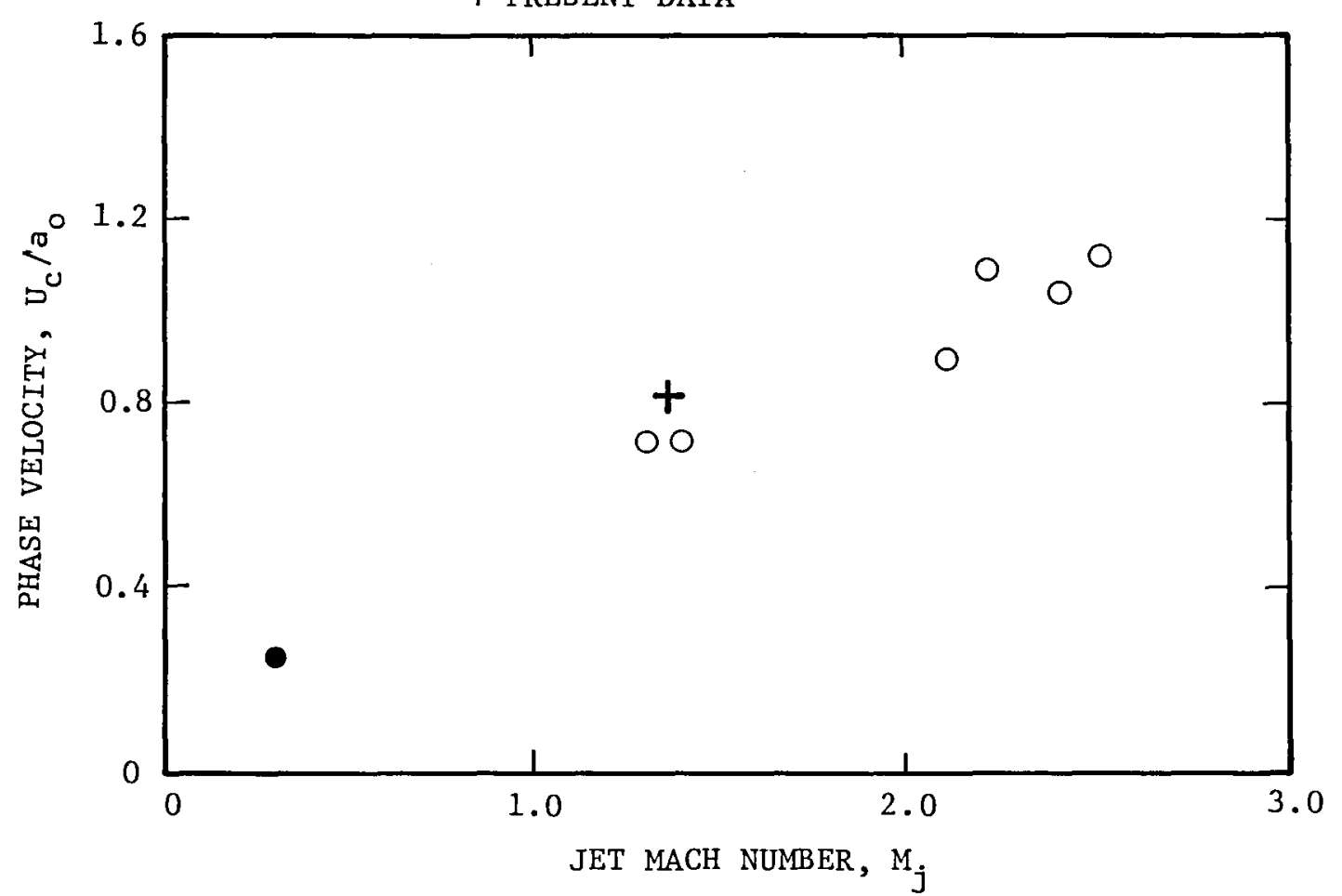

Figure 4.21 Phase velocity of the dominant instability component as a function of jet Mach number. 
UNFORCED JET:

- - - - ARMSTRONG, ACKERMANN (REF. 4.4)

EXCITED JET:

Y//I/I///, CHAN, CROW \& CHAMPAGNE (REF. 4.4)

PRESENT DATA:

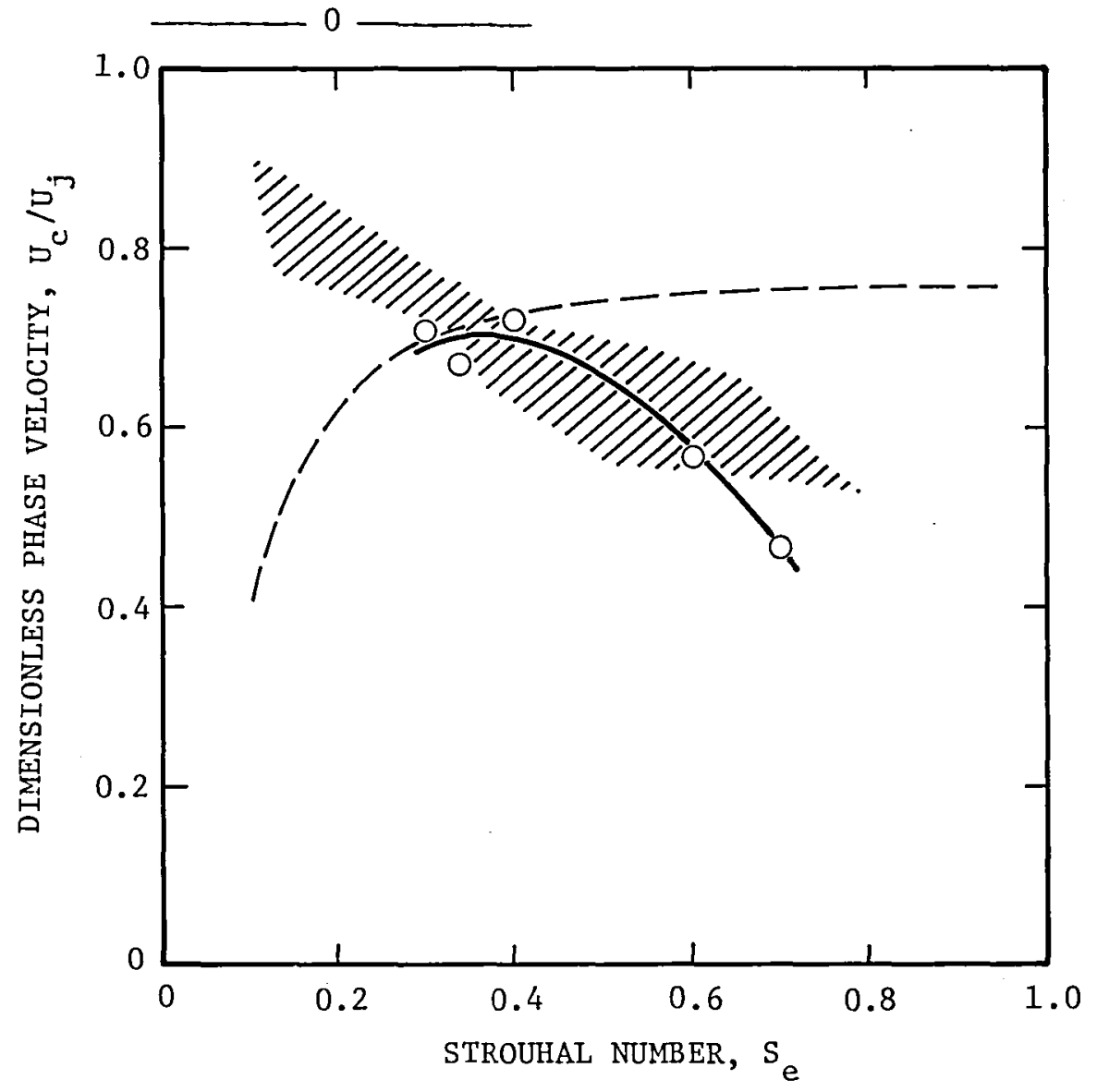

Figure 4.22 Variation of large-scale structure phase velocity with Strouhal number. 
Reference 4.5. Independent measurements in unforced jets in the range of Mach numbers from 0.17 to 0.7 agree very well with each other and show increasing phase velocity with increasing Strouhal number. Measurements in forced jets, however, show decreasing phase velocity with increasing Strouhal number. These measurements, performed by Chan, and Crow and Champagne in low Mach number jets (reported in Reference 4.5), exhibit a higher scatter and are represented in Figure 4.22 by the shaded area. Armstrong (Ref. 4.5) attributes this discrepancy to a basic difference between the behavior of pressure waves in an unforced jet and in an acoustically-forced jet.

As also seen in Figure 4.22, present data for high Reynolds number, supersonic jet of $\mathrm{M}_{\mathrm{j}}=1.37$, exhibit a decrease in phase velocity with increasing Strouhal number.

\subsection{Conclusions}

The purpose of the LV measurement task was to quantify the qualitative results achieved under the flow visualization study described in the previous section. As already mentioned above, the LV measurements have not been completed in the full extent as intended at the beginning of this program. The background of these problems and how we circumvented most of them are given in the next section. However, the results achieved under the LV measurement task, in spite of their lack of completeness, tend to support the findings from the flow visualization task.

Based on the experimental results reported above, the following conclusions may be made:

1. The large-scale structures can be excited in fu1ly expanded, supersonic, high Reynolds number jets, and they prevail even beyond the potential core of the jet.

2. The most preferential excitation Strouhal number for the large-scale structure in fully expanded jet of $\mathrm{M}_{j}=1.4$ is in the vicinity of 0.4 .

3. No significant difference, as far as the preferential Strouhal number is concerned, has been observed between the high and low Reynolds number, fully expanded jets of $\mathrm{M}_{\mathrm{j}}=1.4$.

4. Large-scale structure, excited under the present test conditions, was relatively very weak. The mean flow characteristics were not affected at all by this weak structure. It appears that under these conditions, the level of periodic fluctuations reaches at most $20 \%$ of the total turbulence intensity level on the jet centerline at the end of the jet potential core.

5. The dependence of the large-scale structure phase velocity on the excitation Strouhal number, under the present conditions, shows the same trend as found for subsonic, acoustically excited jets.

6. Relatively good visibility of the large-scale structure in ensembleaveraged photographs and poor "visibility" in LV ensemble averaged velocity 
histories raise questions about the sensitivity of each of the used experimental techniques to the same flow phenomenon.

Summarizing the objectives and results of this study, it may be stated that large-scale coherent-like structures exist in high Reynolds number shockfree supersonic jets. However, to determine their characteristics in detail, further work is needed. 


\subsection{PROBLEMS ENCOUNTERED IN THIS PROJECT, THEIR SOLUTIONS, AND LESSONS LEARNED THEREFROM}

Several serious technical problems were encountered during the course of this project. This section describes these problems and provides an overview of the steps taken to overcome them. Recommendations for future experimental work are also included.

\subsection{Problems with Flow Visualization}

The main difficulty faced during the flow visualization task was the vibration experienced by the framework that held all the optical parts of the laser schlieren setup. The optical frame available at the beginning of flow visualization experiments is shown in Figures 5.1 and 5.2. A schematic sketch of this frame is shown in Figure 5.3. The structure was basically made out of $2 \times 2$ inch, hollow, aluminum members. This framework was successfully used for flow visualization at subsonic jet flow velocities. However, at supersonic jet velocities, particularly at the design Mach number of $M_{j}=1.4$ at which all the planned tests were to be conducted, the framework experienced not only entrainment flow-induced vibrations, but also those due to strong acoustic fields. The sound-induced vibration was particularly felt by the optical pel1icle used in the optical setup. The pellicle was subsequently replaced by a sturdier wedge mirror. The frame vibrations and the resulting relative motion between various optical component, made the acquisition of schlieren pictures of good quality next to impossible. To estimate the severity of this problem, accelerometers were placed on different parts of the framework to determine the extent of the vibrations experienced by the framework. After examining the results, it was concluded that significantly sturdier supports were needed to acquire sharp schlieren pictures at supersonic Mach numbers. It was also decided that, in the long run, considerable time and effort will be saved by discarding the existing framework, and, instead, designing a completely new support.

The newly designed optical supports are shown in Figures 5.4 through 5.6 . Unlike the system shown in Figure 5.1 through 5.3, where a11 structural members were interconnected, the new setup was designed to consist of three independent components. The first, a single-column support, holding the converging mirror and a photographic camera, is made from a 12-in diameter steel pipe and stands on its own rails. The second, a double-column support, holding the auxiliary mirrors, is made out of 10-in. diameter steel pipes and also stands on its own rails. Both supports are fitted with suitable gussets where needed to obtain additional stiffness. In addition, they are filled with sand to reduce the vibration problem further. The third part, the base plate, holds the remaining optical components. The base plate is made of a three-inch thick aluminum plate and sits on the original rails on the top of the existing $\mathrm{LV}$ bed used for traversing $\mathrm{LV}$ frames.

This system of three independent supports proved to be successfu1 for the following two reasons. First, dividing the entire framework in three separate components significantly improved the stiffness of the individual supports and 


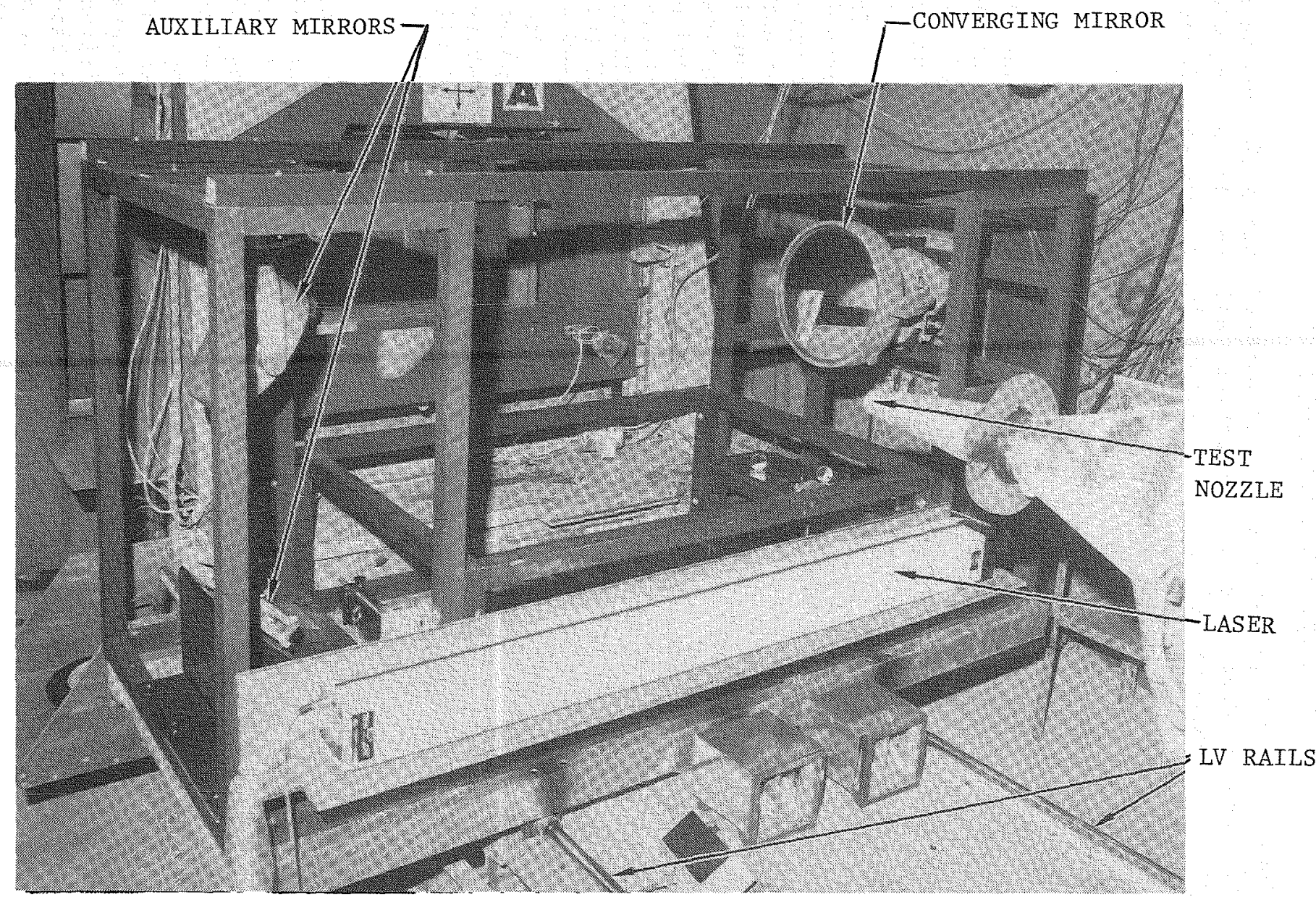

Figure 5.1 old optical framework (left-side view). 


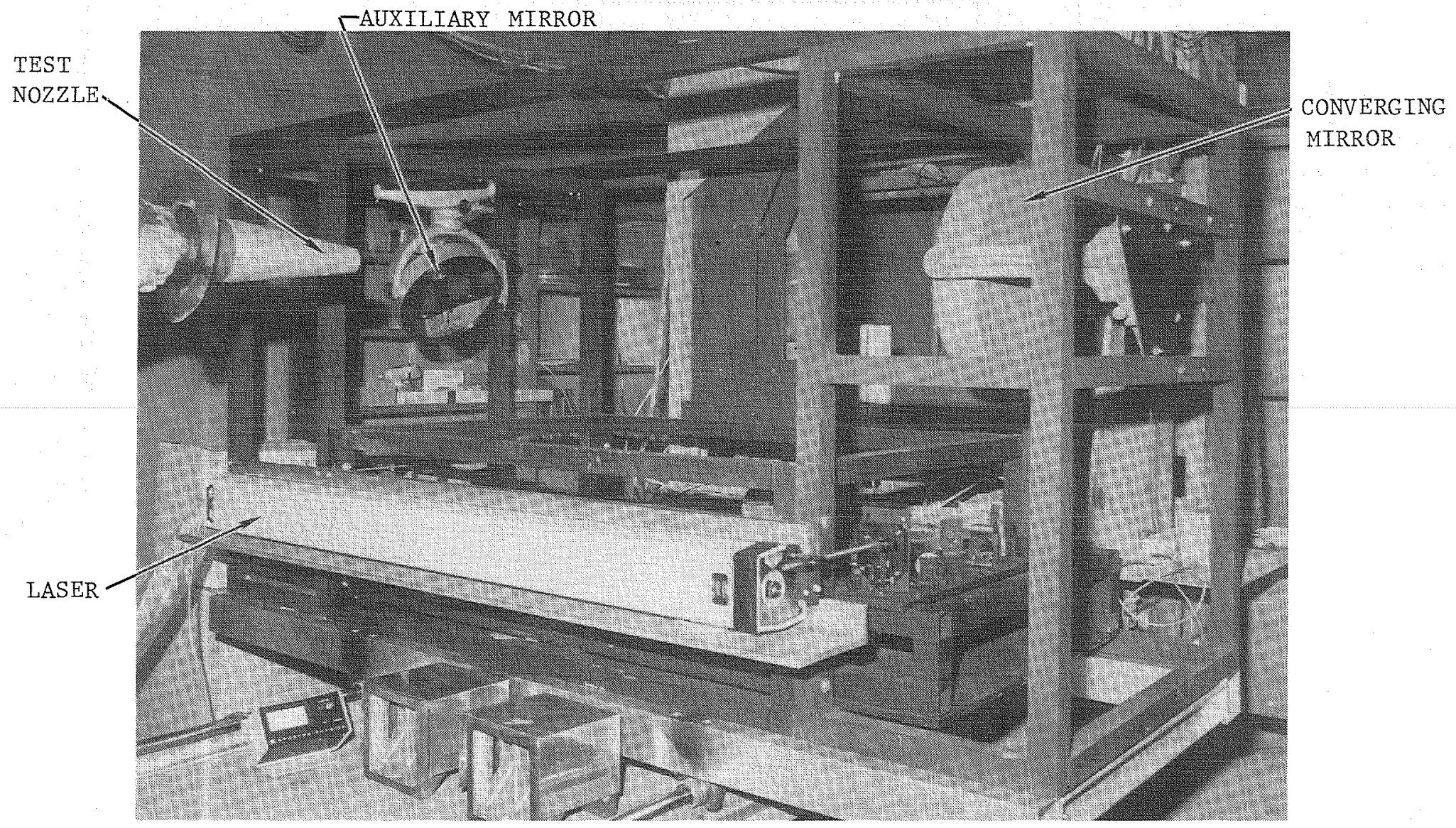

Figure 5.2 old optical framework (right-side view). 


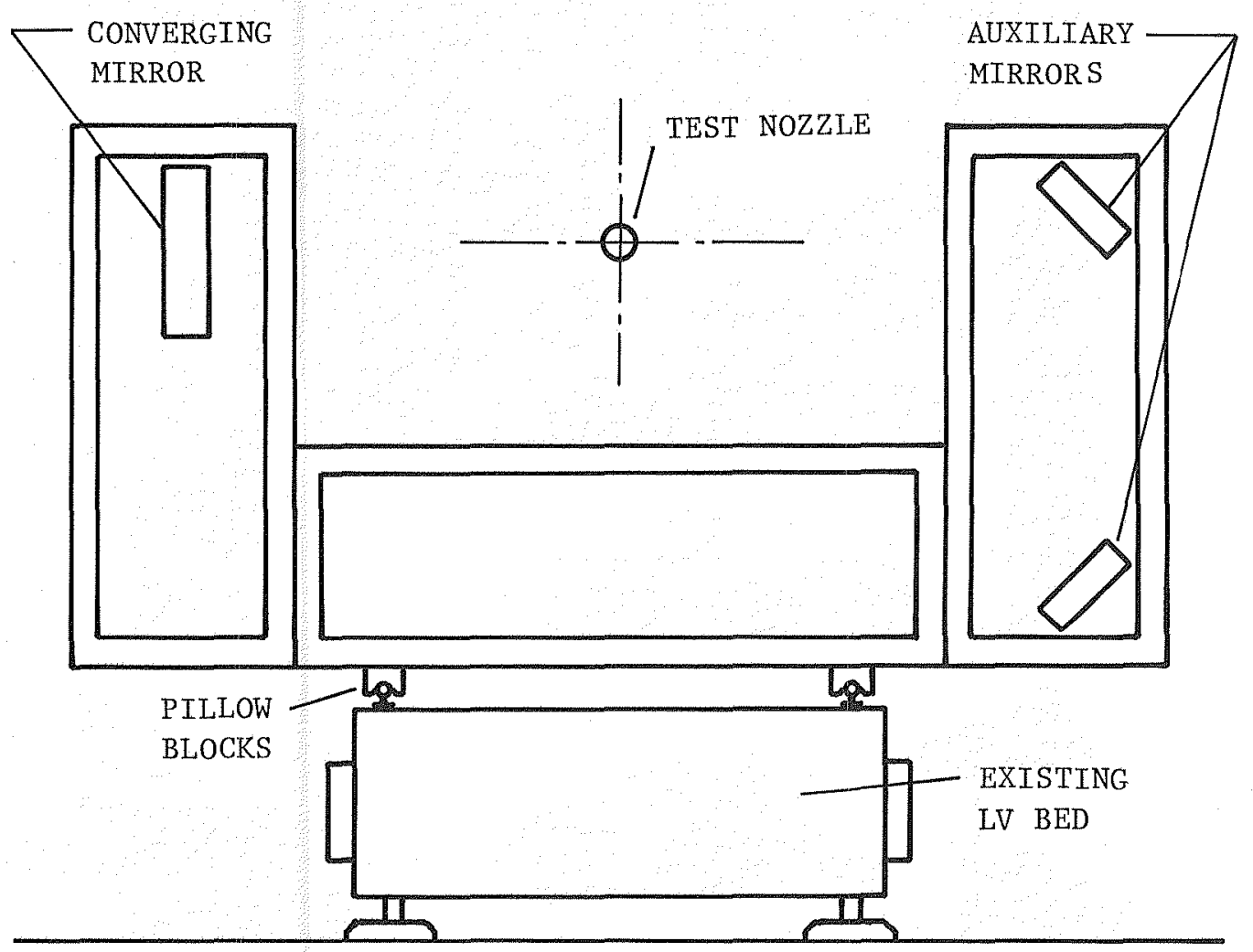

Figure 5.3 Front-view schematic of the old optical framework. 


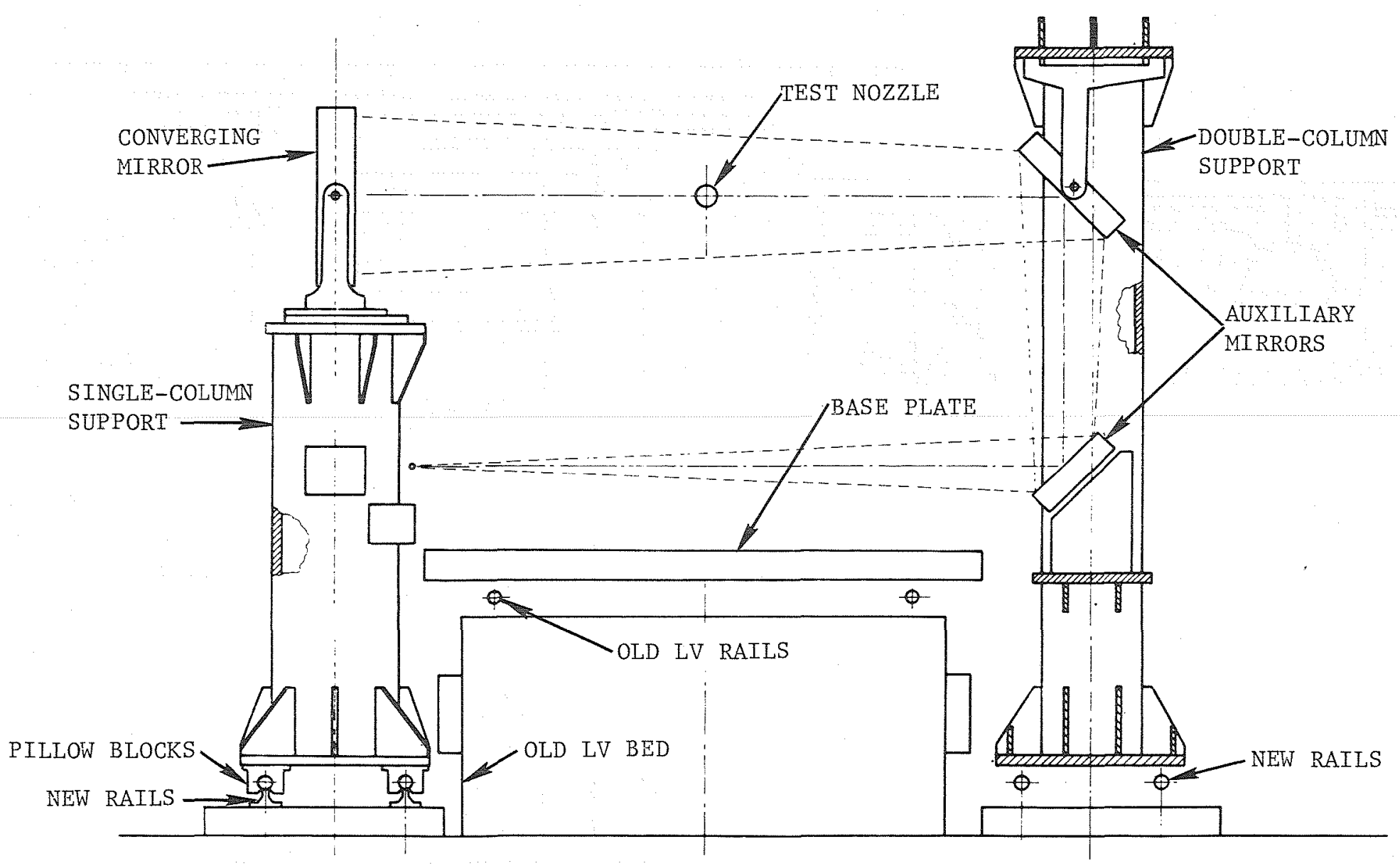




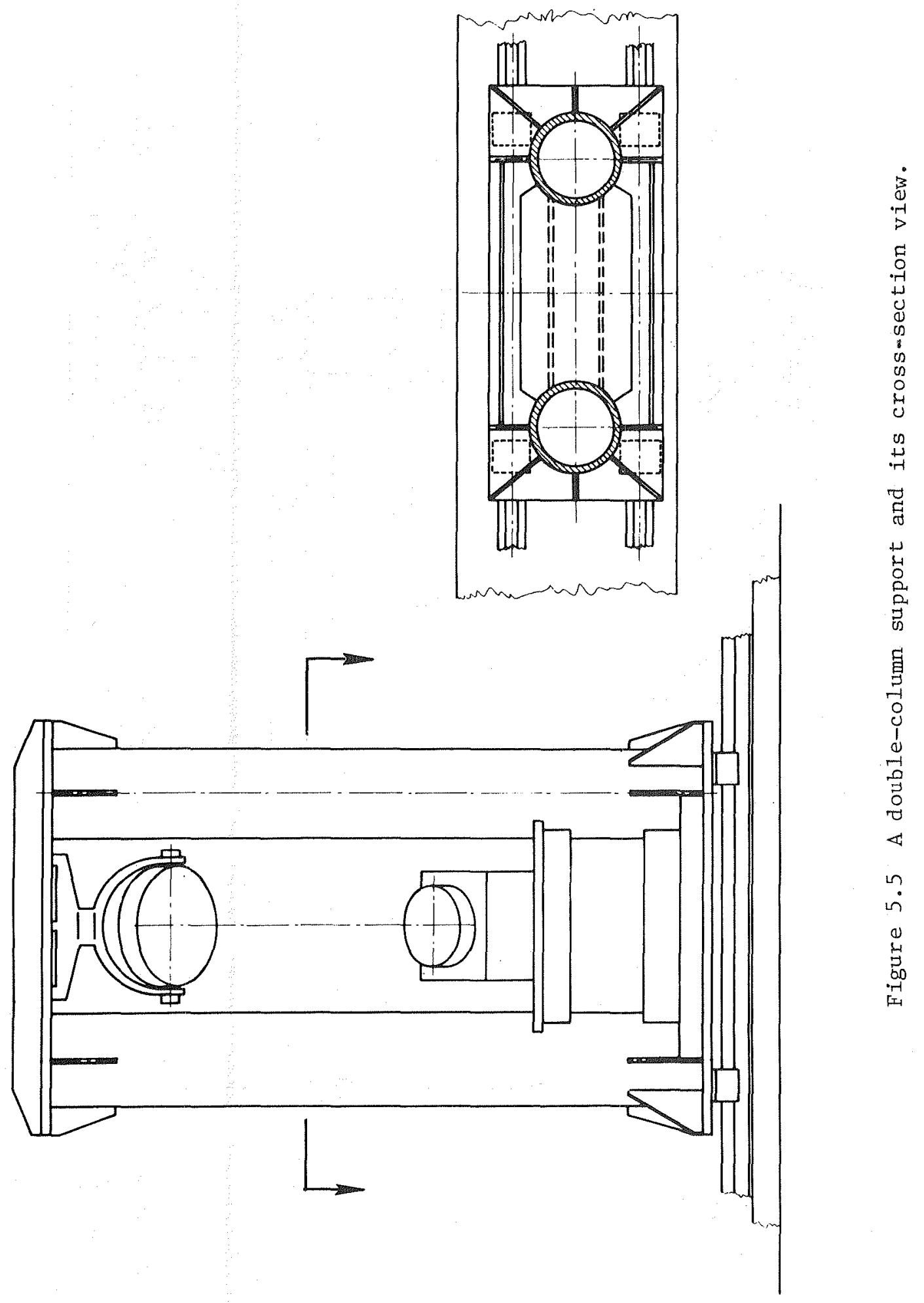




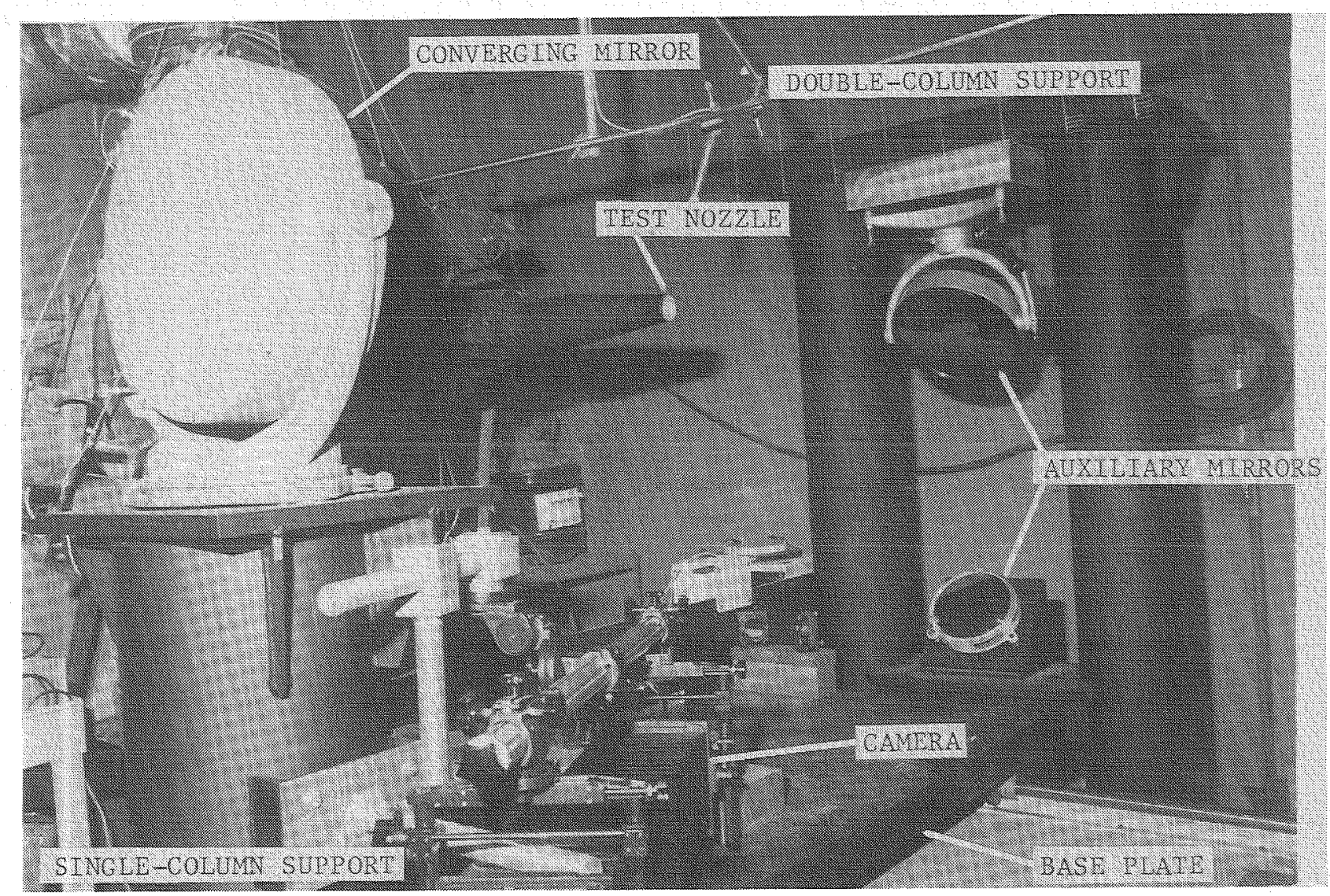

Figure 5.6 Photographic view of the new supports. 
eliminated the vibration problem. Second, the relative location of the independent supports could be changed quite easily now, which made the alignment procedure of the entire optical setup simpler and faster.

\subsection{Problems with LV Measurements}

Quantitative LV measurements in a supersonic jet proved to be more difficult to make than it was originally expected. The problems encountered ranged from a very poor signal to noise ratio of the LV signals at supersonic velocities, to those related to laser tube breakage and electronics in general. The more important problems are summarized below.

\subsubsection{Signa1-to-Noise Ratio Problem}

A poor signa1-to-noise ratio generally leads to low validated LV data rates and to increased measurement errors. A low validated data rate does not prevent the acquisition of reliable flow mean velocity and turbulence intensity data because only the "velocity" data are stored into the processor memory in this data acquisition mode. The mean velocity and turbulence intensity are computed only after a sufficient number of "velocity" points are stored in the data bank.

However, to acquire conditionally sampled data for ensemble averaging, a high validated data rate is definitely desirable. This is because in this mode "velocity" as we11 as "time" data are acquired and stored in the processor memory. There are two types of "time" data. The first one is the time between successive validated "velocity" data and the second one is that associated with the triggering frequency.

Thus, in conditional sampling mode, only a part of the processor memory is available for the "velocity" data because the "time" data also need to be stored in the same memory. It must be borne in mind that time data have to be acquired and stored continuous $1 y$ so as not to lose the track of the velocity history. This is true even when no velocity data are recorded between two successive triggering pulses. Thus, at low validated velocity data rate, most of the processor memory may be occupied by the "time" data, thus rendering the memory capacity for "velocity" data inadequate to accommodate a sufficient number of "velocity" data for reliable velocity measurements. This problem becomes even more critical if the acquired set of data is to be used for autoor cross-correlations and frequency spectra computation.

In general, there is no commonly valid fixed ratio of validated data rate and triggering frequency assuring that the conditionally sampled velocity data are jitter free. This ratio depends mainly on the total available memory capacity. It also depends on the number of velocity components acquired simultaneously. Further, the required time resolution on the conditionally sampled velocity history also plays a very important role. Finally, this ratio is also a function of the overall efficiency of the numeric data transfer and storage. In all the above mentioned cases, however, the high validated data is essential for overall improvement of the accuracy of conditionally sampled velocity measurements. Therefore, our main effort was devoted to the improvement of the validated LV data rate. 


\section{Overcoming the Fogging Problem}

A certain improvement of the validated data rate was achieved by operating the jet at iosothermal conditions to avoid fogging of the jet plume. Excessive fogging of the jet plume prevents laser velocimeter measurements because the intense background light, scattered from the fog, completely overwhelms the light signal from the seeding particles tracing the flow. Total temperature of the flow was kept at $370 \mathrm{~K}$, which is just above the "fogging" temperature of the jet. Nevertheless, the signal-to-noise ratio remained at a relatively low level which enabled us to acquire routinely only data for the axial velocity component in the vicinity of the jet centerline. We were, however, still unable to reach sufficient laser velocimeter data rates for conditional sampling in the jet shear layer and for measuring radial velocity component at high jet velocities.

\section{Increasing the Seeding of the Flow}

Provided that the LV optics are properly aligned and the LV electronics are properly tuned, the low validated LV data rate may be an indication of an insufficient number of tracing particles in the flow. Thus, the next logical step was an attempt to enhance the seeding of the flow. It has to be emphasized here, that our test facility is an open loop one; thus, all the tracing particles seeded into the flow cross the LV measurement volume only once and then they are lost. This is a significant disadvantage in comparison with a closed loop test facility in which one can gradually increase the number of tracing particles, using even a low performance seeder until the desired number of particles in the flow is reached. An open loop test facility requires an extremely powerful seeder, particularly at very high flow velocities.

To seed the flow, a high volume fluidized bed aerosol generator (model TSI 9310) was used. This generator, specifically designed as a tool for use in high volume LV seeding, is the most powerful LV seeder available in the market. Operating the seeder at a very high inlet pressure ( $20 \%$ higher than is the maximum rated inlet pressure) provided additional improvement of the validated $L V$ data rate and enabled us to perform conditionally sampled data acquisition along the jet centerline. Branching the seeder output for a simultaneous internal and external seeding enabled us to acquire mean velocity and turbulence intensity data in the jet shear layer even beyond the half velocity point.

Despite the above mentioned improvements, the validated $\mathrm{LV}$ data rate in the jet shear layer was still insufficient to make reliable conditionally sampled measurements and frequency spectra measurements in this region of the supersonic jet plume. Also, the reliability of the radial velocity and radial turbulence intensity components remained in question.

Therefore, we could not perform all of the LV measurements as originally intended. The LV data presented in this report are only those in which we have full confidence. 


\subsubsection{Hardware-Related Low Data Rate Problem}

It is shown in this subsection that for $L V$ measurement in supersonic jets, particularly for conditional sampled measurements of low amplitude large-scale structure, the velocity resolution of the LV system needs to be improved considerably. It is shown that this can be done by using an ultra high frequency, on the order of $1 \mathrm{GHz}$, clock. To prove this point, the relevant background on the workings of the related LV hardware is given first.

A laser velocimeter measures flow velocity by measuring the frequency of the scattered light signal generated by particles in the flow crossing the fringe pattern in the measurement volume. The flow velocity is computed from this measured light signal frequency and the known distance between fringes in the measurement volume. Frequency of the scattered light signal, monitored by a photomultiplier tube, depends on three factors: flow velocity, fringe spacing, and Bragg cell frequency. In our particular arrangement for a flow velocity of $450 \mathrm{~m} / \mathrm{s}$, the resulting frequency of the scattered light is about $60 \mathrm{MHz}$ (Bragg cell frequency is $45 \mathrm{MHz}$, fringe spacing is 30 micron). The Lockheed LV counter processor measures this frequency using a $500 \mathrm{MHz}$ clock such that the clock measures a time interval corresponding to eight signal periods (or eight fringes in this case). For $60 \mathrm{MHz}$ signal, this time interval is equal to $133.3 \mathrm{~ns}$. The $500 \mathrm{MHz}$ clock has a resolution of $2 \mathrm{ns,}$ which means that the count representing the $60 \mathrm{MHz}$ signal is equal to $66+1$. Thus, the resolution of measured velocity is $\pm 1.5 \%$ and it is $\pm 6.8 \mathrm{~m} / \mathrm{s}$ at a velocity level of $450 \mathrm{~m} / \mathrm{s}$. This resolution is acceptable for mean velocity and mean turbulence intensity measurements, but is absolutely unacceptable for conditionally sampled measurement of small-amplitude periodic-velocity fluctuations because such resolution was too coarse, as further elaborated below.

As shown in the previous section, the periodic velocity component, associated with the large-scale turbulence structure, is relatively weak in our case, and its amplitude is about $4 \mathrm{~m} / \mathrm{s}$. Thus, to be able to measure velocity fluctuations of this order, the velocity resolution must be increased at least to a level of $+2 \mathrm{~m} / \mathrm{s}$. There are two solutions to increase the $\mathrm{LV}$ counter processor resolution. The first solution, relatively common, is to beat down the signal frequency with an independent constant frequency signal. The second solution is to use a clock with higher resolution of say, 1 ns ( 1 $\mathrm{GHz}$ clock). Since the second solution represents a major change in the Lockheed LV hardware and software systems, the improvement of the velocity resolution by down beating the signal frequency was used.

A photomultiplier signal was mixed with a constant $40 \mathrm{MHz}$ signal to produce resulting $20 \mathrm{MHz}$ signal at the input of the $\mathrm{LV}$ counter processor. This results in a velocity count of $200 \pm 1$. The corresponding velocity resolution was $\pm 0.5 \%( \pm 2.2 \mathrm{~m} / \mathrm{s}$ for $450 \mathrm{~m} / \mathrm{s})$.

The above mode of operation increases the time required for signal validation. The time interval for 8 periods of $20 \mathrm{MHz}$ signal now is $400 \mathrm{~ns}$. A particle in the flow has to generate a valid signal much longer than before. In reality, it means that a particle has to cross 24 fringes "without an 
error" instead of the previous 8 fringes. It is clear that the probability that "something may go wrong" during the data validation process has increased. This results in a decreased validated data rate.

There is another unfavorable aspect of the longer validation time interval. The measurement volume generated by the intersecting laser beams has a diameter of about 200 micron. A particle which crosses the measurement volume at its center at a velocity of $450 \mathrm{~m} / \mathrm{s}$ resides in this volume only 440 ns. Because the required validation time for the high velocity of $450 \mathrm{~m} / \mathrm{s}$ is now 400 ns, a particle has to cross at 1 east $90 \%$ of the measurement volume diameter to be properly validated. (The percentage length of the measurement volume, required to be traveled by a particle, is given as the ratio of the time for signal validation versus the particle residing time in the measurement volume.) This is a very severe limitation, and in fact further reduces the validated data rate. Particles crossing the measurement volume farther from its center, or particles for which the validation process started later than that for the first $10 \%$ of the measurement volume will be rejected, even if the signals are perfect, just because the validation procedure cannot be completed.

In summary, although the use of the beat-down-frequency approach to improve the velocity resolution to be able to measure large-scale turbulence velocity as low as $4 \mathrm{~m} / \mathrm{s}$ is needed, it results in a reduction of validated data rate for flow velocities for which the validation time approaches the particle residing time in the measurement volume. In essence, it increases the total measurement time required for conditional sampling, while at the same time degrades the capability of acquiring adequate turbulence spectra.

To improve the velocity resolution at supersonic velocities in future experiments without the attendant negative feedback on validated data rate, an effort has been initiated at Lockheed-Georgia Company to introduce an LV counter processor with $1 \mathrm{GHz}$ clock in our LV system.

\subsubsection{Frequency Response}

The experiments described in this subsection demonstrate the frequency response of the laser velocimeter. Originally, it was intended to demonstrate LV frequency response by measuring velocity spectra in the shear layer of the fully expanded supersonic jet of $M_{j}=1.38$. However, the difficulties with a low validated LV data rate in supersonic jet, as described in the previous subsections, prevented us from this direct measurement. Thus, the LV frequency response is demonstrated in a set of velocity spectra measurements performed in the shear layer of a subsonic jet of $M_{j}=0.9$.

The velocity spectra measurements were made along the lipline of a 50.8mm-dia convergent nozzle at five different axial locations. The mean velocity and turbulence intensity distributions are shown in Figure 5.7. Both distributions show only the axial component of the mean velocity and turbulence intensity. 
The turbulence spectra measured at different axial locations are plotted in Figure 5.8. To verify a collapse of these spectra to a universal shape, the individual spectra have to be properly scaled. The spectra were scaled with respect to the local strouhal numbers and corresponding axial component of the local overall turbulence intensity. The scaled spectra are shown in Figure 5.9. As seen, the collapse is quite good and comparable with the collapse for the hot-wire spectra taken by Seiner and Norum (Ref. 5.1), in terms of spectrum shape, signal-to-noise ratio, and envelope width variation with Strouhal number. This collapse of the free jet spectra indicates that as far as frequency response is concerned, the laser velocimeter fulfills the basic instrumentation requirements to conduct quantitative measurements in high Reynolds number jets, as high as $M_{j}=0.9$, and with further improvement in signal-to-noise ratio, should provide adequate spectra for the supersonic jets also.

\subsection{Concluding Remarks}

This section summarizes the major technical problems during the course of the experimental work and describes the attempts to define and implement proper solutions under the given circumstances. A review of the lessons learned by us should prove beneficial to other users of an LV system for research programs similar to the one described here. 

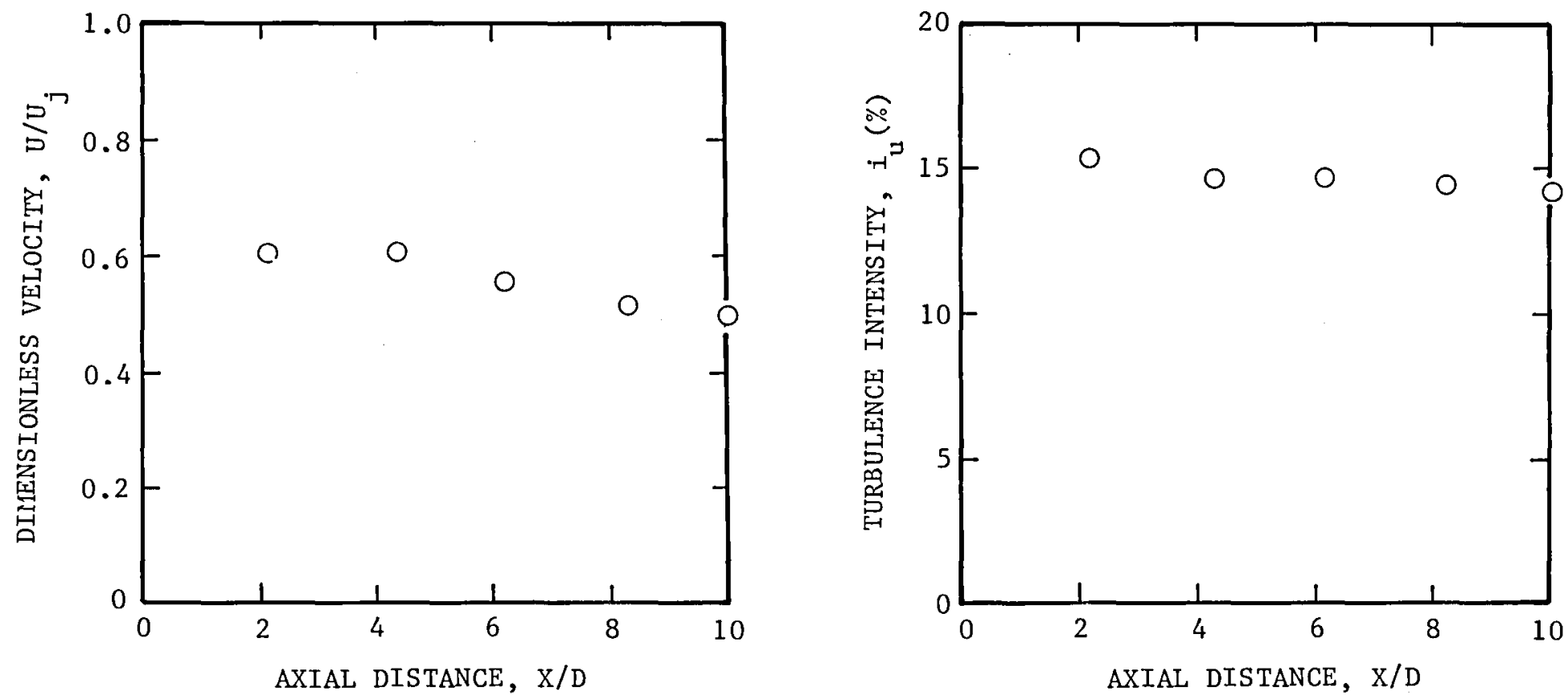

Figure 5.7 Axial mean velocity and axial turbulence intensity 1ipline distributions of a free jet of $\mathrm{M}_{j}=0.91$. 


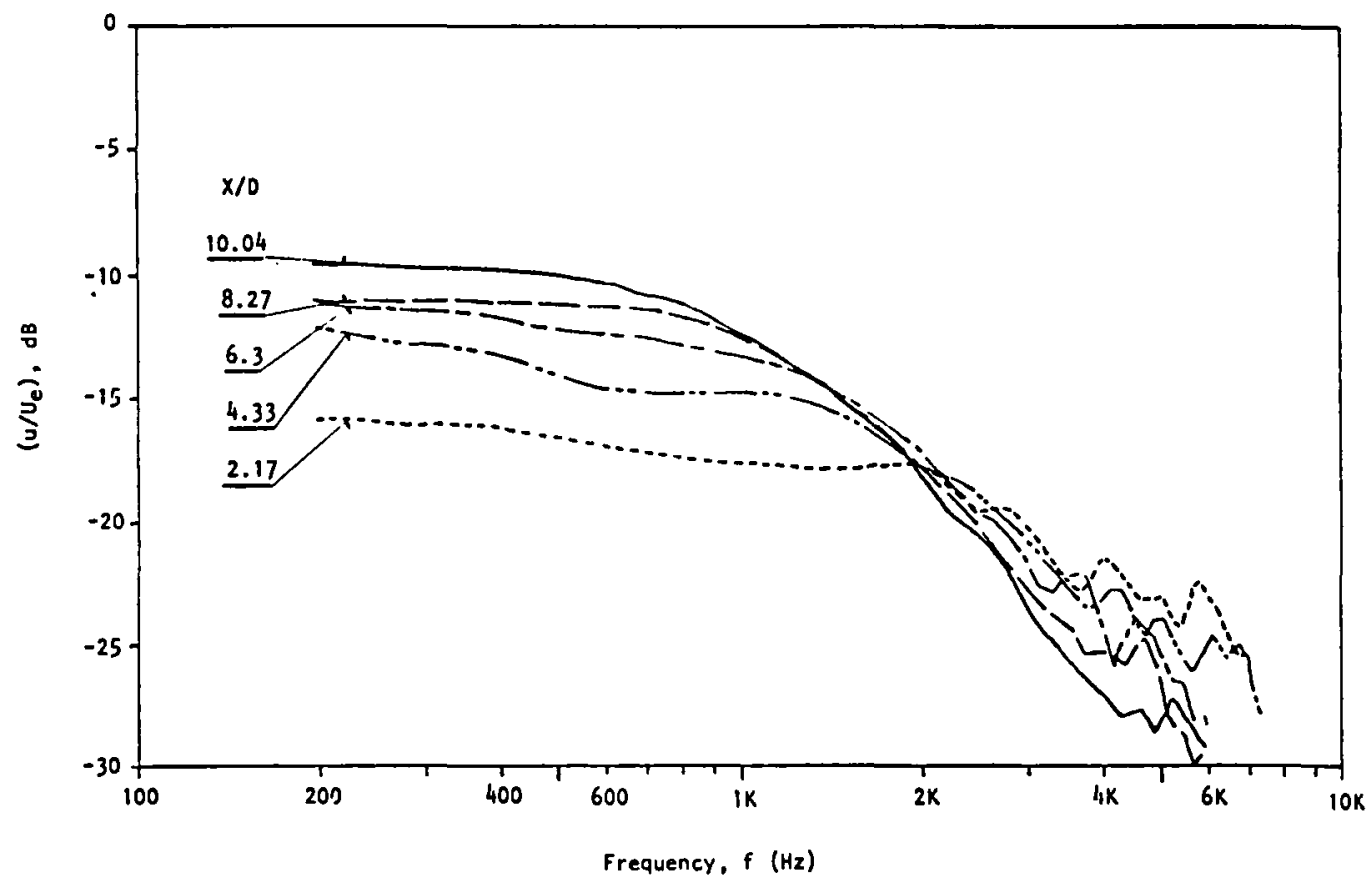

Figure 5.8 Lipline distribution of the turbulence power spectra $\left(M_{j}=0.91\right)$

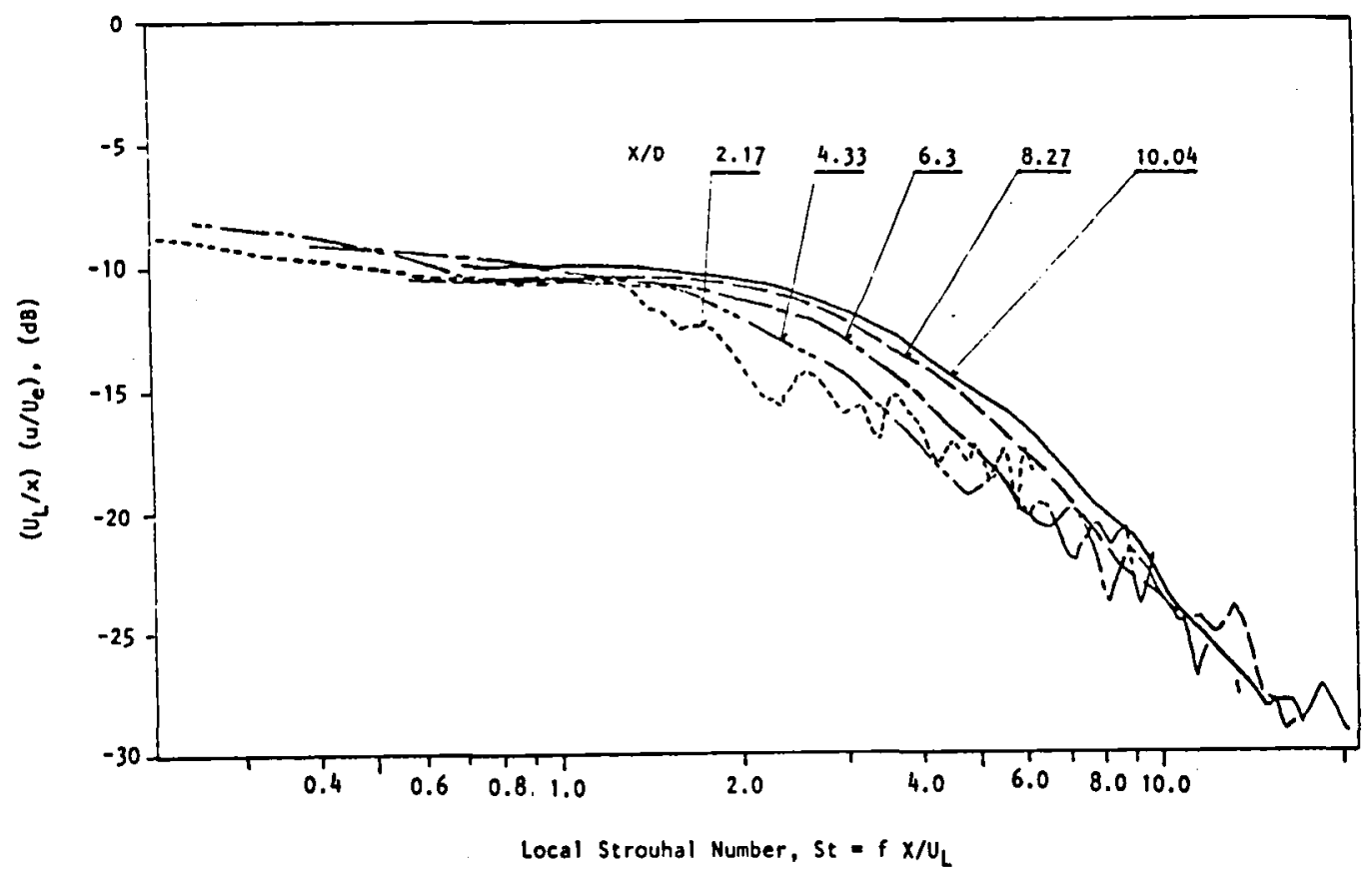

Figure 5.9 Lipline distribution of the turbulence power spectra $\left(M_{j}=0.91\right)$ 


\section{REFERENCES}

1.1 Townsend, A. A.: The Structures of Turbulent Shear Flow, Sambridge University Press, 1956.

1.2 Crow, S. C.; and Champagne, F. H.: Orderly Structure in Jet Turbulence, J. Fluid Mech., Vo1. 48, 1971, pp. 547-591.

1.3 Brown, G. L. ; and Roshko, A.: The effect of density differences on the turbulent mixing layer. Turbulent Shear Flows, AGARD Current Paper No. 93, 1971 pp. 1-23.

1.4 Brown, G. L.; and Roshko, A.: On density effects and large structure in turbulent mixing layers. J. Fluid Mech.,Vo1. 64, 1974, pp. 775-816.

1.5 Roshko, A.: Structure of turbulent shear flows: a new look. AIAA Journa1, Vo1. 14, 1976, 1349-1357.

1.6 Cantwell, B. J.: Organized motion in turbulent flow. Annual Review Fluid Mech., Vo1. 13, 1981, pp. 457-515.

1.7 Hussain, A. K. M. F.: Coherent structures - reality and myth. Phys. Fluids, Vol. 26, 1983, pp. 2816-2850.

1.8 Ho, C. M.; and Huerre, P.: Perturbed Free Shear Layers. Ann. Rev. Fluid Mech., Vol. 16, 1984, pp. 365-424.

1.9 Sede1'nikow, T. K.: The frequency spectrum of the noise of a supersonic jet. Phys. Aero. Noise Nauka (Trans1. 1969 NASA TTF-538, pp. 71-35), 1967.

1.10 Tam, C. K. W.: Directional acoustic radiation from a supersonic jet generated by shear layer instability. J. Fluid Mech., Vol. 46, 1971, pp. 757-768.

1.11 Tam, C. K. W.: On the noise of a nearly ideally expanded supersonic jet. J. F1uid Mech., Vo1. 51, 1972, pp. 69-95.

1.12 Tam, C. K. W.: Supersonic jet noise generated by large-scale disturbances. J. Sound Vib., Vol. 38, 1975, pp. 51-79.

1.13 Bishop, K. A.; Ffowcs Williams, J. E.; and Smith, W.: On the noise sources of the unsuppressed high speed jet. J. F1uid Mech., Vol. 50, 1971, pp. 21-31.

1.14 Morris, P.J.: Flow characteristics of the large-scale wave-like structure of a supersonic round jet. J. Sound Vib., Vo1. 53, 1977, pp. 223-244. 
1.15 McLaugh1in, D. K.; Morrison, G. L.; and Troutt, T. R.: Experiments on the instability waves in a supersonic jet and their acoustic radiation. J. Fluid Mech., Vol. 69, 1975, pp. 73-95.

1.16 Mcluaugh1in, D. K.; Morrison, G. L.; and Troutt, T. R.: Reynolds number dependence in supersonic jet noise. AIAA Journal, Vo1. 15, 1977, pp. 526-532.

1.17 Troutt, T. R.; and McLaughlin, D. K.: Experiments on the flow and acoustic properties of a moderate-Reynolds-number supersonic jet. $\mathrm{J}$. F1uid Mech., Vol. 116, 1982, pp. 123-156.

1.18 Seiner, J. M.; McLaughlin, D. K.; and Liu, H.: Supersonic jet noise generated by large-scale instabilities. NASA TP-2072, 1982.

2.1 Ahuja, K. K. ; Lepicovsky, J.; Tam, C. K. W.; Morris, P. J. ; and Burrin, R. H.: Tone-excited jet - theory and experiments. NASA CR-3538, 1982.

2.2 Lush, P. A.; and Burrin, R. H.: The generation and radiation of supersonic jet noise. AFAPL-TR-72-53, Vo1. V - An Experimenta1 Investigation of Jet Noise Variation with Velocity and Temperature, U.S. Air Force, 1972.

2.3 Whiffen, M. C.; and Ahuja, K. K.: An Improved Schlieren System and Some New Results on Acoustica11y Excited Jets. J. Sound Vib., Vo1. 86, 1983, pp. 99-105.

2.4 Goldstein, R. J.: Fluid Mechanics Measurements, (p. 392). Hemisphere Publishing Corporation, 1983.

2.5 Plumblee, H. E.; Burrin, R. H.; Lau, J. C.; Morfry, C. L.; Morris, P.J.; Smith, D. M.; Tanna, H. K.; Tester, B. J.; and Whiffen, M. C.: Laser Velocimeter, Ch. 5 of The Generation and Radiation of Supersonic Jet Noise. AFAPL-TR-76-65, Vo1. II, U. S. Air Force, 1976.

2.6 Lepicovsky, J.; and Bell, W. A.: Aerodynamic measurements about a rotating propeller with a laser velocimeter. J. of Aircraft, Vo1. 21, 1984, pp. 264-271.

2.7 Lepicovsky, J.: Laser Velocimeter measurements of the large-scale structure in a tone-excited jet. AIAA-84-1603, 1984.

2.8 Lepicovsky, J.; Be11, W. A.; and Ahuja, K. K.: Conditional Sampling with a Laser Velocimeter and its Application for Large-Scale Turbulent Structure Measurement. Rep. LG83ER0007, Lockheed-Georgia Company, 1983.

2.9 Bell, W. A.; and Lepicovsky, J.: Conditional Sampling with a Laser Velocimeter. AIAA-83-0756, 1983. 
4.1 Morrison, G. L.; and McLaughlin, D. K.: Instability Process in Low Reynolds Number Supersonic Jets. AIAA Journal, Vol. 18, 1980, pp. 793800 .

4.2 Lau, J. C.; Morris, P. J.; and Fisher, M. J.: Measurement in Subsonic and Supersonic Free Jets Using a Laser Velocimeter. J. Fluid Mech., Vo1. 93, 1979, pp. 1-27.

4.3 Morris, P. J.; and Tam, C. K. W.: Near and Far Noise from Large-Scale Instabilities of Axisymmetric Jets. AIAA Paper, AIAA-77-1351, 1977.

4.4 Stromerg, J. L.; McLaughlin, C. K.; and Troutt, T. R.: Flow Field and Acoustic Properties of a Mach Number 0.9 Jet at a Low Reynolds Number. J. Sound and Vib., Vo1. 72, 1980, pp. 159-176.

4.5 Armstrong, R. R., Jr.: Influence of Mach Number on Coherent Structure Relevant to Jet Noise. AIAA Journal, Vol. 19, 1981, pp. 677-683.

4.6 Lepicovsky, J.; Ahuja, K. K.; and Salikuddin, M.: An Experimental Study of Tone Excited Heated Jets. AIAA Paper, AIAA-84-2341, 1984.

4.7 Lepicovsky, J.; Bell, W. A.; and Ahuja, K. K.: Conditional Sampling with a Laser Velocimeter and its Application for Large-Scale Turbulent Structure Measurement. Lockheed-Georgia Company Report LG83ER0007, 1983.

5.1 Seiner, J. M.; and Norum, T. D.: Aerodynamic Aspects of Shock Containing Jet Plumes, AIAA Paper, AIAA-80-0965, 1980. 


\section{APPENDIX A}

\section{Flow Visualization for Nozzle Selection}

Before the visualization experiments started, an examination of the aerodynamic quality of the available $C-D$ nozzles was made. The flow structures of three separate $C-D$ nozzles were examined. The schlieren pictures of the flow emanating from the first nozzle (1abeled Nozzle A) at three pressure ratios $P_{t} / P_{0}=2.34,3.05$, and 3.39 are shown in Figures $A .1$, $A .2$, and A.3, respectively. It is to be noted that the pressure ratio $\mathrm{P}_{t} / \mathrm{P}_{0}=$ 3.05 provides a fully expanded jet of the design Mach number of $\mathrm{M}_{j}=1.37$. The shock wave systems of the overexpanded jet (Figure A.1) and the underexpanded jet (Figure A. 3) are clearly visible. However, the flow retains a shock structure even for fully-expanded conditions, as seen in Figure A.2. This shows a poor aerodynamic quality of Nozzle A. Closer examination of the nozzle internal surface showed an irregularity of the nozzle contour in the contraction part of the nozzle.

The flow pictures of the second nozzle (labeled Nozzle B) for pressure ratios of $P_{t} / P_{0}=2.49,3.05$, and 3.33 are shown in Figures A.4 through A. 6 . This nozzle also showed a remnant shock wave structure at the fully-expanded design condition (Figure A.4); although in this case, the shocks appear to be weaker than those of Nozzle A.

Finally, the third nozzle (labeled Nozzle c) was found to be acceptable. The schlieren picture of the flow at the fully-expanded condition is shown in Figure A.7. This nozzle, shown in Figure 2.1, was used during the course of the entire experimental work described in this report. 


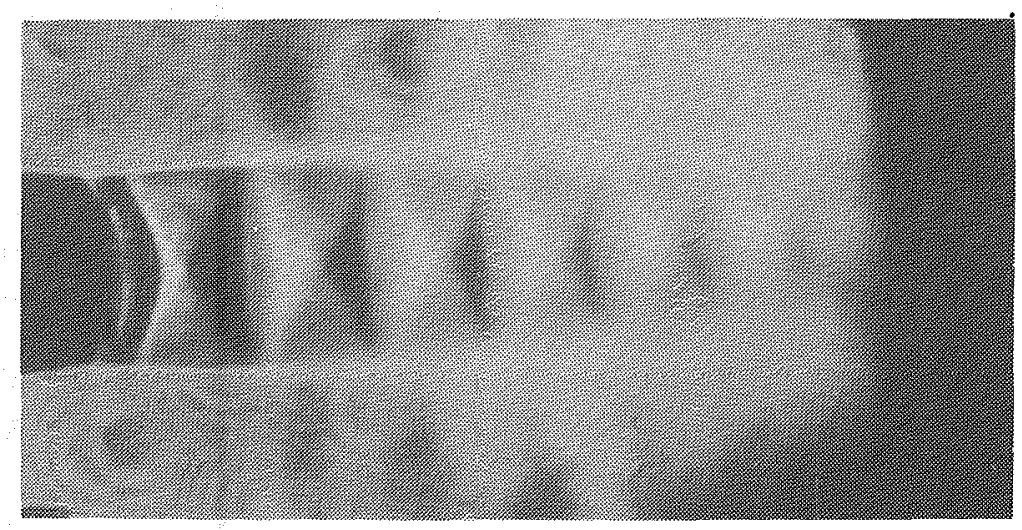

Figure A.1 Nozzle A; overexpanded jet.

(Knife edge: Vertical, $\mathrm{p}_{t} / \mathrm{p}_{\mathrm{o}}=2.34$ )

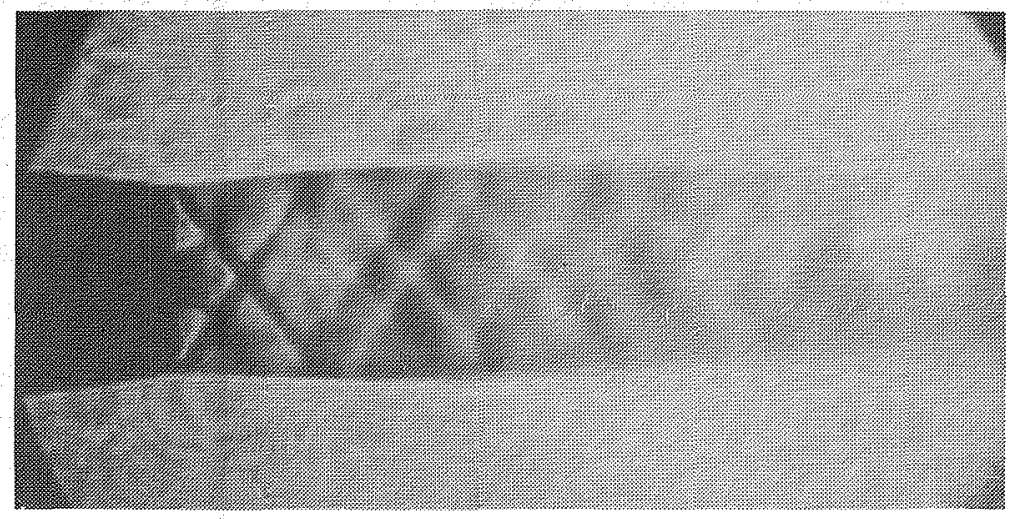

Figure A.2 Nozzle A; fully expanded jet.

(Knife edge: Vertical, $\mathrm{p}_{t} / \mathrm{p}_{\mathrm{o}}=3.05, \mathrm{M}_{j}=1.37$ )

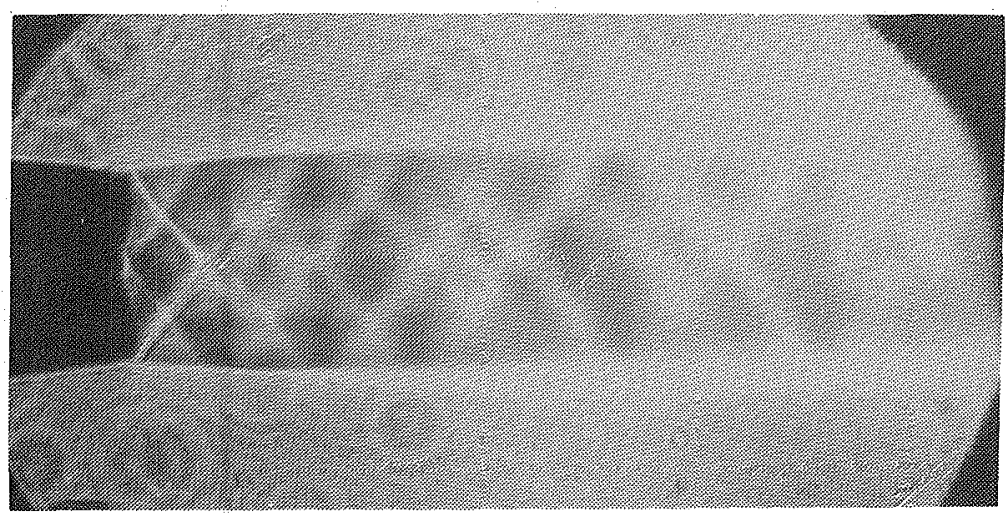

Figure A.3 Nozzle A; underexpanded jet.

(Knife edge: Vertica1, $p_{t} / p_{o}=3.39$ ) 


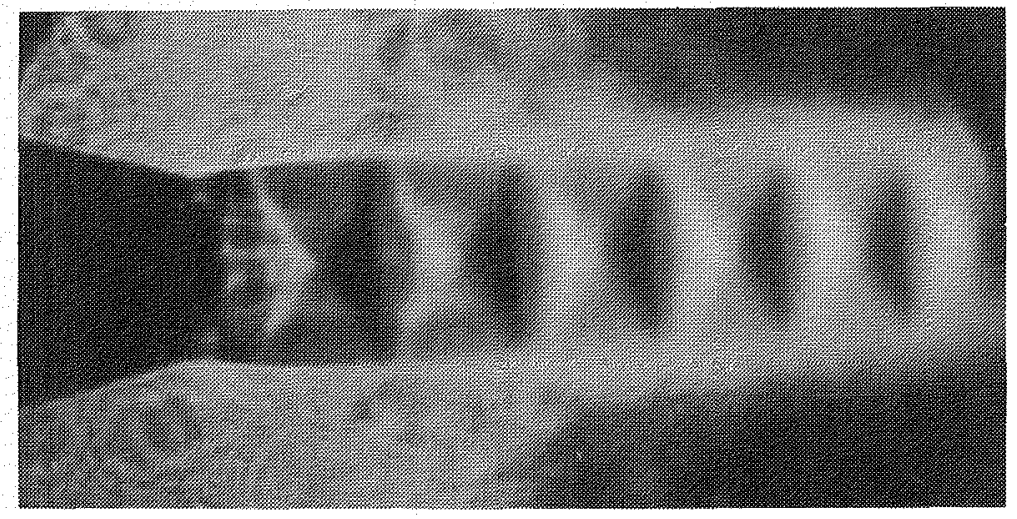

Figure A.4 Nozzle B; overexpanded jet. (Knife edge: Vertical, $\mathrm{p}_{t} / \mathrm{p}_{0}=2.49$ )

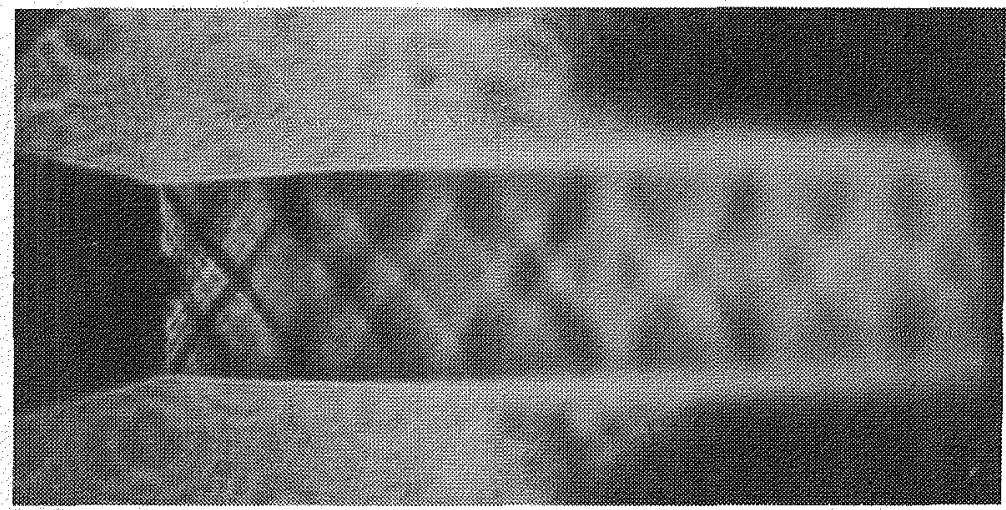

Figure A.5 Nozzle B; fully expanded jet.

(Knife edge: Vertica1, $p_{t} / p_{o}=3.05, M_{j}=1.37$ )

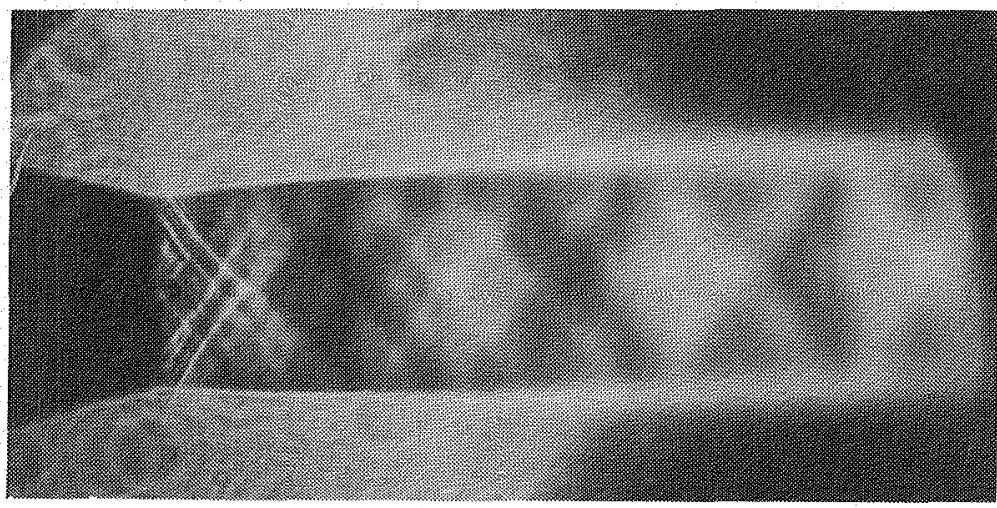

Figure A.6 Nozzle B; underexpanded jet.

(Knife edge: Vertical, $p_{t} / p_{o}=3.33$ ) 


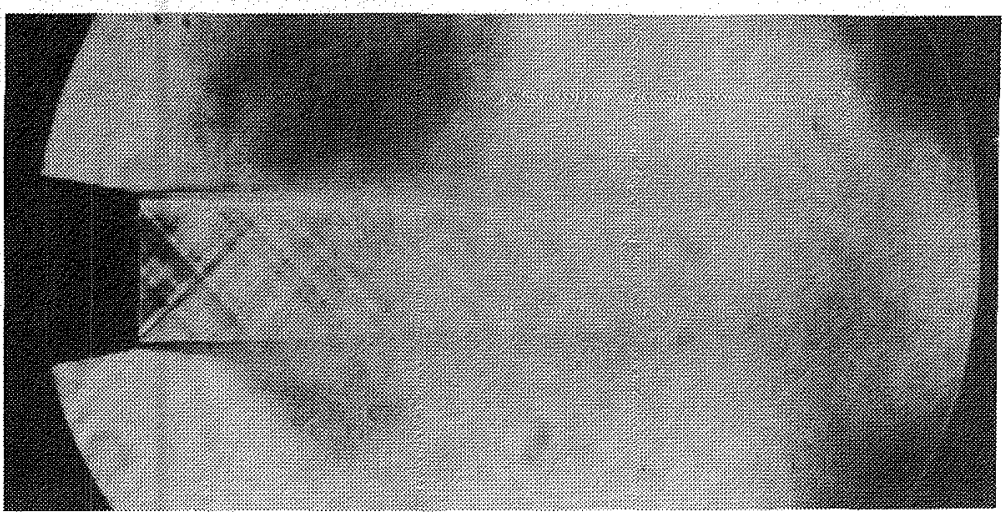

Figure A.7 Nozzle C; fully expanded jet.

(Knife edge: Vertical, $p_{t} / p_{o}=3.05, M_{j}=1.37$ ) 


\section{APPENDIX B}

List of Symbols

a

b

D

f

$i_{u}$

L

M

p

$\mathbf{R}$

S

$T$

U

$\mathrm{v}_{\mathrm{D}}$

$\mathrm{X}, \mathrm{Y}, \mathrm{Z}$

$\phi$

$\xi$

\section{Subscripts:}

c

e

j

s

t

o sound velocity

half shear layer width

nozzle exit diameter

frequency

turbulence intensity (axial component)

sound presgure level measured just outside the nozzle 1ip (rel. $2 \times 10^{-5} \mathrm{~N} / \mathrm{m}^{2}$ )

Mach number

pressure

nozzle exit radius

Strouha1 number

temperature

velocity (axia1 component)

driving voltage

coordinates

phase

nozzle pressure ratio phase velocity

excitation

jet

strobing

tota1

ambient 


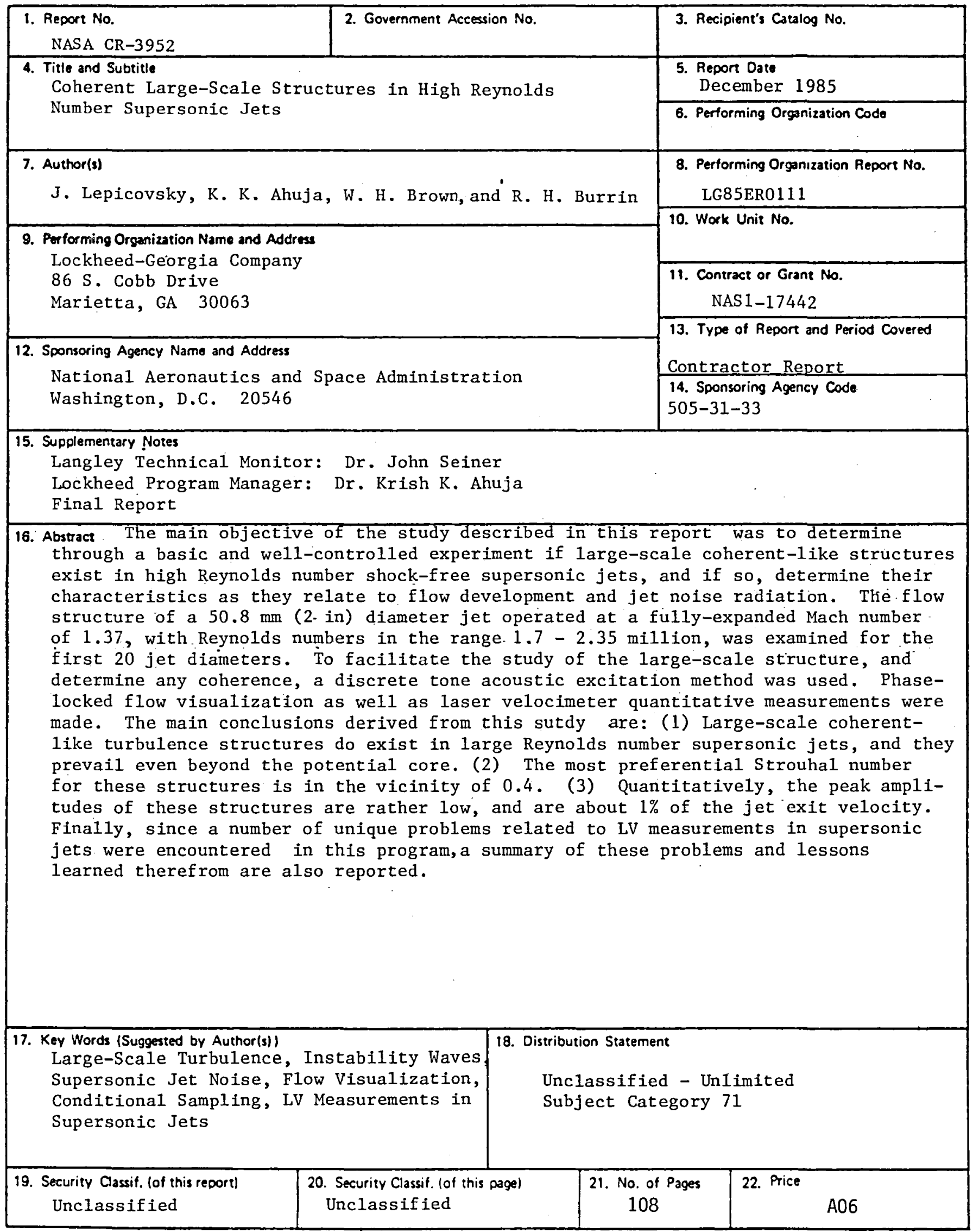

For sale by the National Technical Information Service. Springfield. Virginia 22161 


\section{End of Document}

